\title{
POLYVECTOR FIELDS AND POLYDIFFERENTIAL OPERATORS ASSOCIATED WITH LIE PAIRS
}

\author{
RUGGERO BANDIERA, MATHIEU STIÉNON, AND PING XU
}

\begin{abstract}
We prove that the spaces tot $\left(\Gamma\left(\Lambda^{\bullet} A^{\vee}\right) \otimes_{R} \mathcal{T}_{\text {poly }}^{\bullet}\right)$ and $\operatorname{tot}\left(\Gamma\left(\Lambda^{\bullet} A^{\vee}\right) \otimes_{R} \mathcal{D}_{\text {poly }}^{\bullet}\right)$ associated with a Lie pair $(L, A)$ each carry an $L_{\infty}$ algebra structure canonical up to an $L_{\infty}$ isomorphism with the identity map as linear part. These two spaces serve, respectively, as replacements for the spaces of formal polyvector fields and formal polydifferential operators on the Lie pair $(L, A)$. Consequently, both $\mathbb{H}_{\mathrm{CE}}^{\bullet}\left(A, \mathcal{T}_{\text {poly }}^{\bullet}\right)$ and $\mathbb{H}_{\mathrm{CE}}^{\bullet}\left(A, \mathcal{D}_{\text {poly }}^{\bullet}\right)$ admit unique Gerstenhaber algebra structures. Our approach is based on homotopy transfer and the construction of a Fedosov dg Lie algebroid (i.e. a dg foliation on a Fedosov dg manifold).
\end{abstract}

\section{Contents}

Introduction $\quad 2$

Acknowledgements $\quad 6$

1. Polydifferential operators and polyvector fields for Lie pairs 6

1.1. Chevalley-Eilenberg cohomology 6

1.2. Polydifferential operators $\quad 7$

1.3. Polyvector fields $r$

2. Fedosov dg Lie algebroids 9

2.1. Dg Lie algebroids and polyvector fields and polydifferential operators 9

2.2. Fedosov dg manifolds $\quad 14$

2.3. Fedosov dg Lie algebroids 16

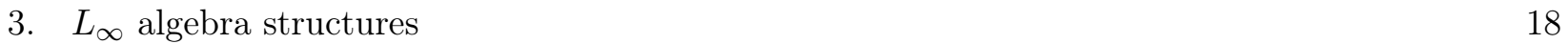

3.1. Dolgushev-Fedosov contraction and $L_{\infty}$ algebra structure on the space of polyvector fields of a Lie pair

3.2. Dolgushev-Fedosov contraction and $L_{\infty}$ algebra structure on the space of polydifferential $\begin{array}{ll}\text { operators of a Lie pair } & 24\end{array}$

3.3. Uniqueness of the $L_{\infty}$ structure 31

4. Matched pair case 33

4.1. Dg Lie algebroid arising from a matched pair 34

4.2. Fedosov dg manifolds associated with matched pairs 35

4.3. Polyvector fields associated with matched pairs 38

4.4. Polydifferential operators associated with matched pairs 40

$\begin{array}{lll}\text { Appendix A. Semifull algebra contractions } & 47\end{array}$

Research partially supported by NSF grants DMS-1406668, DMS-1707545 and DMS-2001599, and NSA grant H9823014-1-0153. 


\section{INTRODUCTION}

The algebraic structures of the spaces of polyvector fields and of polydifferential operators on a manifold play a crucial role in deformation quantization: Kontsevich's famous formality theorem asserts that, for a smooth manifold $M$, the Hochschild-Kostant-Rosenberg map extends to an $L_{\infty}$ quasi-isomorphism from the dgla of polyvector fields on $M$ to the dgla of polydifferential operators on $M[25,46,13,9$, $10]$.

In this paper, we study the algebraic structures of "polyvector fields" and "polydifferential operators" on Lie pairs. Throughout the paper, we use the symbol $\mathbb{k}$ to denote either of the fields $\mathbb{R}$ and $\mathbb{C}$. A Lie algebroid over $\mathbb{k}$ is a $\mathbb{k}$-vector bundle $L \rightarrow M$ together with a bundle map $\varrho: L \rightarrow T_{M} \otimes_{\mathbb{R}} \mathbb{k}$ called anchor and a Lie bracket [-,-] on the sections of $L$ such that $\varrho: \Gamma(L) \rightarrow \mathfrak{X}(M) \otimes_{\mathbb{R}} \mathbb{k}$ is a morphism of Lie algebras and

$$
[X, f Y]=f[X, Y]+\varrho_{X}(f) Y,
$$

for all $X, Y \in \Gamma(L)$ and $f \in C^{\infty}(M, \mathbb{k})$. By a Lie pair $(L, A)$, we mean an inclusion $A \hookrightarrow L$ of Lie algebroids over a smooth manifold $M$.

Lie pairs arise naturally in a number of classical areas of mathematics such as Lie theory, complex geometry, foliation theory, and Poisson geometry. A complex manifold $X$ determines a Lie pair over $\mathbb{C}$ : viz. $L=T_{X} \otimes \mathbb{C}$ and $A=T_{X}^{0,1}$. A foliation $F$ on a smooth manifold $M$ determines a Lie pair over $\mathbb{R}$ : viz. $L=T_{M}$ and $A=T_{F}$ is the integrable distribution on $M$ tangent to the foliation $F$. A manifold equipped with an action of a Lie algebra $\mathfrak{g}$ gives rise to a Lie pair in a natural way (see [39, Example 5.5] and [33, 31]).

Given a Lie pair $(L, A)$, the quotient $L / A$ is naturally an $A$-module [11]. When $L$ is the tangent bundle to a manifold $M$ and $A$ is an integrable distribution on $M$, the infinitesimal $A$-action on $L / A$ is given by the Bott connection [7].

A Lie pair $(L, A)$ gives rise to two natural cochain complexes

$$
\left(\operatorname{tot}\left(\Gamma\left(\Lambda^{\bullet} A^{\vee}\right) \otimes_{R} \mathcal{T}_{\text {poly }}^{\bullet}\right), d_{A}^{\text {Bott }}\right) \quad \text { and } \quad\left(\operatorname{tot}\left(\Gamma\left(\Lambda^{\bullet} A^{\vee}\right) \otimes_{R} \mathcal{D}_{\text {poly }}^{\bullet}\right), d_{A}^{\mathcal{U}}+\mathfrak{d}_{\mathscr{H}}\right)
$$

constructed as follows. Denoting the algebra of smooth functions on the manifold $M$ by $R$, we set $\mathcal{T}_{\text {poly }}^{\bullet}=\bigoplus_{k=-1}^{\infty} \mathcal{T}_{\text {poly }}^{k}$, where $\mathcal{T}_{\text {poly }}^{-1}=R$ and $\mathcal{T}_{\text {poly }}^{k}=\Gamma\left(\Lambda^{k+1}(L / A)\right)$ for $k \geqslant 0$. The Bott $A$-connection on $L / A$ makes every $\mathcal{T}_{\text {poly }}^{k}$ an $A$-module. We can thus consider the complex of $A$-modules with trivial differential

$$
0 \longrightarrow \mathcal{T}_{\text {poly }}^{-1} \stackrel{0}{\longrightarrow} \mathcal{T}_{\text {poly }}^{0} \stackrel{0}{\longrightarrow} \mathcal{T}_{\text {poly }}^{1} \stackrel{0}{\longrightarrow} \mathcal{T}_{\text {poly }}^{2} \stackrel{0}{\longrightarrow} \cdots
$$

Its Chevalley-Eilenberg cohomology $\mathbb{H}_{\mathrm{CE}}^{\bullet}\left(A, \mathcal{T}_{\text {poly }}^{\bullet}\right)$ is the cohomology of the total cochain complex

$$
\left(\operatorname{tot}\left(\Gamma\left(\Lambda^{\bullet} A^{\vee}\right) \otimes_{R} \mathcal{T}_{\text {poly }}^{\bullet}\right), d_{A}^{\text {Bott }}\right) .
$$

Similarly, denoting the universal enveloping algebra of the Lie algebroid $L$ by $\mathcal{U}(L)$, we set $\mathcal{D}_{\text {poly }}^{\bullet}=\bigoplus_{k=-1}^{\infty} \mathcal{D}_{\text {poly }}^{k}$, where $\mathcal{D}_{\text {poly }}^{-1}=R ; \mathcal{D}_{\text {poly }}^{0}=\frac{\mathcal{U}(L)}{\mathcal{U}(L) \Gamma(A)}$; and $\mathcal{D}_{\text {poly }}^{k}$ with $k \geqslant 1$ is the tensor product $\mathcal{D}_{\text {poly }}^{0} \otimes_{R} \cdots \otimes_{R} \mathcal{D}_{\text {poly }}^{0}$ of $(k+1)$-copies of the left $R$-module $\mathcal{D}_{\text {poly }}^{0}$. Multiplication in $\mathcal{U}(L)$ from the left by elements of $\Gamma(A)$ (and $R$ ) induces an $A$-module structure on the quotient $\frac{\mathcal{U}(L)}{\mathcal{U}(L) \Gamma(A)}$. This action of $A$ on $\mathcal{D}_{\text {poly }}^{0}$ extends naturally to an action of $A$ on $\mathcal{D}_{\text {poly }}^{k}$ for each $k \geqslant 1$. In fact, $\mathcal{D}_{\text {poly }}^{0}$ is a 
cocommutative coassociative coalgebra over $R$ whose comultiplication $\Delta: \mathcal{D}_{\text {poly }}^{0} \rightarrow \mathcal{D}_{\text {poly }}^{0} \otimes_{R} \mathcal{D}_{\text {poly }}^{0}$ is a morphism of $A$-modules. Therefore, the induced Hochschild complex

$$
0 \longrightarrow \mathcal{D}_{\text {poly }}^{-1} \stackrel{d_{\mathscr{H}}}{\longrightarrow} \mathcal{D}_{\text {poly }}^{0} \stackrel{d_{\mathscr{H}}}{\longrightarrow} \mathcal{D}_{\text {poly }}^{1} \stackrel{d_{\mathscr{H}}}{\longrightarrow} \mathcal{D}_{\text {poly }}^{2} \stackrel{d_{\mathscr{H}}}{\longrightarrow} \cdots
$$

is a complex of $A$-modules. Its Chevalley-Eilenberg cohomology $\mathbb{H}_{\mathrm{CE}}^{\bullet}\left(A, \mathcal{D}_{\text {poly }}^{\bullet}\right)$ is the cohomology of the total cochain complex

$$
\left(\operatorname{tot}\left(\Gamma\left(\Lambda^{\bullet} A^{\vee}\right) \otimes_{R} \mathcal{D}_{\text {poly }}^{\bullet}\right), d_{A}^{\mathcal{U}}+\mathfrak{d}_{\mathscr{H}}\right),
$$

where we use the abbreviated symbol $\mathfrak{d}_{\mathscr{H}}$ to denote the operator id $\otimes d_{\mathscr{H}}$ - see Equation (7) for more details.

For instance, for the Lie pair $L=T_{X} \otimes \mathbb{C}$ and $A=T_{X}^{0,1}$ arising from any complex manifold $X$, the cochain complexes (2) and (3) are precisely the complexes $\left(\Omega^{0, \bullet}\left(\mathcal{T}_{\text {poly }}^{\bullet}(X)\right), \bar{\partial}\right)$ and $\left(\Omega^{0, \bullet}\left(\mathcal{D}_{\text {poly }}^{\bullet}(X)\right), \bar{\partial}+d_{\mathscr{H}}\right)$, which are known to carry differential graded Lie algebra (a.k.a. dgla) structures. The corresponding Chevalley-Eilenberg cohomology groups $\mathbb{H}_{\mathrm{CE}}^{\bullet}\left(A, \mathcal{T}_{\text {poly }}^{\bullet}\right)$ and $\mathbb{H}_{\mathrm{CE}}^{\bullet}\left(A, \mathcal{D}_{\text {poly }}^{\bullet}\right)$ are isomorphic to the sheaf cohomology group $\mathbb{H}^{\bullet}\left(X, \Lambda^{\bullet} T_{X}\right)$ and the Hochschild cohomology group $H H^{\bullet}(X)$, respectively.

For a generic Lie pair $(L, A)$, however, there is no obvious way to upgrade the cochain complexes $(1)$ to dgla's (or $L_{\infty}$ algebras). Here is an example. The cochain complex $\left(\operatorname{tot} \Omega_{F}^{\bullet}\left(\Lambda^{\bullet}\left(T_{M} / T_{F}\right)\right), d_{F}^{\text {Bott }}\right)$ associated with the Lie pair $\left(T_{M}, T_{F}\right)$ encoding a foliation $F$ on a smooth manifold $M$ may be thought of as the space of formal polyvector fields on the leaf space of the foliation [49, 50], or more precisely, on the differentiable stack [4] presented by the holonomy groupoid of the foliation $F$. Similarly, denoting the associative algebra of differential operators on $M$ by $\mathfrak{D}(M)$, the cochain complex $\left(\right.$ tot $\left.\Omega_{F}^{\bullet}\left(\bigotimes_{R}^{\bullet}\left(\frac{\mathfrak{D}(M)}{\mathfrak{D}(M) \cdot \Gamma\left(T_{F}\right)}\right)\right), d_{A}^{\mathcal{U}}+\mathfrak{d}_{\mathscr{H}}\right)$ may be thought of as the space of formal polydifferential operators on the leaf space of the foliation, or more precisely, on the differentiable stack presented by the holonomy groupoid of the foliation $F$. Unless the foliation $F$ admits a transversal foliation [12], there are no obvious dgla (or $L_{\infty}$ algebra) structures on these cochain complexes.

On the other hand, both $\mathbb{H}_{\mathrm{CE}}^{\bullet}\left(A, \mathcal{T}_{\text {poly }}^{\bullet}\right)$ and $\mathbb{H}_{\mathrm{CE}}^{\bullet}\left(A, \mathcal{D}_{\text {poly }}^{\bullet}\right)$ admit obvious associative algebra structures - the multiplications in cohomology proceed from the wedge product in $\mathcal{T}_{\text {poly }}^{\bullet}$ and the tensor product of left $R$-modules in $\mathcal{D}_{\text {poly }}^{\bullet}$

We are thus naturally led to the following central twofold question:

\section{Question.}

(1) Do the cohomology groups $\mathbb{H}_{\mathrm{CE}}^{\bullet}\left(A, \mathcal{T}_{\text {poly }}^{\bullet}\right)$ and $\mathbb{H}_{\mathrm{CE}}^{\bullet}\left(A, \mathcal{D}_{\text {poly }}^{\bullet}\right)$ admit canonical Gerstenhaber algebra structures?

(2) Do the two cochain complexes

$$
\left(\operatorname{tot}\left(\Gamma\left(\Lambda^{\bullet} A^{\vee}\right) \otimes_{R} \mathcal{T}_{\text {poly }}^{\bullet}\right), d_{A}^{\text {Bott }}\right) \quad \text { and } \quad\left(\operatorname{tot}\left(\Gamma\left(\Lambda^{\bullet} A^{\vee}\right) \otimes_{R} \mathcal{D}_{\text {poly }}^{\bullet}\right), d_{A}^{\mathcal{U}}+\mathfrak{d}_{\mathscr{H}}\right)
$$

associated with a Lie pair $(L, A)$ admit $L_{\infty}$ algebra structures compatible "in a certain sense" with their respective associative multiplications? If so, are these $L_{\infty}$ structures canonical?

To answer this question, we introduce the notion of Fedosov dg Lie algebroid, we establish a pair of contractions, and we apply the homotopy transfer theorem of $L_{\infty}$ algebras $[5,22,17]$ (see also $[1,16$, $6,14,15])$. Roughly speaking, given a Lie pair $(L, A)$, we construct a geometric object called Fedosov dg Lie algebroid, which engenders a pair of natural dgla's whose respective cohomologies carry natural Gerstenhaber algebra structures. The pair of cochain complexes underlying these engendered dgla's are homotopy equivalent (in a style reminiscent of Dolgushev's Fedosov resolutions [13]) to the cochain complexes (1) associated with the Lie pair $(L, A)$. The latter complexes then inherit $L_{\infty}$ structures by homotopy transfer. 
Hereunder, we proceed to give a more detailed outline of the construction.

Given a Lie pair $(L, A)$ and having chosen some additional geometric data, one can endow the graded manifold $\mathcal{M}=L[1] \oplus L / A$ with a homological vector field $Q$ encoding the formal geometry of the Lie pair. The resulting dg manifold $(\mathcal{M}, Q)$ is called a Fedosov dg manifold [45]. It turns out that there exists a natural $\mathrm{dg}$ integrable distribution $\mathcal{F} \subset T_{\mathcal{M}}$ on $(\mathcal{M}, Q)$. In other words, the tangent dg Lie algebroid $T_{\mathcal{M}} \rightarrow \mathcal{M}$ arising from the Fedosov dg manifold $(\mathcal{M}, Q)$ admits a natural dg Lie subalgebroid $\mathcal{F} \rightarrow \mathcal{M}$. We call this dg Lie algebroid $\mathcal{F} \rightarrow \mathcal{M}$ a Fedosov dg Lie algebroid.

Lie algebroids being generalizations of tangent bundles, the notions of polyvector fields and of polydifferential operators admit generalizations to the broader context of Lie algebroids. The spaces of (generalized) polyvectors fields and of (generalized) polydifferential operators each admit a natural dgla structure and the cohomology of this dgla is in fact a Gerstenhaber algebra [54, 55]. The notions of polyvector fields and of polydifferential operators can be extended further in an appropriate sense to the context of $d g$ Lie algebroids. This yields again a pair of dgla's whose cohomologies are Gerstenhaber algebras.

More precisely, in the context of a dg Lie algebroid $\mathcal{L} \rightarrow \mathcal{M}$, a $k$-vector field is a section of the vector bundle $\Lambda^{k} \mathcal{L} \rightarrow \mathcal{M}$ while a $k$-differential operator is an element of $(s \mathcal{U}(\mathcal{L}))^{\otimes k}$, the tensor product (as left $C^{\infty}(\mathcal{M})$-modules) of $k$ copies of the suspended universal enveloping algebra $s \mathcal{U}(\mathcal{L})$.

It is clear that the differential $\mathcal{Q}: \Gamma(\mathcal{L}) \rightarrow \Gamma(\mathcal{L})$, the homological vector field $Q: C^{\infty}(\mathcal{M}) \rightarrow C^{\infty}(\mathcal{M})$, and the Lie bracket on $\Gamma(\mathcal{L})$ encoding the dg Lie algebroid structure of $\mathcal{L} \rightarrow \mathcal{M}$ extend naturally to a degree $(+1)$ differential $\mathcal{Q}: \Gamma\left(\Lambda^{k+1} \mathcal{L}\right) \rightarrow \Gamma\left(\Lambda^{k+1} \mathcal{L}\right)$ and a Schouten bracket $[-,-]: \Gamma\left(\Lambda^{u+1} \mathcal{L}\right) \otimes \Gamma\left(\Lambda^{v+1} \mathcal{L}\right) \rightarrow \Gamma\left(\Lambda^{u+v+1} \mathcal{L}\right)$ - see Section 2.1 for more details. The resulting triple $\left(\operatorname{tot}_{\oplus} \Gamma\left(\Lambda^{\bullet+1} \mathcal{L}\right), \mathcal{Q},[-,-]\right)$ is a dgla.

The universal enveloping algebra of a $d g$ Lie algebroid $\mathcal{L} \rightarrow \mathcal{M}$, which is defined by adapting the construction of the universal enveloping algebra of a Lie algebroid, is a $\mathrm{dg}$ Hopf algebroid $\mathcal{U}(\mathcal{L})$ over the dgca $R=C^{\infty}(\mathcal{M})$. For each $k \geqslant 0$, the dg structure on the dg Lie algebroid $\mathcal{L} \rightarrow \mathcal{M}$ determines a differential $\mathcal{Q}:(s \mathcal{U}(\mathcal{L}))^{\otimes k+1} \rightarrow(s \mathcal{U}(\mathcal{L}))^{\otimes k+1}$ of degree $(+1)$. A Hochschild coboundary differential $d_{\mathscr{H}}:(s \mathcal{U}(\mathcal{L}))^{\otimes k} \rightarrow(s \mathcal{U}(\mathcal{L}))^{\otimes k+1}$ and a Gerstenhaber bracket $\llbracket-,-\rrbracket:(s \mathcal{U}(\mathcal{L}))^{\otimes u+1} \otimes(s \mathcal{U}(\mathcal{L}))^{\otimes v+1} \rightarrow(s \mathcal{U}(\mathcal{L}))^{\otimes u+v}$ can be defined explicitly in terms of the dg Hopf algebroid structure. The resulting triple $\left(\operatorname{tot}_{\oplus}(s \mathcal{U}(\mathcal{L}))^{\otimes \bullet+1}, \mathcal{Q}+d_{\mathscr{H}}, \llbracket-,-\rrbracket\right)$ is a dgla.

The "polyvector fields" and "polydifferential operators" associated with a Fedosov dg Lie algebroid $\mathcal{F} \rightarrow \mathcal{M}$ may be viewed geometrically as polyvector fields and polydifferential operators tangent to the $\mathrm{dg}$ foliation $\mathcal{F}$ on the Fedosov dg manifold $(\mathcal{M}, Q)$. In fact, one can identify the "polyvector fields" $\left(\operatorname{tot}_{\oplus} \Gamma\left(\Lambda^{\bullet+1} \mathcal{F}\right), \mathcal{Q}\right)$ and "polydifferential operators" $\left(\operatorname{tot}_{\oplus}(s \mathcal{U}(\mathcal{F}))^{\otimes \bullet+1}, \mathcal{Q}+d_{\mathscr{H}}\right)$ associated with $\mathcal{F} \rightarrow \mathcal{M}$ to a pair of cochain complexes

$$
\left(\operatorname{tot}\left(\Gamma\left(\Lambda^{\bullet} L^{\vee}\right) \otimes_{R} \mathscr{T}_{\text {poly }}^{\bullet}\right), \mathcal{L}_{Q}\right) \quad \text { and } \quad\left(\operatorname{tot}\left(\Gamma\left(\Lambda^{\bullet} L^{\vee}\right) \otimes_{R} \mathscr{D}_{\text {poly }}^{\bullet}\right), \llbracket Q+m,-\rrbracket\right),
$$

where $\mathscr{T}_{\text {poly }}^{\bullet}$ denotes the formal polyvector fields and $\mathscr{D}_{\text {poly }}^{\bullet}$ the formal polydifferential operators tangent to the fibers of the vector bundle $L / A \rightarrow M$.

The next step and key ingredient of the construction consists in establishing the following pair of contractions of Dolgushev-Fedosov type:

$$
\left(\operatorname{tot}\left(\Gamma\left(\Lambda^{\bullet} A^{\vee}\right) \otimes_{R} \mathcal{T}_{\text {poly }}^{\bullet}\right), d_{A}^{\text {Bott }}\right) \rightleftarrows\left(\operatorname{tot}\left(\Gamma\left(\Lambda^{\bullet} L^{\vee}\right) \otimes_{R} \mathscr{T}_{\text {poly }}^{\bullet}\right), \mathcal{L}_{Q}\right) \supset
$$

and

$$
\left(\operatorname{tot}\left(\Gamma\left(\Lambda^{\bullet} A^{\vee}\right) \otimes_{R} \mathcal{D}_{\text {poly }}^{\bullet}\right), d_{A}^{\mathcal{U}}+\mathfrak{d}_{\mathscr{H}}\right) \rightleftarrows\left(\operatorname{tot}\left(\Gamma\left(\Lambda^{\bullet} L^{\vee}\right) \otimes_{R} \mathscr{D}_{\text {poly }}^{\bullet}\right), \llbracket Q+m,-\rrbracket\right) \supset
$$


Finally, we use the homotopy transfer theorem for $L_{\infty}$ algebras $[5,22,17]$ - see also $[1,16,6,14,15]$ — to push the $L_{\infty}$ structures carried by the complexes (4) (the r.h.s. of the contractions (5) and (6)) to the complexes (1) (the l.h.s. of the contractions (5) and (6)). Furthermore, we prove that the resulting $L_{\infty}$ algebra structures on the complexes (1) are unique up to $L_{\infty}$ isomorphisms having the identity map as linear part and are therefore (essentially) independent of the choice of geometric data made in the construction of the Fedosov dg Lie algebroid. Moreover, we prove that the two cochain maps in the above contractions (5) and (6) are compatible with the associative algebra structures given by the wedge and cup products respectively.

Finally, combining these facts, we are able to prove the following theorem, which is the main result of the paper.

Theorem A. Let $(L, A)$ be a Lie pair.

(1) The cohomology groups $\mathbb{H}_{\mathrm{CE}}^{\bullet}\left(A, \mathcal{T}_{\text {poly }}^{\bullet}\right)$ and $\mathbb{H}_{\mathrm{CE}}^{\bullet}\left(A, \mathcal{D}_{\text {poly }}^{\bullet}\right)$ admit canonical Gerstenhaber algebra structures.

(2a) The spaces tot $\left(\Gamma\left(\Lambda^{\bullet} A^{\vee}\right) \otimes_{R} \mathcal{T}_{\text {poly }}^{\bullet}\right)$ and tot $\left(\Gamma\left(\Lambda^{\bullet} A^{\vee}\right) \otimes_{R} \mathcal{D}_{\text {poly }}^{\bullet}\right)$ admit $L_{\infty}$ algebra structures with the operators $d_{A}^{\mathrm{Bott}}$ and $d_{A}^{\mathcal{U}}+\mathfrak{d} \mathscr{H}$ as their respective unary brackets.

(2b) These $L_{\infty}$ algebra structures are unique up to $L_{\infty}$ isomorphisms having the identity map as linear part.

(2c) The binary brackets are compatible with the associative products (viz. the wedge product and the cup product respectively) in the sense that the graded Leibniz rule holds up to homotopy.

The above theorem is a synthesis of Propositions 3.9, 3.24 and 3.26 from this paper. We remark that, in Theorem A (2c), we only claim what is needed to ensure that the resulting cohomology groups are Gerstenhaber algebras, but in fact the $L_{\infty}$ and associative algebra structures should be compatible in a much stronger and refined sense. More precisely, the space of polyvector fields tot $\left(\Gamma\left(\Lambda^{\bullet} A^{\vee}\right) \otimes_{R} \mathcal{T}_{\text {poly }}^{\bullet}\right)$ and that of polydifferential operators tot $\left(\Gamma\left(\Lambda^{\bullet} A^{\vee}\right) \otimes_{R} \mathcal{D}_{\text {poly }}^{\bullet}\right)$ should both carry much richer algebraic structures, such as the $\mathrm{Ger}_{\infty}$ algebras investigated by Tamarkin [46] or the $\mathrm{Br}_{\infty}$ algebras studied by Willwacher [53]. In fact, it should again be possible to construct such structures explicitly via homotopy transfer along Dolgushev-Fedosov contractions. We will return to this issue in a forthcoming work.

When the Lie algebroid $L$ arises as the matched sum $A \bowtie B$ of a matched pair $(A, B)$ of Lie algebroids, i.e. when the short exact sequence $0 \rightarrow A \rightarrow L \rightarrow L / A \rightarrow 0$ admits a splitting $j: L / A \rightarrow L$ whose image $B:=j(L / A)$ is a Lie subalgebroid of $L$, the $L_{\infty}$ algebra structures on tot $\left(\Gamma\left(\Lambda^{\bullet} A^{\vee}\right) \otimes_{R} \mathcal{T}_{\text {poly }}^{\bullet}\right)$ and tot $\left(\Gamma\left(\Lambda^{\bullet} A^{\vee}\right) \otimes_{R} \mathcal{D}_{\text {poly }}^{\bullet}\right)$ in Theorem A turn out to be dgla's and admit a much simpler description than in the case of a generic Lie pair. Indeed, in the case of a matched pair, the dg manifold $\left(A[1] \oplus B, d_{A}^{\text {Bott }}\right)$ is a dg Lie algebroid over the $\mathrm{dg}$ manifold $\left(A[1], d_{A}\right)$ whose associated cochain complexes of polyvector fields and polydifferential operators are isomorphic to $\left(\operatorname{tot} \Gamma\left(\Lambda^{\bullet} A^{\vee} \otimes \Lambda^{\bullet+1} B\right), d_{A}^{\text {Bott }}\right)$ and $\left(\operatorname{tot}\left(\Gamma\left(\Lambda^{\bullet} A^{\vee}\right) \otimes_{R} \mathcal{U}(B)^{\otimes \bullet+1}\right), d_{A}^{\mathcal{U}}+\mathfrak{d} \mathscr{H}\right)$, respectively, and are therefore naturally dgla's when endowed with the usual Schouten bracket and the usual Gerstenhaber bracket, respectively.

Theorem B. If, in a Lie pair $(L, A)$, the Lie algebroid $L$ arises as the matched sum $A \bowtie B$ of a matched pair $(A, B)$ of Lie algebroids - i.e. the short exact sequence $0 \rightarrow A \rightarrow L \rightarrow L / A \rightarrow 0$ admits a splitting $j: L / A \rightarrow L$ whose image $B:=j(L / A)$ is a Lie subalgebroid of $L-$ then the $L_{\infty}$ algebra structures of Theorem $A$ on

$$
\operatorname{tot}\left(\Gamma\left(\Lambda^{\bullet} A^{\vee}\right) \otimes_{R} \mathcal{T}_{\text {poly }}^{\bullet}\right) \quad \text { and } \quad \operatorname{tot}\left(\Gamma\left(\Lambda^{\bullet} A^{\vee}\right) \otimes_{R} \mathcal{D}_{\text {poly }}^{\bullet}\right)
$$

are actually dgla's and are respectively isomorphic to

$$
\left(\operatorname{tot} \Gamma\left(\Lambda^{\bullet} A^{\vee} \otimes \Lambda^{\bullet+1} B\right), d_{A}^{\text {Bott }},[-,-]\right) \quad \text { and }\left(\operatorname{tot}\left(\Gamma\left(\Lambda^{\bullet} A^{\vee}\right) \otimes_{R} \mathcal{U}(B)^{\otimes \bullet+1}\right), d_{A}^{\mathcal{U}}+\mathfrak{d}_{\mathscr{H}}, \llbracket-,-\rrbracket\right) \text {, }
$$


the dgla's of polyvector fields and of polydifferential operators arising from the dg Lie algebroid $A[1] \oplus B \rightarrow A[1]$. The isomorphisms are canonical. Furthermore, the Gerstenhaber algebra structures on the corresponding cohomology groups

$$
\mathbb{H}_{\mathrm{CE}}^{\bullet}\left(A, \mathcal{T}_{\text {poly }}^{\bullet}\right) \quad \text { and } \quad \mathbb{H}_{\mathrm{CE}}^{\bullet}\left(A, \mathcal{D}_{\text {poly }}^{\bullet}\right)
$$

are isomorphic to the canonical Gerstenhaber algebra structures on

$$
\mathbb{H}_{\mathrm{CE}}^{\bullet}\left(A, \Lambda^{\bullet+1} B\right) \quad \text { and } \quad \mathbb{H}_{\mathrm{CE}}^{\bullet}\left(A, \mathcal{U}(B)^{\otimes \bullet+1}\right),
$$

respectively.

Finally, let us recall that the well-known Hochschild-Kostant-Rosenberg map for ordinary smooth manifolds admits a natural generalization as a morphism from tot $\left(\Gamma\left(\Lambda^{\bullet} A^{\vee}\right) \otimes_{R} \mathcal{T}_{\text {poly }}^{\bullet}\right)$ to tot $\left(\Gamma\left(\Lambda^{\bullet} A^{\vee}\right) \otimes_{R} \mathcal{D}_{\text {poly }}^{\bullet}\right)$, which is still a quasi-isomorphism of cochain complexes and thus induces, on the cohomology level, an isomorphism from $\mathbb{H}_{\mathrm{CE}}^{\bullet}\left(A, \mathcal{T}_{\text {poly }}^{\bullet}\right)$ to $\mathbb{H}_{\mathrm{CE}}^{\bullet}\left(A, \mathcal{D}_{\text {poly }}^{\bullet}\right)$. However, there is a significant difference compared to the case of ordinary smooth manifolds: the Hochschild-KostantRosenberg map for Lie pairs does not in general respect the Gerstenhaber algebra structures on cohomology. Nervertheless, it is always possible to remedy this defect: the Hochschild-KostantRosenberg morphism must be twisted. Doing so involves techniques developed by Kontsevich in the proof of his formality theorem [25] — see also [46]. Indeed, the present paper provides the foundation for an ulterior paper [30] establishing a formality theorem for Lie pairs and an ensuing Kontsevich-Duflo type theorem describing the precise relationship between the Gerstenhaber algebra structures on $\mathbb{H}_{\mathrm{CE}}^{\bullet}\left(A, \mathcal{T}_{\text {poly }}^{\bullet}\right)$ and $\mathbb{H}_{\mathrm{CE}}^{\bullet}\left(A, \mathcal{D}_{\text {poly }}^{\bullet}\right)$ revealed in Theorem $\mathrm{A}$.

Acknowledgements. We would like to thank Martin Bordemann, Damien Broka, Zhuo Chen, Olivier Elchinger, Vasiliy Dolgushev, Camille Laurent-Gengoux, Hsuan-Yi Liao, Kirill Mackenzie, Rajan Mehta, Jim Stasheff, Luca Vitagliano, and Yannick Voglaire for fruitful discussions and useful comments. We are grateful to an anonymous referee for many insightful comments and suggestions which led to sensible improvements in the presentation of our results. Stiénon is grateful to Universite Paris 7 for its hospitality during his sabbatical leave in 2015-2016.

\section{Polydifferential operators AND POlyVector Fields For Lie Pairs}

1.1. Chevalley-Eilenberg cohomology. Let $A \rightarrow M$ be a Lie algebroid. The Chevalley-Eilenberg cohomology $\mathbb{H}_{\mathrm{CE}}^{k}\left(A, \mathcal{E}^{\bullet}\right)$ in degree $k$ of a complex of left $\mathcal{U}(A)$-modules

$$
0 \longrightarrow \mathcal{E}^{-1} \stackrel{d}{\longrightarrow} \mathcal{E}^{0} \stackrel{d}{\longrightarrow} \mathcal{E}^{1} \stackrel{d}{\longrightarrow} \mathcal{E}^{2} \stackrel{d}{\longrightarrow} \cdots
$$

is the total cohomology in degree $k$ of the double complex

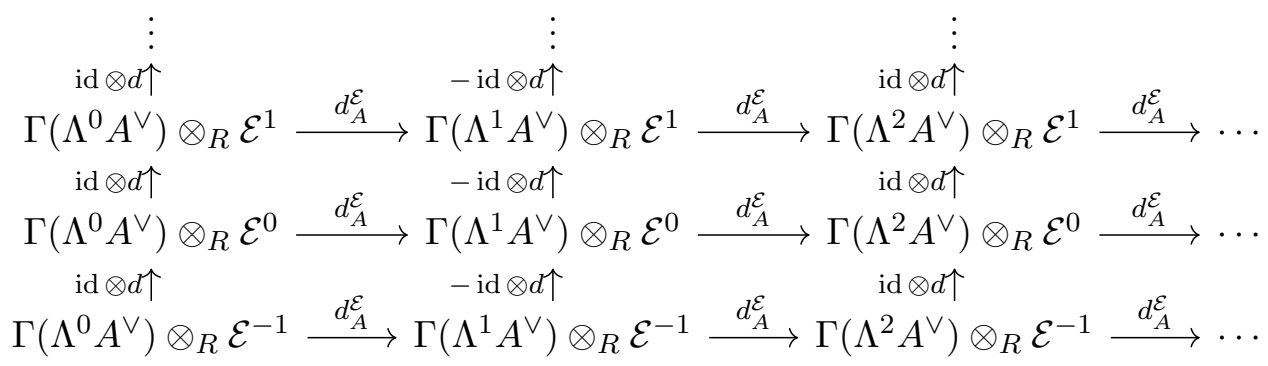

When we say that the above diagram is a double complex, we mean in particular that each square of the grid commutes. Hence the total cohomology is the cohomology of the complex

$$
\left(\bigoplus_{p+q=\bullet} \Gamma\left(\Lambda^{p} A^{\vee}\right) \otimes_{R} \mathcal{E}^{q}, d_{A}^{\mathcal{E}}+\mathrm{id} \otimes d\right) \text {. }
$$


Recall that, the degree of the operator $d$ being +1 , the usual sign convention for the tensor product of linear maps in the presence of gradings dictates that

$$
(\mathrm{id} \otimes d)(\omega \otimes e)=(-1)^{p} \omega \otimes d(e), \quad \forall \omega \in \Gamma\left(\Lambda^{p} A^{\vee}\right), \forall e \in \mathcal{E}^{\bullet} .
$$

1.2. Polydifferential operators. Given a Lie pair $(L, A)$, let $\mathcal{D}_{\text {poly }}^{-1}$ denote the algebra $R$ of smooth functions on the manifold $M$, let $\mathcal{D}_{\text {poly }}^{0}$ denote the left $\mathcal{U}(A)$-module $\frac{\mathcal{U}(L)}{\mathcal{U}(L) \Gamma(A)}$, let $\mathcal{D}_{\text {poly }}^{k}$ denote the tensor product $\mathcal{D}_{\text {poly }}^{0} \otimes_{R} \cdots \otimes_{R} \mathcal{D}_{\text {poly }}^{0}$ of $(k+1)$ copies of the left $R$-module $\mathcal{D}_{\text {poly }}^{0}$, and set $\mathcal{D}_{\text {poly }}^{\bullet}=\bigoplus_{k=-1}^{\infty} \mathcal{D}_{\text {poly }}^{k}$. Since $\mathcal{D}_{\text {poly }}^{0}$ is a left $\mathcal{U}(A)$-module and $\mathcal{U}(A)$, as a Hopf algebroid, is endowed with a comultiplication, $\mathcal{D}_{\text {poly }}^{k}$ is also naturally a left $\mathcal{U}(A)$-module for each $k \geqslant-1$ [55].

Furthermore, the comultiplication $\Delta: \mathcal{U}(L) \rightarrow \mathcal{U}(L) \otimes_{R} \mathcal{U}(L)$ on the universal enveloping algebra $\mathcal{U}(L)$ induces a comultiplication

$$
\Delta: \mathcal{D}_{\text {poly }}^{0} \rightarrow \mathcal{D}_{\text {poly }}^{0} \otimes_{R} \mathcal{D}_{\text {poly }}^{0}
$$

since

$$
\Delta(\mathcal{U}(L) \Gamma(A)) \subseteq \mathcal{U}(L) \otimes_{R}(\mathcal{U}(L) \Gamma(A))+(\mathcal{U}(L) \Gamma(A)) \otimes_{R} \mathcal{U}(L)
$$

- see [29, Sections 2.2 and 2.3].

Lemma $1.1([29])$. The $\mathcal{U}(A)$-module $\mathcal{D}_{\text {poly }}^{0}$ is a cocommutative coassociative coalgebra over $R$ whose comultiplication $\Delta: \mathcal{D}_{\text {poly }}^{0} \rightarrow \mathcal{D}_{\text {poly }}^{0} \otimes_{R} \mathcal{D}_{\text {poly }}^{0}$ is a morphism of $\mathcal{U}(A)$-modules.

Following [55, Equation (98)], introduce the Hochschild differential $d_{\mathscr{H}}: \mathcal{D}_{\text {poly }}^{k-1} \rightarrow \mathcal{D}_{\text {poly }}^{k}$ defined by

$$
\begin{array}{r}
d_{\mathscr{H}}\left(u_{1} \otimes \cdots \otimes u_{k}\right)=1 \otimes u_{1} \otimes \cdots \otimes u_{k}+\sum_{i=1}^{k}(-1)^{i} u_{1} \otimes \cdots \otimes u_{i-1} \otimes \Delta\left(u_{i}\right) \otimes u_{i+1} \otimes \cdots \otimes u_{k} \\
+(-1)^{k+1} u_{1} \otimes \cdots \otimes u_{k} \otimes 1 .
\end{array}
$$

Since the comultiplication $\Delta: \mathcal{D}_{\text {poly }}^{0} \rightarrow \mathcal{D}_{\text {poly }}^{0} \otimes_{R} \mathcal{D}_{\text {poly }}^{0}$ is cocommutative and coassociative, $d_{\mathscr{H}}$ is a coboundary operator, i.e. $d_{\mathscr{H}}^{2}=0$. Moreover, since the comultiplication $\Delta$ is a morphism of $\mathcal{U}(A)$ modules, $d_{\mathscr{H}}: \mathcal{D}_{\text {poly }}^{k-1} \rightarrow \mathcal{D}_{\text {poly }}^{k}$ is a morphism of $\mathcal{U}(A)$-modules. Therefore, the Hochschild complex

$$
0 \longrightarrow \mathcal{D}_{\text {poly }}^{-1} \stackrel{d_{\mathscr{H}}}{\longrightarrow} \mathcal{D}_{\text {poly }}^{0} \stackrel{d_{\mathscr{H}}}{\longrightarrow} \mathcal{D}_{\text {poly }}^{1} \stackrel{d_{\mathscr{H}}}{\longrightarrow} \mathcal{D}_{\text {poly }}^{2} \stackrel{d_{\mathscr{H}}}{\longrightarrow} \cdots
$$

is a complex of $\mathcal{U}(A)$-modules.

The Chevalley-Eilenberg cohomology $\mathbb{H}_{\mathrm{CE}}^{k}\left(A, \mathcal{D}_{\text {poly }}^{\bullet}\right)$ in degree $k$ of the Hochschild complex of the pair $(L, A)$ is the degree $k$ total cohomology of the double complex

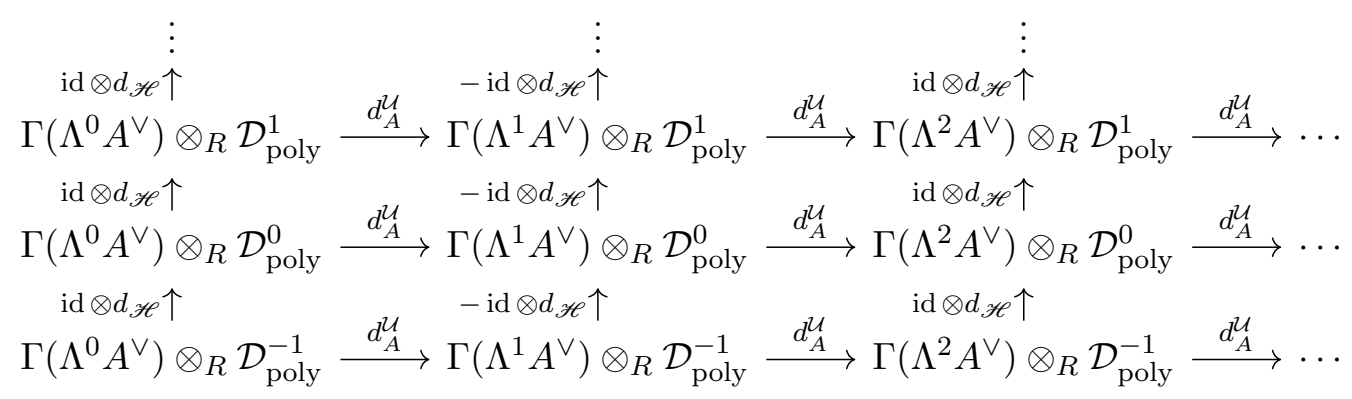


The coboundary operator $d_{A}^{\mathcal{U}}: \Gamma\left(\Lambda^{p} A^{\vee}\right) \otimes_{R} \mathcal{D}_{\text {poly }}^{q} \rightarrow \Gamma\left(\Lambda^{p+1} A^{\vee}\right) \otimes_{R} \mathcal{D}_{\text {poly }}^{q}$ is defined by

$$
\begin{aligned}
d_{A}^{\mathcal{U}}\left(\omega \otimes u_{0} \otimes \cdots \otimes u_{q}\right)= & \left(d_{A} \omega\right) \otimes u_{0} \otimes \cdots \otimes u_{q} \\
& +\sum_{j=1}^{\operatorname{rk}(A)} \sum_{k=0}^{q}\left(\alpha_{j} \wedge \omega\right) \otimes u_{0} \otimes \cdots \otimes u_{k-1} \otimes a_{j} \cdot u_{k} \otimes u_{k+1} \otimes \cdots \otimes u_{q},
\end{aligned}
$$

for all $\omega \in \Gamma\left(\Lambda^{p} A^{\vee}\right)$ and $u_{0}, u_{1}, \ldots, u_{q} \in \mathcal{D}_{\text {poly }}^{0}$ - for $q=-1$, we simply have $d_{A}^{\mathcal{U}}=d_{A}$. Here $\left(a_{i}\right)_{i \in\{1, \ldots, r\}}$ is any local frame of $A$ and $\left(\alpha_{j}\right)_{j \in\{1, \ldots, r\}}$ is the dual local frame of $A^{\vee}$. In other words, $\mathbb{H}_{\mathrm{CE}}^{k}\left(A, \mathcal{D}_{\text {poly }}^{\bullet}\right)$ is the cohomology of the total complex

$$
\left(\operatorname{tot}\left(\Gamma\left(\Lambda^{\bullet} A^{\vee}\right) \otimes_{R} \mathcal{D}_{\text {poly }}^{\bullet}\right), d_{A}^{\mathcal{U}}+\mathfrak{d}_{\mathscr{H}}\right),
$$

where we use the abbreviated symbol $\mathfrak{d}_{\mathscr{H}}$ to denote the operator id $\otimes d_{\mathscr{H}}$. See Equation (7) for the sign convention used in the definition of the map id $\otimes d_{\mathscr{H}}$.

However, unlike the universal enveloping algebra of a Lie algebroid, $\mathcal{D}_{\text {poly }}^{0}$ is in general not a Hopf algebroid over $R$ - in fact, $\mathcal{D}_{\text {poly }}^{0}$ is not even an associative algebra. Therefore, a priori, the Hochschild cohomology is only a vector space.

Remark 1.2. In general $\mathcal{D}_{\text {poly }}^{0}=\frac{\mathcal{U}(L)}{\mathcal{U}(L) \Gamma(A)}$ does not admit an associative product. For a Lie pair $\left(T_{M}, T_{F}\right)$ encoding a foliation $F$ on a smooth manifold $M$, Vitagliano proved that $\Gamma\left(\Lambda^{\bullet} A^{\vee}\right) \otimes_{R} \mathcal{D}_{\text {poly }}^{0}$ can be thought of as the space of normal differential operators of the foliation $F$ and admits an $A_{\infty^{-}}$ algebra structure [48]. For a generic Lie pair $(L, A)$, the existence of an $A_{\infty}$-algebra structure on $\Gamma\left(\Lambda^{\bullet} A^{\vee}\right) \otimes_{R} \mathcal{D}_{\text {poly }}^{0}$ was proved in [43].

There is a natural cup product

$$
\left(\Gamma\left(\Lambda^{k} A^{\vee}\right) \otimes_{R} \mathcal{D}_{\text {poly }}^{p}\right) \otimes\left(\Gamma\left(\Lambda^{l} A^{\vee}\right) \otimes_{R} \mathcal{D}_{\text {poly }}^{q}\right) \leftrightarrows \Gamma\left(\Lambda^{k+l} A^{\vee}\right) \otimes_{R} \mathcal{D}_{\text {poly }}^{p+q+1}
$$

on tot $\left(\Gamma\left(\Lambda^{\bullet} A^{\vee}\right) \otimes_{R} \mathcal{D}_{\text {poly }}^{\bullet}\right)$ defined by

$$
(\omega \otimes u) \smile(\theta \otimes v)=(-1)^{l(p+1)}(\omega \wedge \theta) \otimes(u \otimes v)
$$

for all $\omega \in \Gamma\left(\Lambda^{k} A^{\vee}\right), \theta \in \Gamma\left(\Lambda^{l} A^{\vee}\right), u \in \mathcal{D}_{\text {poly }}^{p}$ and $v \in \mathcal{D}_{\text {poly }}^{q}$.

The following proposition is easily verified.

Lemma 1.3. For any Lie pair $(L, A)$, the cochain complex $\left(\operatorname{tot}\left(\Gamma\left(\Lambda^{\bullet} A^{\vee}\right) \otimes_{R} \mathcal{D}_{\text {poly }}^{\bullet}\right), d_{A}^{\mathcal{U}}+\mathfrak{d} \mathscr{H}\right)$, equipped with the cup product (8), is a dg associative algebra. Therefore, there is an induced associative algebra structure on the Hochschild cohomology $\mathbb{H}_{\mathrm{CE}}^{\bullet}\left(A, \mathcal{D}_{\text {poly }}^{\bullet}\right)$.

Remark 1.4. It is natural to expect that the induced associative product on $\mathbb{H}_{\mathrm{CE}}^{\bullet}\left(A, \mathcal{D}_{\text {poly }}^{\bullet}\right)$ is graded commutative, as in the case of the usual Hochschild cohomology $H^{\bullet}\left(\mathcal{D}_{\text {poly }}^{\bullet}(M), d_{\mathscr{H}}\right)$ associated to a smooth manifold $M$. The group $H^{\bullet}\left(\mathcal{D}_{\text {poly }}^{\bullet}(M), d_{\mathscr{H}}\right)$ is the cohomology of a subcomplex of the Hochschild cochain complex $C^{\bullet}\left(C^{\infty}(M), C^{\infty}(M)\right)$. Its cup product is graded commutative up to a homotopy given by the Gerstenhaber pre-Lie bracket [19, Theorem 3]. This pre-Lie bracket can be defined in terms of the comultiplication and the multiplication on $\mathcal{D}_{\text {poly }}^{0}(M)$ : see Equation (21) for the formula in a similar situation. However, this approach does not work for a Lie pair $(L, A)$, since $\mathcal{D}_{\text {poly }}^{0}=\frac{\mathcal{U}(L)}{\mathcal{U}(L) \Gamma(A)}$ does not admit an associative multiplication, thus the usual proof for graded commutativity does not extend to our situation. In what follows, we will get around this difficulty by establishing an isomorphism of associative algebras between $\mathbb{H}_{\mathrm{CE}}^{\bullet}\left(A, \mathcal{D}_{\text {poly }}^{\bullet}\right)$ and the Hochschild cohomology of the Fedosov dg Lie algebroid of the Lie pair $(L, A)$ - see Proposition 3.18 and Proposition 3.24. The graded commutativity of the latter can be proved the usual way - see Proposition 2.8. 
1.3. Polyvector fields. Likewise, given a Lie pair $(L, A)$, let $\mathcal{T}_{\text {poly }}^{-1}$ denote the algebra $R$ of smooth functions on the manifold $M$ and set $\mathcal{T}_{\text {poly }}^{k}=\Gamma\left(\Lambda^{k+1}(L / A)\right)$ for $k \geqslant 0$. Consider $\mathcal{T}_{\text {poly }}^{\bullet}=\bigoplus_{k=-1}^{\infty} \mathcal{T}_{\text {poly }}^{k}$ as a complex of $\mathcal{U}(A)$-modules with trivial differential:

$$
0 \longrightarrow \mathcal{T}_{\text {poly }}^{-1} \stackrel{0}{\longrightarrow} \mathcal{T}_{\text {poly }}^{0} \stackrel{0}{\longrightarrow} \mathcal{T}_{\text {poly }}^{1} \stackrel{0}{\longrightarrow} \mathcal{T}_{\text {poly }}^{2} \stackrel{0}{\longrightarrow} \cdots
$$

Its Chevalley-Eilenberg cohomology $\mathbb{H}_{\mathrm{CE}}^{k}\left(A, \mathcal{T}_{\text {poly }}^{\bullet}\right)$ in degree $k$ is the degree $k$ total cohomology of the double complex

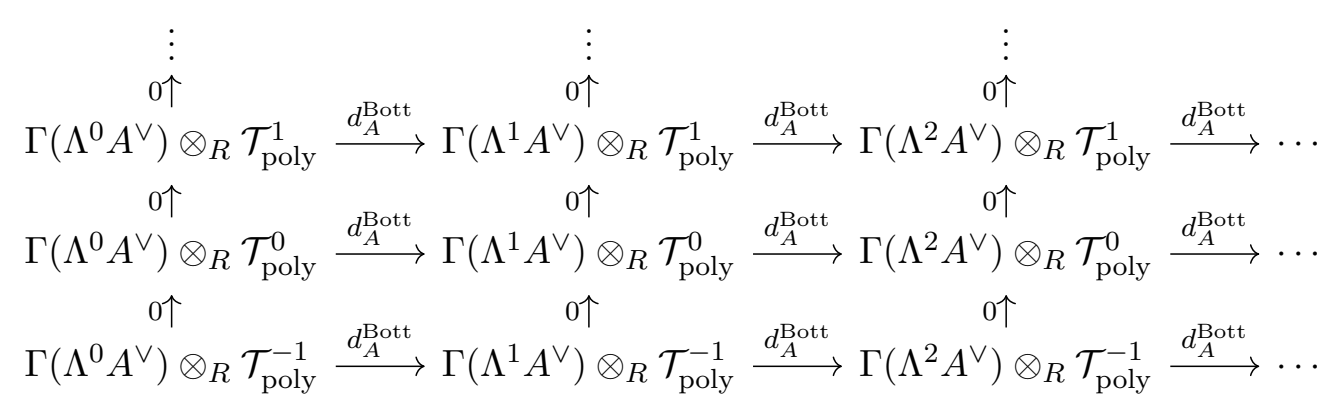

The coboundary operator $d_{A}^{\text {Bott }}: \Gamma\left(\Lambda^{p} A^{\vee}\right) \otimes \mathcal{T}_{\text {poly }}^{q} \rightarrow \Gamma\left(\Lambda^{p+1} A^{\vee}\right) \otimes \mathcal{T}_{\text {poly }}^{q}$ is defined by

$$
\begin{aligned}
d_{A}^{\text {Bott }}\left(\omega \otimes b_{0} \wedge \cdots \wedge b_{q}\right)= & \left(d_{A} \omega\right) \otimes b_{0} \wedge \cdots \wedge b_{q} \\
& +\sum_{j=1}^{\operatorname{rk}(A)} \sum_{k=0}^{q}\left(\alpha_{j} \wedge \omega\right) \otimes b_{0} \wedge \cdots \wedge b_{k-1} \wedge \nabla_{a_{j}}^{\mathrm{Bott}} b_{k} \wedge b_{k+1} \wedge \cdots \wedge b_{q},
\end{aligned}
$$

for all $\omega \in \Gamma\left(\Lambda^{p} A^{\vee}\right)$ and $b_{0}, b_{1}, \ldots, b_{q} \in \Gamma(L / A)$. Here $\left(a_{i}\right)_{i \in\{1, \ldots, r\}}$ is any local frame of $A$ and $\left(\alpha_{j}\right)_{j \in\{1, \ldots, r\}}$ is the dual local frame of $A^{\vee}$.

There is a natural wedge product

$$
\left(\Gamma\left(\Lambda^{k} A^{\vee}\right) \otimes_{R} \mathcal{T}_{\text {poly }}^{p}\right) \otimes\left(\Gamma\left(\Lambda^{l} A^{\vee}\right) \otimes_{R} \mathcal{T}_{\text {poly }}^{q}\right) \stackrel{\wedge}{\rightarrow} \Gamma\left(\Lambda^{k+l} A^{\vee}\right) \otimes_{R} \mathcal{T}_{\text {poly }}^{p+q+1}
$$

on tot $\left(\Gamma\left(\Lambda^{\bullet} A^{\vee}\right) \otimes_{R} \mathcal{T}_{\text {poly }}^{\bullet}\right)$ defined by

$$
(\omega \otimes u) \wedge(\theta \otimes v)=(-1)^{l(p+1)}(\omega \wedge \theta) \otimes(u \otimes v)
$$

for all $\omega \in \Gamma\left(\Lambda^{k} A^{\vee}\right), \theta \in \Gamma\left(\Lambda^{l} A^{\vee}\right), u \in \mathcal{T}_{\text {poly }}^{p}$ and $v \in \mathcal{T}_{\text {poly }}^{q}$.

We have the following

Lemma 1.5. For any Lie pair $(L, A)$, the cochain complex $\left(\operatorname{tot}\left(\Gamma\left(\Lambda^{\bullet} A^{\vee}\right) \otimes_{R} \mathcal{T}_{\text {poly }}^{\bullet}\right), d_{A}^{\text {Bott }}\right)$, equipped with the wedge product (10), is a dg commutative algebra. Therefore, the cohomology $\mathbb{H}_{\mathrm{CE}}^{\bullet}\left(A, \mathcal{T}_{\text {poly }}^{\bullet}\right)$ is a graded commutative algebra.

\section{Fedosov DG Lie Algebroids}

2.1. Dg Lie algebroids and polyvector fields and polydifferential operators. A $\mathbb{Z}$-graded manifold $\mathcal{M}$ with base manifold $M$ is a sheaf $\mathcal{A}$ of $\mathbb{Z}$-graded commutative $\mathcal{O}_{M}$-algebras over $M$ such that there exists a $\mathbb{Z}$-graded vector space $V$, a covering of $M$ by open submanifolds $U \subset M$, and a collection of isomorphisms of $C^{\infty}(U, \mathbb{k})$-algebras

$$
\left.\mathcal{A}\right|_{U} \cong C^{\infty}(U, \mathbb{k}) \otimes_{\mathbb{k}} \widehat{S}\left(V^{\vee}\right)
$$

where $\widehat{S}\left(V^{\vee}\right)$ denotes the $\mathbb{k}$-algebra of formal power series on $V$. Here $\mathcal{O}_{M}$ denotes the sheaf of $\mathbb{k}$-valued $C^{\infty}$ functions over $M$. By $C^{\infty}(\mathcal{M})$, we denote the $\mathbb{Z}$-graded commutative algebra $\Gamma(M, \mathcal{A})$ of global 
sections of $(M, \mathcal{A})$. By a dg manifold, we mean a $\mathbb{Z}$-graded manifold $\mathcal{M}$ endowed with a homological vector field, i.e. a derivation $Q$ of degree +1 of $C^{\infty}(\mathcal{M})$ satisfying $[Q, Q]=0$.

Example 2.1. Let $A \rightarrow M$ be a Lie algebroid over $\mathbb{k}$. Then $A[1]$ is a dg manifold with the ChevalleyEilenberg differential $d_{\mathrm{CE}}$ as homological vector field. According to Vainntrob [47], there is a bijection between the Lie algebroid structures on the vector bundle $A \rightarrow M$ and the homological vector fields on the $\mathbb{Z}$-graded manifold $A[1]$.

Example 2.2. Let $\mathfrak{g}=\bigoplus_{i \in \mathbb{Z}} \mathfrak{g}_{i}$ be a $\mathbb{Z}$-graded finite dimensional vector space. Then the graded manifold $\mathfrak{g}[1]$ is a dg manifold, if and only if the graded vector space $\mathfrak{g}$ admits a structure of curved $L_{\infty}$ algebra.

Below we recall some basic notations regarding dg vector bundles. For details, see [37, 36, 27, 44]. A dg vector bundle is a vector bundle object in the category of $\mathrm{dg}$ manifolds. Consider a vector bundle object $\mathcal{E} \stackrel{\pi}{\rightarrow} \mathcal{M}$ in the category of $\mathbb{Z}$-graded manifolds. Its space of sections $\Gamma(\mathcal{E})$ is defined to be the direct sum $\bigoplus_{j \in \mathbb{Z}} \Gamma(\mathcal{E})^{j}$, where $\Gamma(\mathcal{E})^{j}$ consists of the sections of degree $j$, i.e. the maps $l \in \operatorname{Hom}(\mathcal{M}, \mathcal{E}[-j])$ such that $(\pi[-j]) \circ l=\operatorname{id}_{\mathcal{M}}$. Here $\pi[-j]: \mathcal{E}[-j] \rightarrow \mathcal{M}$ is the natural map induced from $\pi$ - see [37, 36] for more details.

Remark 2.3. When $\mathcal{E} \rightarrow \mathcal{M}$ is a dg vector bundle, the homological vector fields on $\mathcal{E}$ and $\mathcal{M}$ naturally induce a degree $(+1)$ operator $\mathcal{Q}$ on $\Gamma(\mathcal{E})$, making $\Gamma(\mathcal{E})$ a dg module over $C^{\infty}(\mathcal{M})$. Since the space $\Gamma\left(\mathcal{E}^{\vee}\right)$ of linear functions on $\mathcal{E}$ and the pull-back of $C^{\infty}(\mathcal{M})$ via $\pi$ together generate $C^{\infty}(\mathcal{E})$, the converse is also true - see [38].

Example 2.4. Let $(\mathcal{M}, Q)$ be a dg manifold. The space $\mathfrak{X}(\mathcal{M})$ of vector fields on $\mathcal{M}$ (i.e. graded derivations of $C^{\infty}(\mathcal{M})$ ), which can be regarded as the space of sections $\Gamma\left(T_{\mathcal{M}}\right)$, is naturally a $d g$ module over the dg algebra $\left(C^{\infty}(\mathcal{M}), Q\right)$ with the Lie derivative $\mathcal{L}_{Q}: \mathfrak{X}(\mathcal{M}) \rightarrow \mathfrak{X}(\mathcal{M})$ playing the role of the operator $\mathcal{Q}$. As a consequence, $T_{\mathcal{M}}$ is a dg manifold - the homological vector field on $T_{\mathcal{M}}$ is called the complete lift of $Q$ as well as tangent lift in [38] — and $T_{\mathcal{M}} \rightarrow \mathcal{M}$ is a dg vector bundle.

The following lemma is standard [38].

Lemma 2.5. Assume $\mathcal{E}$ is a dg vector bundle over the $d g$ manifold $(\mathcal{M}, Q)$.

(1) Then the dual bundle $\mathcal{E}^{\vee}$ is a dg vector bundle over $(\mathcal{M}, Q)$.

(2) Furthermore, for all $k \geqslant 1$, the exterior tensor power vector bundle $\Lambda^{k} \mathcal{E}$ is a dg vector bundle over $(\mathcal{M}, Q)$.

Here and throughout the paper, we use the shorthand notation $\Lambda^{k} \mathcal{E}$ abusively to actually denote $\left(S^{k}(\mathcal{E}[-1])\right)[k]$.

Proof. By assumption, $\Gamma(\mathcal{E})$ is a dg module over $\left(C^{\infty}(\mathcal{M}), Q\right)$ with degree $(+1)$ differential $\mathcal{Q}: \Gamma(\mathcal{E}) \rightarrow \Gamma(\mathcal{E})$. Define a degree $(+1)$ operator $\mathcal{Q}: \Gamma\left(\mathcal{E}^{\vee}\right) \rightarrow \Gamma\left(\mathcal{E}^{\vee}\right)$ by

$$
\langle\mathcal{Q}(\xi) \mid l\rangle=Q\langle\xi \mid l\rangle-(-1)^{|\xi|}\langle\xi \mid \mathcal{Q}(l)\rangle
$$

for all homogeneous $\xi \in \Gamma\left(\mathcal{E}^{\vee}\right)$ and $l \in \Gamma(\mathcal{E})$. It is simple to see that this operator makes $\Gamma\left(\mathcal{E}^{\vee}\right)$ into a dg module over $\left(C^{\infty}(\mathcal{M}), Q\right)$.

Similarly, $\Gamma\left(\Lambda^{k} \mathcal{E}\right)$ is a dg module over $\left(C^{\infty}(\mathcal{M}), Q\right)$ with the differential $\mathcal{Q}: \Gamma\left(\Lambda^{k} \mathcal{E}\right) \rightarrow \Gamma\left(\Lambda^{k} \mathcal{E}\right)$ of degree $(+1)$ defined by

$$
\mathcal{Q}\left(l_{1} \wedge \cdots \wedge l_{k}\right)=\sum_{i=1}^{k}(-1)^{\left|l_{1}\right|+\cdots\left|l_{i-1}\right|} l_{1} \wedge \cdots \wedge \mathcal{Q}\left(l_{i}\right) \wedge \cdots \wedge l_{k}
$$

for all homogeneous $l_{1}, \ldots, l_{k} \in \Gamma(\mathcal{E})$.

The conclusion thus follows. 
A dg Lie algebroid is a Lie algebroid object in the category of dg manifolds. Equivalently, a dg Lie algebroid is a dg vector bundle $\mathcal{L} \rightarrow \mathcal{M}$ endowed with a $\mathbb{Z}$-graded Lie algebroid structure satisfying the compatibility condition

$$
\left[d_{\mathcal{L}}, \mathcal{Q}\right]=0
$$

where $d_{\mathcal{L}}$ is the Chevalley-Eilenberg differential

$$
d_{\mathcal{L}}: \Gamma\left(\Lambda^{\bullet} \mathcal{L}^{\vee}\right) \rightarrow \Gamma\left(\Lambda^{\bullet+1} \mathcal{L}^{\vee}\right)
$$

of the Lie algebroid $\mathcal{L} \rightarrow \mathcal{M}, \mathcal{Q}$ is the differential (of internal degree $(+1)$ )

$$
\mathcal{Q}: \Gamma\left(\Lambda^{\bullet} \mathcal{L}^{\vee}\right) \rightarrow \Gamma\left(\Lambda^{\bullet} \mathcal{L}^{\vee}\right)
$$

induced by the $\mathrm{dg}$ vector bundle structure on $\mathcal{L} \rightarrow \mathcal{M}$ (see Lemma 2.5), and [-,-] denotes the commutator. For more details, we refer the reader to $[36,37]$, where dg Lie algebroids are called $Q$-algebroids.

Example 2.6. As in Example 2.4, let $(\mathcal{M}, Q)$ be a dg manifold. In addition to being a dg vector bundle, $T_{\mathcal{M}} \rightarrow \mathcal{M}$ is also a Lie algebroid. In this case, the Chevalley-Eilenberg differential (13) is the de Rham differential

$$
d_{\mathrm{DR}}: \Omega^{\bullet}(\mathcal{M}) \rightarrow \Omega^{\bullet+1}(\mathcal{M}),
$$

while the internal differential (14) is the Lie derivative

$$
\mathcal{L}_{Q}: \Omega^{\bullet}(\mathcal{M}) \rightarrow \Omega^{\bullet}(\mathcal{M}) .
$$

Since $\left[d_{\mathrm{DR}}, \mathcal{L}_{Q}\right]=0$, it follows that $T_{\mathcal{M}}$ is indeed a $d g$ Lie algebroid.

For an ordinary $\mathbb{Z}$-graded Lie algebroid, one can speak about "polyvector fields" and "polydifferential operators" on the Lie algebroid. For a $\mathrm{dg}$ Lie algebroid, the dg structure will induce degree $(+1)$ differentials on "polyvector fields" and "polydifferential operators". For instance, the "polyvector fields" and "polydifferential operators" for the tangent $\mathrm{dg}$ Lie algebroid $T_{\mathcal{M}}$ of a $\operatorname{dg}$ manifold $(\mathcal{M}, Q)$ as in Example 2.6 are, respectively, the polyvector fields and the polydifferential operators on $\mathcal{M}$, while the induced degree $(+1)$ differentials are $\mathcal{L}_{Q}$ and $\llbracket Q,-\rrbracket$, respectively. Here $\llbracket-,-\rrbracket$ stands for the Gerstenhaber bracket on the polydifferential operators of $\mathcal{M}$.

More precisely, a $k$-vector field on a dg Lie algebroid $\mathcal{L} \rightarrow \mathcal{M}$ is a section of the vector bundle $\Lambda^{k} \mathcal{L} \rightarrow \mathcal{M}$. Since $\mathcal{L} \rightarrow \mathcal{M}$ is a dg vector bundle, according to Lemma 2.5, we have a degree $(+1)$ differential $\mathcal{Q}: \Gamma\left(\Lambda^{k+1} \mathcal{L}\right) \rightarrow \Gamma\left(\Lambda^{k+1} \mathcal{L}\right)$ - see Equation (11). On the other hand, the Lie algebroid structure on $\mathcal{L}$ yields a Schouten bracket

$$
[-,-]: \Gamma\left(\Lambda^{u+1} \mathcal{L}\right) \otimes \Gamma\left(\Lambda^{v+1} \mathcal{L}\right) \rightarrow \Gamma\left(\Lambda^{u+v+1} \mathcal{L}\right) .
$$

For $n \in \mathbb{Z}$, we set

$$
\operatorname{tot}_{\oplus}^{n} \Gamma\left(\Lambda^{\bullet+1} \mathcal{L}\right)=\bigoplus_{\substack{p+q=n \\ p, q \in \mathbb{Z} \\ q \geqslant-1}}\left(\Gamma\left(\Lambda^{q+1} \mathcal{L}\right)\right)^{p},
$$

where $\left(\Gamma\left(\Lambda^{q+1} \mathcal{L}\right)\right)^{p}$ denotes the subspace of $\Gamma\left(\Lambda^{q+1} \mathcal{L}\right)$ consisting of homogeneous elements of degree $p+q$.

Proposition 2.7. Let $\mathcal{L}$ be a dg Lie algebroid over $\mathcal{M}$.

(1) When endowed with the differential $\mathcal{Q}$, the wedge product, and the Schouten bracket, the space of 'polyvector fields' tot $\oplus \Gamma\left(\Lambda^{\bullet+1} \mathcal{L}\right)$ is a differential Gerstenhaber algebra' 1 whence a dgla.

(2) When endowed with the wedge product and the Schouten bracket, the cohomology $\mathbb{H}^{\bullet}\left(\operatorname{tot}_{\oplus} \Gamma\left(\Lambda^{\bullet+1} \mathcal{L}\right), \mathcal{Q}\right)$ is a Gerstenhaber algebra.

\footnotetext{
${ }^{1}$ Here and in the sequel, by a differential Gerstenhaber algebra, we mean a Gerstenhaber algebra equipped with a degree $(+1)$ differential, which is a derivation of both the associative multiplication and the Lie bracket. Such structures were called strongly differential Gerstenhaber algebras in $[54,26]$.
} 
Similarly, a $k$-differential operator for a Lie algebroid $\mathcal{L}$ is an element of $s \mathcal{U}(\mathcal{L})^{\otimes k}$, the tensor product (as left $C^{\infty}(\mathcal{M})$-modules) of $k$ copies of the suspended universal enveloping algebra $s \mathcal{U}(\mathcal{L})$. Recall that the universal enveloping algebra $\mathcal{U}(\mathcal{L})$ of a $\mathbb{Z}$-graded Lie algebroid $\mathcal{L} \rightarrow \mathcal{M}$ with anchor $\varrho: \mathcal{L} \rightarrow T_{\mathcal{M}}$ is the quotient of the (reduced) tensor algebra

$$
\bigoplus_{n=1}^{\infty}(\mathcal{R} \oplus \Gamma(\mathcal{L}))^{\otimes n}
$$

of the $\mathbb{k}$-module $\mathcal{R} \oplus \Gamma(\mathcal{L})$ by the two-sided ideal generated by the elements of the following four types:

$$
\begin{array}{ll}
X \otimes Y-(-1)^{|X||Y|} Y \otimes X-[X, Y] & f \otimes X-f X \\
X \otimes g-(-1)^{|g||X|} g \otimes X-\varrho_{X}(g) & f \otimes g-f g
\end{array}
$$

for all homogeneous $X, Y \in \Gamma(\mathcal{L})$ and $f, g \in \mathcal{R}$. As earlier, the symbol $\mathcal{R}$ denotes $C^{\infty}(\mathcal{M})$.

The universal enveloping algebra $\mathcal{U}(\mathcal{L})$ is a coalgebra over $\mathcal{R}$ [55]. Its comultiplication

$$
\Delta: \mathcal{U}(\mathcal{L}) \rightarrow \mathcal{U}(\mathcal{L}) \otimes_{\mathcal{R}} \mathcal{U}(\mathcal{L})
$$

is an $R$-linear map of degree 0 characterized by the identities

$$
\begin{gathered}
\Delta(1)=1 \otimes 1 \\
\Delta(b)=1 \otimes b+b \otimes 1, \quad \forall b \in \Gamma(\mathcal{L}) ; \\
\Delta(u \cdot v)=\Delta(u) \cdot \Delta(v), \quad \forall u, v \in \mathcal{U}(\mathcal{L}),
\end{gathered}
$$

where the symbol $\cdot$ denotes the multiplication in $\mathcal{U}(\mathcal{L})$. We refer the reader to [55] for the precise meaning of (the r.h.s. of) the last equation above. More explicitly, we have

$$
\begin{aligned}
\Delta\left(b_{1} b_{2} \cdots b_{n}\right)=1 \otimes\left(b_{1} b_{2} \cdots b_{n}\right)+\sum_{p=1}^{n-1} \sum_{\sigma \in \mathfrak{S}_{p}^{n-p}} \pm\left(b_{\sigma(1)} \cdots b_{\sigma(p)}\right) \otimes\left(b_{\sigma(p+1)} \cdots\right. & \left.b_{\sigma(n)}\right) \\
& +\left(b_{1} b_{2} \cdots b_{n}\right) \otimes 1,
\end{aligned}
$$

where \pm denotes the Koszul sign of the $(p, n-p)$-shuffle ${ }^{2} \sigma$ of the $n$-tuple of homogeneous elements $b_{1}, \ldots, b_{n}$ of $\Gamma(\mathcal{L})$.

Now assume that $\mathcal{L} \rightarrow \mathcal{M}$ is a dg Lie algebroid. The differential $\mathcal{Q}: \Gamma(\mathcal{L}) \rightarrow \Gamma(\mathcal{L})$ and the homological vector field $Q: C^{\infty}(\mathcal{M}) \rightarrow C^{\infty}(\mathcal{M})$ induce a differential of degree $(+1)$ on the (reduced) tensor algebra (15) by way of the Leibniz rule. From the compatibility condition (12), it is simple to see that the two-sided ideal generated by the elements (16) is stable under this induced differential on the universal enveloping algebra

$$
\mathcal{Q}: \mathcal{U}(\mathcal{L}) \rightarrow \mathcal{U}(\mathcal{L})
$$

which we denote by the same symbol $\mathcal{Q}$ by abuse of notation. This differential is compatible with both the algebra and coalgebra structures on $\mathcal{U}(\mathcal{L})$ so that $\mathcal{U}(\mathcal{L})$ is a dg Hopf algebroid over the dga $\mathcal{R}=C^{\infty}(\mathcal{M})$. As a consequence, we obtain a differential $\mathcal{Q}: s \mathcal{U}(\mathcal{L})^{\otimes k+1} \rightarrow s \mathcal{U}(\mathcal{L})^{\otimes k+1}$ of degree $(+1)$ for each $k \geqslant-1$. Here $s \mathcal{U}(\mathcal{L})^{\otimes 0}=s \mathcal{R}$ and $s \mathcal{U}(\mathcal{L})^{\otimes k+1}$ (with $k \geqslant 0$ ) denotes the tensor product $s \mathcal{U}(\mathcal{L}) \otimes_{\mathcal{R}} \cdots \otimes_{\mathcal{R}} s \mathcal{U}(\mathcal{L})$ of $(k+1)$-copies of the left $\mathcal{R}$-module $s \mathcal{U}(\mathcal{L})$.

A Hochschild coboundary differential

$$
d_{\mathscr{H}}: s \mathcal{U}(\mathcal{L})^{\otimes k} \rightarrow s \mathcal{U}(\mathcal{L})^{\otimes k+1}
$$

and Gerstenhaber bracket

$$
\llbracket-,-\rrbracket: s \mathcal{U}(\mathcal{L})^{\otimes p+1} \otimes s \mathcal{U}(\mathcal{L})^{\otimes q+1} \rightarrow s \mathcal{U}(\mathcal{L})^{\otimes p+q+1}
$$

${ }^{2} \mathrm{~A}(p, q)$-shuffle is a permutation $\sigma \in S_{p+q}$ of the set $\{1,2, \cdots, p+q\}$ satisfying $\sigma(1)<\sigma(2)<\cdots<\sigma(p)$ and $\sigma(p+1)<\sigma(p+2)<\cdots<\sigma(p+q)$. The subset of $S_{p+q}$ consisting of all $(p, q)$-shuffles is denoted $\mathfrak{S}_{p}^{q}$. 
can be defined by the following explicit algebraic identities:

$$
\begin{aligned}
& d_{\mathscr{H}}\left(u_{1} \otimes \cdots \otimes u_{k}\right)=( \pm) 1 \otimes u_{1} \otimes \cdots \otimes u_{k}+\sum_{i=1}^{k}( \pm) u_{1} \otimes \cdots \otimes \Delta\left(u_{i}\right) \otimes \cdots \otimes u_{k} \\
&+( \pm) u_{1} \otimes \cdots \otimes u_{k} \otimes 1
\end{aligned}
$$

and

$$
\llbracket \phi, \psi \rrbracket=\phi \star \psi-( \pm) \psi \star \phi,
$$

where $\phi \star \psi \in s \mathcal{U}(\mathcal{L})^{\otimes p+q+1}$ is defined by

$$
\phi \star \psi=\sum_{k=0}^{p}( \pm) u_{0} \otimes \cdots \otimes u_{k-1} \otimes\left(\hat{\Delta}^{q} u_{k}\right) \cdot \psi \otimes u_{k+1} \otimes \cdots \otimes u_{p}
$$

if $\phi=u_{0} \odot u_{1} \odot \cdots \odot u_{p}$ for some $u_{0}, u_{1}, \ldots, u_{p} \in s \mathcal{U}(\mathcal{L})$ and $\psi \in s \mathcal{U}(\mathcal{L})^{\otimes q+1}$. We refer the reader to [55] for the precise meaning of the product $\left(\hat{\Delta}^{q} u_{k}\right) \cdot \psi$ in $s \mathcal{U}(\mathcal{L})^{\otimes q+1}$ appearing in the last equation above. Here $\hat{\Delta}: s \mathcal{U}(\mathcal{L}) \rightarrow s \mathcal{U}(\mathcal{L}) \otimes_{\mathcal{R}} s \mathcal{U}(\mathcal{L})$ is the map induced by the comultiplication $\Delta$ on $\mathcal{U}(\mathcal{L})$.

Finally, the tensor algebra of $s \mathcal{U}(\mathcal{L})$ over $\mathcal{R}$ carries an obvious cup product

$$
s \mathcal{U}(\mathcal{L})^{\otimes p} \otimes s \mathcal{U}(\mathcal{L})^{\otimes q} \leftrightharpoons s \mathcal{U}(\mathcal{L})^{\otimes p+q}
$$

the tensor product over $\mathcal{R}$ itself:

$$
\phi \smile \psi=\phi \otimes \psi
$$

For $n \in \mathbb{Z}$, we set

$$
\operatorname{tot}_{\oplus}^{n} s \mathcal{U}(\mathcal{L})^{\otimes \bullet+1}=\bigoplus_{\substack{p+q=n \\ p, q \in \mathbb{Z} \\ q \geqslant-1}}\left(s \mathcal{U}(\mathcal{L})^{\otimes q+1}\right)^{p}
$$

where $\left(s \mathcal{U}(\mathcal{L})^{\otimes q+1}\right)^{p}$ denotes the subspace of $s \mathcal{U}(\mathcal{L})^{\otimes q+1}$ consisting of elements of degree $p+q$.

Proposition 2.8. Let $\mathcal{L}$ be a dg Lie algebroid over $\mathcal{M}$.

(1) When endowed with the differential $\mathcal{Q}+d_{\mathscr{H}}$ and the Gerstenhaber bracket $(19)$, $\operatorname{tot}_{\oplus} s \mathcal{U}(\mathcal{L})^{\otimes \bullet+1}$ is a dgla.

(2) When endowed with the cup product (i.e. the tensor product $\otimes_{\mathcal{R}}$ ) and the Gerstenhaber bracket, the Hochschild cohomology $\mathbb{H}^{\bullet}\left(\operatorname{tot}_{\oplus} s \mathcal{U}(\mathcal{L})^{\otimes \bullet+1}, \mathcal{Q}+d_{\mathscr{H}}\right)$ is a Gerstenhaber algebra.

Proof. This can be proved directly by adapting the ordinary Hochschild cohomology theory of associative algebras [19]. Since $\mathcal{U}(\mathcal{L})$ is a dg Hopf algebroid over $\mathcal{R}$, all relevant formulae in [19] concerning the algebraic structures on the Hochschild cochain complex of an associative algebras hold in our context, with the differential being $\mathcal{Q}+d_{\mathscr{H}}$, and the pre-Lie bracket and the cup product being given, respectively, by Equation (21) and Equation (22). We leave the details to the reader.

Remark 2.9. Contrary to Proposition 2.7, here $\operatorname{tot}_{\oplus} s \mathcal{U}(\mathcal{L})^{\otimes \bullet+1}$ is not a differential Gerstenhaber algebra, for the Lie bracket and the associative multiplication are only compatible up to homotopy. Likewise, the associative multiplication is graded commutative on the cohomology level, for the cup product on cochains is graded commutative only up to homotopy. This is reminiscent of the ordinary Hochschild cohomology theory of associative algebras — see [19]. 
2.2. Fedosov dg manifolds. In this section, we recall the basic construction of Fedosov dg manifolds of a Lie pair. For details, see [45].

Let $(L, A)$ be a Lie pair. We use the symbols $B$ to denote the quotient vector bundle $L / A$ and $r$ to denote its rank.

Consider the endomorphism $\delta$ of the vector bundle $\Lambda^{\bullet} L^{\vee} \otimes \hat{S} B^{\vee}$ defined by

$$
\delta\left(\omega \otimes \chi^{J}\right)=\sum_{m=1}^{r}\left(q^{\top}\left(\chi_{m}\right) \wedge \omega\right) \otimes J_{m} \chi^{J-e_{m}},
$$

for all $\omega \in \Lambda L^{\vee}$ and $J \in \mathbb{N}^{r}$. Here $\left\{\chi_{k}\right\}_{k=1}^{r}$ denotes an arbitrary local frame for the vector bundle $B^{\vee}$, the symbol $q^{\top}$ denotes the vector bundle morphism $q^{\top}: B^{\vee} \rightarrow L^{\vee}$ dual to the quotient morphism $q: L \rightarrow B$, the symbol $e_{m}$ denotes the multi-index $(0, \cdots, 0,1,0, \cdots, 0)$ having its single nonzero entry in the $m$-th position, and

$$
\chi^{J}=\underbrace{\chi_{1} \odot \cdots \odot \chi_{1}}_{J_{1} \text { factors }} \odot \underbrace{\chi_{2} \odot \cdots \odot \chi_{2}}_{J_{2} \text { factors }} \odot \cdots \odot \underbrace{\chi_{r} \odot \cdots \odot \chi_{r}}_{J_{r} \text { factors }}
$$

if $J=\left(J_{1}, J_{2}, \cdots, J_{r}\right)$.

The operator $\delta$ is a derivation of degree +1 of the bundle of graded commutative algebras $\Lambda^{\bullet} L^{\vee} \otimes \hat{S} B^{\vee}$ and satisfies $\delta^{2}=0$. The resulting cochain complex

$$
\cdots \rightarrow \Lambda^{n-1} L^{\vee} \otimes \hat{S} B^{\vee} \stackrel{\delta}{\longrightarrow} \Lambda^{n} L^{\vee} \otimes \hat{S} B^{\vee} \stackrel{\delta}{\longrightarrow} \Lambda^{n+1} L^{\vee} \otimes \hat{S} B^{\vee} \rightarrow \cdots
$$

admits a contraction onto the complex with trivial differential

$$
\cdots \longrightarrow \Lambda^{n-1} A^{\vee} \stackrel{0}{\longrightarrow} \Lambda^{n} A^{\vee} \stackrel{0}{\longrightarrow} \Lambda^{n+1} A^{\vee} \longrightarrow \cdots
$$

Indeed, for every choice of splitting $i \circ p+j \circ q=\mathrm{id}_{L}$ of the short exact sequence

$$
0 \longrightarrow A \underset{r_{-}--}{\stackrel{i}{\longrightarrow}} L \frac{q}{r_{j} \ldots-} B \longrightarrow 0
$$

and its dual

$$
0 \longrightarrow B^{\vee} \underset{j^{\top}}{\stackrel{q^{\top}}{\longrightarrow}} L^{\vee} \underset{p^{\top}}{\stackrel{i^{\top}}{\longrightarrow \ldots}} A^{\vee} \longrightarrow 0
$$

the chain maps

$$
\sigma: \Lambda^{\bullet} L^{\vee} \otimes \hat{S} B^{\vee} \rightarrow \Lambda^{\bullet} A^{\vee}
$$

and

$$
\tau: \Lambda^{\bullet} A^{\vee} \rightarrow \Lambda^{\bullet} L^{\vee} \otimes \hat{S} B^{\vee}
$$

respectively defined by

$$
\sigma\left(\omega \otimes \chi^{J}\right)= \begin{cases}i^{\top}(\omega) & \text { if }|J|=0 \\ 0 & \text { otherwise }\end{cases}
$$

for all $\omega \in \Lambda^{\bullet}\left(L^{\vee}\right)$, and

for all $\alpha \in \Lambda^{\bullet}\left(A^{\vee}\right)$, satisfy

$$
\tau(\alpha)=p^{\top}(\alpha) \otimes 1,
$$

where the homotopy operator

$$
\sigma \tau=\mathrm{id} \quad \text { and } \quad \mathrm{id}-\tau \sigma=h \delta+\delta h,
$$

$$
h: \Lambda^{\bullet} L^{\vee} \otimes \hat{S} B^{\vee} \rightarrow \Lambda^{\bullet-1} L^{\vee} \otimes \hat{S} B^{\vee}
$$

is defined by

$$
h\left(\omega \otimes \chi^{J}\right)= \begin{cases}\frac{1}{v+|J|} \sum_{k=1}^{r}\left(\iota_{j}\left(\partial_{k}\right) \omega\right) \otimes \chi^{J+e_{k}} & \text { if } v \geqslant 1 \\ 0 & \text { if } v=0\end{cases}
$$


for all $\omega \in p^{\top}\left(\Lambda^{u} A^{\vee}\right) \otimes q^{\top}\left(\Lambda^{v} B^{\vee}\right)$. Here $\left\{\partial_{k}\right\}_{k=1}^{r}$ denotes the local frame for $B$ dual to $\left\{\chi_{k}\right\}_{k=1}^{r}$. Notice that $h \tau=0, \sigma h=0$, and $h^{2}=0$, i.e. the triple of maps $(\tau, \sigma, h)$ make up a contraction of $\Lambda^{\bullet} L^{\vee} \otimes \hat{S} B^{\vee}$ onto $\Lambda^{\bullet} A^{\vee}$. We remark that the operator $h$ is not a derivation of the algebra $\Gamma\left(\Lambda^{\bullet} L^{\vee} \otimes \hat{S} B^{\vee}\right)$. However, the contraction $(\tau, \sigma, h)$ is compatible with the graded commutative algebra structures on $\Gamma\left(\Lambda^{\bullet} L^{\vee} \otimes \hat{S} B^{\vee}\right)$ and $\Gamma\left(\Lambda^{\bullet} A^{\vee}\right)$ in the following sense:

Lemma 2.10. The triple $(\tau, \sigma, h)$ is a semifull algebra contraction - see Definition A.2 - of $\Gamma\left(\Lambda^{\bullet} L^{\vee} \otimes \hat{S} B^{\vee}\right)$ onto $\Gamma\left(\Lambda^{\bullet} A^{\vee}\right)$. Furthermore, $\sigma$ and $\tau$ are morphisms of graded algebras.

Proof. The fact that $\tau$ and $\sigma$ are algebra morphisms follows directly from the definitions. Moreover, the last four identities in Definition A.2 follow at once from the fact that $\sigma$ is an algebra morphism (and the identities $\sigma h=0$ and $\sigma \tau=\mathrm{id}$ ). Denoting by $\mu$ the product on $\Gamma\left(\Lambda^{\bullet} L^{\vee} \otimes \hat{S} B^{\vee}\right)$, the remaining identities to prove are (recall that $\mu$ is graded commutative)

$$
h \mu(h \otimes h)=0, \quad h \mu(h \otimes \tau)=0, \quad h \mu(\tau \otimes \tau)=0 .
$$

To prove these, we introduce a second operator of degree $(-1)$

$$
\eta: \Lambda^{\bullet} L^{\vee} \otimes \hat{S} B^{\vee} \rightarrow \Lambda^{\bullet-1} L^{\vee} \otimes \hat{S} B^{\vee}
$$

defined by

$$
\eta\left(\omega \otimes \chi^{J}\right)=\sum_{k=1}^{r}\left(\iota_{j}\left(\partial_{k}\right) \omega\right) \otimes \chi^{J+e_{k}}
$$

for all $\omega \in \Lambda^{\bullet}\left(L^{\vee}\right)$. Notice the similarity with the definition of $h$. It is easy to check that $\eta$ is an algebra derivation and $\operatorname{ker}(\eta)=\operatorname{ker}(h)$. Using these two facts, we see that

$$
h^{2}=0 \quad \Rightarrow \quad \eta h=0 \quad \Rightarrow \quad \eta \mu(h \otimes h)=\mu(\eta \otimes \mathrm{id}+\mathrm{id} \otimes \eta)(h \otimes h)=0 \quad \Rightarrow \quad h \mu(h \otimes h)=0 .
$$

The remaining identities are proved in a similar way.

The notions of $L$-connection on $B$ extending the Bott $A$-connection and of torsion-free $L$-connection on $B$ were defined in $[11,29]$. A torsion-free $L$-connection on $B$ is necessarily an extension of the Bott $A$-connection [29, Lemma 5.2]. According to [45, Lemma 4.5], an $L$-connection $\nabla$ on $B$ is torsion-free if and only if

$$
\delta d_{L}^{\nabla}+d_{L}^{\nabla} \delta=0
$$

Theorem 2.11 ([45, Proposition 4.6]). Let $(L, A)$ be a Lie pair with quotient $B=L / A$. We interpret the sections of the bundle $L^{\vee} \otimes \hat{S} B^{\vee} \otimes B$ as derivations of the algebra $\Gamma\left(\Lambda^{\bullet} L^{\vee} \otimes \hat{S} B^{\vee}\right)$ in the natural way. Given a splitting of the short exact sequence (23) and a torsion-free $L$-connection $\nabla$ on $B$, there exists a unique derivation

$$
X^{\nabla} \in \Gamma\left(L^{\vee} \otimes \hat{S}^{\geqslant 2} B^{\vee} \otimes B\right)
$$

satisfying $\left(h \otimes \operatorname{id}_{B}\right)\left(X^{\nabla}\right)=0$ and such that the derivation

$$
Q: \Gamma\left(\Lambda^{\bullet} L^{\vee} \otimes \hat{S} B^{\vee}\right) \rightarrow \Gamma\left(\Lambda^{\bullet+1} L^{\vee} \otimes \hat{S} B^{\vee}\right)
$$

defined by

satisfies $Q^{2}=0$.

$$
Q=-\delta+d_{L}^{\nabla}+X^{\nabla}
$$

As a consequence, $\left(\mathcal{M}=L[1] \oplus B, Q=-\delta+d_{L}^{\nabla}+X^{\nabla}\right)$ is a dg manifold, which we call a Fedosov $d g$ manifold associated with the Lie pair $(L, A)$. The Fedosov $\operatorname{dg}$ manifold $(\mathcal{M}, Q)$ of Theorem 2.11 was also obtained independently by Batakidis-Voglaire [3] in the case of matched pairs.

Remark 2.12. The Kapranov dg manifold $A[1] \oplus L / A$ introduced in [29] is a dg submanifold of the Fedosov dg manifold $(L[1] \oplus L / A, Q)$. 
In order to study the dependence of the above construction on the involved choices, it is useful to review a different description of the Fedosov dg manifold, which can also be found in [45]. As shown in [28, 29] (see also [45, §3.4]), the choice of a splitting $j: B \rightarrow L$ of the short exact sequence $0 \rightarrow A \rightarrow L \rightarrow B \rightarrow 0$ and of an $L$-connection $\nabla$ on $B$ determines a Poincaré-Birkhoff-Witt isomorphism of filtered $C^{\infty}(M)$ coalgebras (PBW map in short)

$$
\text { pbw }: \Gamma(S B) \rightarrow \frac{\mathcal{U}(L)}{\mathcal{U}(L) \Gamma(A)}
$$

Since $\mathcal{U}(L) \Gamma(A)$ is a left ideal of the algebra $\mathcal{U}(L)$, there is a natural left $\Gamma(L)$-action on the quotient $\frac{\mathcal{U}(L)}{\mathcal{U}(L) \Gamma(A)}$, and an induced flat $L$-connection $\nabla^{\xi}$ on $S B$ :

$$
\nabla_{l}^{\natural}(s)=\mathrm{pbw}^{-1}(l \cdot \mathrm{pbw}(s))
$$

for all $l \in \Gamma(L)$ and $s \in \Gamma(S B)$. Moreover, for every $l \in \Gamma(L)$, the covariant derivative $\nabla_{l}^{\xi}$ is a coderivation of the $C^{\infty}(M)$-coalgebra $\Gamma(S B)$.

Dualizing, we obtain an $L$-connection on $\hat{S}\left(B^{\vee}\right)$, which we continue to denote by $\nabla^{\natural}$. Furthermore, for every $l \in \Gamma(L)$ the covariant derivative $\nabla_{l}^{\xi}$ is a derivation of the $C^{\infty}(M)$-algebra $\Gamma\left(\hat{S}\left(B^{\vee}\right)\right)$. Finally, this latter fact implies that the induced Chevalley-Eilenberg differential

$$
d_{L}^{\nabla^{\natural}}: \Gamma\left(\Lambda^{\bullet} L^{\vee} \otimes \hat{S}\left(B^{\vee}\right)\right) \rightarrow \Gamma\left(\Lambda^{\bullet+1} L^{\vee} \otimes \hat{S}\left(B^{\vee}\right)\right)
$$

is a derivation of the algebra $\Gamma\left(\Lambda^{\bullet} L^{\vee} \otimes \hat{S}\left(B^{\vee}\right)\right)$, and can thus be regarded as a homological vector field on the graded manifold $L[1] \oplus B$. One of the main results of [45] is the following theorem.

Theorem 2.13 ([45, Theorem 4.7]). Given a Lie pair $(L, A)$, together with a splitting of the short exact sequence $0 \rightarrow A \rightarrow L \rightarrow B \rightarrow 0$ and a torsion-free $L$-connection on $B$, the dg manifold $\left(L[1] \oplus B, d_{L}^{\nabla^{k}}\right)$ constructed above and the $d g$ manifold $(L[1] \oplus B, Q)$ constructed via the Fedosov iteration in Theorem 2.11 coincide, i.e. $d_{L}^{\nabla^{k}}=Q$.

The Fedosov dg manifolds obtained from different choices of a splitting and a connection are isomorphic to one another. The isomorphism can be made explicit via the associated PBW maps. This shall be needed in Section 3.3, where we will establish the uniqueness claim (2b) of Theorem A from the introduction.

We consider two different choices $j_{1}, \nabla_{1}$ and $j_{2}, \nabla_{2}$ of a splitting $B \rightarrow L$ and a torsion-free $L$-connection on $B$ as before; the two induced homological vector fields $Q_{1}$ and $Q_{2}$ on $L[1] \oplus B$; and the two induced Poincaré-Birkhoff-Witt isomorphisms $\mathrm{pbw}_{1}$ and $\mathrm{pbw}_{2}$. The composition of the latter

$$
\psi:=\mathrm{pbw}_{1}^{-1} \circ \mathrm{pbw}_{2}: \Gamma(S B) \rightarrow \Gamma(S B)
$$

is an automorphism of the $C^{\infty}(M)$-coalgebra $\Gamma(S B)$ intertwining the two induced $L$-module structures. Likewise, the dual map $\psi^{\vee}: \Gamma\left(\hat{S}\left(B^{\vee}\right)\right) \rightarrow \Gamma\left(\hat{S}\left(B^{\vee}\right)\right)$ is an automorphism of the $C^{\infty}(M)$-algebra $\Gamma\left(\hat{S}\left(B^{\vee}\right)\right)$ intertwining the two induced $L$-module structures. Finally, it follows immediately that

$$
\operatorname{id} \otimes \psi^{\vee}:\left(\Gamma\left(\Lambda^{\bullet} L^{\vee} \otimes \hat{S}\left(B^{\vee}\right)\right), Q_{1}\right) \rightarrow\left(\Gamma\left(\Lambda^{\bullet} L^{\vee} \otimes \hat{S}\left(B^{\vee}\right)\right), Q_{2}\right)
$$

is an isomorphism of $\mathrm{dg}$ manifolds $\left(L[1] \oplus B, Q_{2}\right) \rightarrow\left(L[1] \oplus B, Q_{1}\right)$.

2.3. Fedosov dg Lie algebroids. Let $(L, A)$ be a Lie pair over a base manifold $M$. Given a splitting $j: B \rightarrow L$ of the short exact sequence of vector bundles $0 \rightarrow A \rightarrow L \rightarrow B \rightarrow 0$ and a torsion-free $L$-connection $\nabla$ on $B$, one constructs a Fedosov $\operatorname{dg}$ manifold $(\mathcal{M}, Q)$, where $\mathcal{M}=L[1] \oplus B$, as in Theorem 2.11.

Let $R=C^{\infty}(M)$. Let $\mathcal{F} \rightarrow \mathcal{M}$ denote the pullback of the vector bundle $B \rightarrow M$ through the surjective submersion $\mathcal{M} \rightarrow M$. It is a graded vector bundle whose total space $\mathcal{F}$ is the graded manifold with base $M$ associated with the graded vector bundle $L[1] \oplus B \oplus B \rightarrow M$. Its space of sections $\Gamma(\mathcal{F} \rightarrow \mathcal{M})$ is canonically identified with $C^{\infty}(\mathcal{M}) \otimes_{R} \Gamma(B) \cong \Gamma\left(\Lambda^{\bullet} L^{\vee} \otimes \hat{S}\left(B^{\vee}\right) \otimes B\right)$. It is naturally a vector subbundle 
of $T_{\mathcal{M}} \rightarrow \mathcal{M}$; the inclusion $\Gamma(\mathcal{F} \rightarrow \mathcal{M}) \hookrightarrow \mathfrak{X}(\mathcal{M})$ takes the section $\left(\lambda \otimes \chi^{J}\right) \otimes \partial_{k} \in C^{\infty}(\mathcal{M}) \otimes_{R} \Gamma(B)$ of the vector bundle $\mathcal{F} \rightarrow \mathcal{M}$ to the derivation $\mu \otimes \chi^{M} \mapsto \lambda \wedge \mu \otimes M_{k} \chi^{M+J-e_{k}}$ of $C^{\infty}(\mathcal{M})$.

Alternatively, denote by $T_{\text {ver }} B \rightarrow B$ the formal vertical tangent bundle of the vector bundle $B \rightarrow M$, which consists of all formal vertical tangent vectors of $B$. Its space of sections $\mathfrak{X}_{\mathrm{ver}}(B):=\Gamma\left(B ; T_{\mathrm{ver}} B\right)$ is naturally isomorphic to $\Gamma\left(\hat{S}\left(B^{\vee}\right) \otimes B\right)$. Indeed $T_{\text {ver }} B$ is a double vector bundle [32], which is isomorphic to $B \oplus B$. Consider the projection pr: $\mathcal{M}=L[1] \oplus B \rightarrow B$. Then $\mathcal{F}$ is isomorphic to the pull back bundle $\mathrm{pr}^{*} T_{\mathrm{ver}} B$.

Proposition 2.14. The subbundle $\mathcal{F} \subset T_{\mathcal{M}}$ is a dg integrable distribution (or a $d g$ foliation) of the $d g$ manifold $(\mathcal{M}, Q)$, i.e. $\mathcal{F}$ is a dg Lie subalgebroid of the tangent $d g$ Lie algebroid $T_{\mathcal{M}} \rightarrow \mathcal{M}$.

Proof. It is simple to see that $\mathcal{F} \rightarrow \mathcal{M}$ is a Lie subalgebroid of $T_{\mathcal{M}} \rightarrow \mathcal{M}$. Hence it suffices to show that $\mathcal{F}$ admits a dg manifold structure such that $\mathcal{F} \rightarrow \mathcal{M}$ is a dg subbundle of $T_{\mathcal{M}} \rightarrow \mathcal{M}$ - the compatibility condition (12) holds automatically since $T_{\mathcal{M}} \rightarrow \mathcal{M}$ is a dg Lie algebroid (see Example 2.6). According to Remark 2.3, it suffices to prove that $\Gamma(\mathcal{M} ; \mathcal{F})$ is a dg module over $\left(C^{\infty}(\mathcal{M}), Q\right)$. It is clear that $\Gamma(\mathcal{M} ; \mathcal{F}) \cong \Gamma\left(\Lambda^{\bullet} L^{\vee}\right) \otimes_{R} \mathfrak{X}_{\text {ver }}(B)$. From Equation (26), it follows that $\Gamma\left(\Lambda^{\bullet} L^{\vee}\right) \otimes_{R} \mathfrak{X}_{\text {ver }}(B)$ is stable under the Lie derivative $\mathcal{L}_{Q}$. Moreover, we have

$$
\mathcal{L}_{Q}(\xi \cdot(\eta \otimes X))=Q(\xi) \cdot(\eta \otimes X)+(-1)^{|\xi|} \xi \cdot \mathcal{L}_{Q}(\eta \otimes X),
$$

for all homogeneous $\xi \in \Gamma\left(\Lambda^{\bullet} L^{\vee} \otimes \hat{S} B^{\vee}\right), \eta \in \Gamma\left(\Lambda^{\bullet} L^{\vee}\right)$, and $X \in \mathfrak{X}_{\text {ver }}(B)$. Therefore, $\Gamma(\mathcal{M} ; \mathcal{F})$ is a dg module over $\left(C^{\infty}(\mathcal{M}), Q\right)$.

Any dg Lie algebroid constructed in this manner is called a Fedosov dg Lie algebroid associated with the Lie pair $(L, A)$.

Next, we will identify the space of polyvector fields on the Fedosov dg Lie algebroid $\mathcal{F}$ over $\mathcal{M}$.

Set

$$
\mathcal{T}_{\text {poly }}^{k}=\Gamma\left(\Lambda^{k+1} B\right)
$$

and let $\mathscr{T}_{\text {poly }}^{k}$ denote $\Gamma\left(B ; \Lambda^{k+1} T_{\text {ver }} B\right)$, the space of formal vertical $(k+1)$-vector fields on $B$. It is clear that

$$
\mathscr{T}_{\text {poly }}^{k} \cong \Gamma\left(\hat{S}\left(B^{\vee}\right)\right) \otimes_{R} \mathcal{T}_{\text {poly }}^{k}
$$

and

$$
\Gamma\left(\mathcal{M} ; \Lambda^{k+1} \mathcal{F}\right) \cong \Gamma\left(\Lambda^{\bullet} L^{\vee}\right) \otimes_{R} \mathscr{T}_{\text {poly }}^{k} \cong \Gamma\left(\Lambda^{\bullet} L^{\vee} \otimes \hat{S} B^{\vee}\right) \otimes_{R} \mathcal{T}_{\text {poly }}^{k}
$$

Since $\mathcal{F}$ is a dg Lie subalgebroid of $T_{\mathcal{M}}$, the subspace $\Gamma\left(\mathcal{M} ; \Lambda^{k+1} \mathcal{F}\right) \cong \Gamma\left(\Lambda^{\bullet} L^{\vee} \otimes \hat{S}^{\vee}\right) \otimes_{R} \mathcal{T}_{\text {poly }}^{k}$ of the space $T_{\text {poly }}^{k}(\mathcal{M})$ of $(k+1)$-vector fields on $\mathcal{M}=L[1] \oplus B$ is stable under $\mathcal{L}_{Q}$, we obtain a cochain complex

$$
\cdots \longrightarrow \Gamma\left(\Lambda^{u} L^{\vee} \otimes \hat{S} B^{\vee}\right) \otimes_{R} \mathcal{T}_{\text {poly }}^{k} \stackrel{\mathcal{L}_{Q}}{\longrightarrow} \Gamma\left(\Lambda^{u+1} L^{\vee} \otimes \hat{S} B^{\vee}\right) \otimes_{R} \mathcal{T}_{\text {poly }}^{k} \longrightarrow \cdots
$$

for each $k \geqslant-1$.

Applying Proposition 2.7 to the Fedosov dg Lie algebroid $\mathcal{F} \rightarrow \mathcal{M}$, we obtain the following

Proposition 2.15. The total complex $\left(\operatorname{tot}\left(\Gamma\left(\Lambda^{\bullet} L^{\vee} \otimes \hat{S} B^{\vee}\right) \otimes_{R} \mathcal{T}_{\text {poly }}^{\bullet}\right), \mathcal{L}_{Q}\right)$ admits a differential Gerstenhaber algebra, whence a dgla structure.

Finally, we consider the space of polydifferential operators on the Fedosov dg Lie algebroid $\mathcal{F}$ over $\mathcal{M}$. 
Let $\mathscr{D}_{\text {poly }}^{k}$ denote the space of formal vertical $(k+1)$-differential operators on the vector bundle $B$ and set $\mathscr{D}_{\text {poly }}^{\bullet}=\bigoplus_{k=-1}^{\infty} \mathscr{D}_{\text {poly }}^{k}$. There exists a canonical isomorphism

$$
\Gamma(\hat{S}\left(B^{\vee}\right) \otimes \underbrace{S(B) \otimes \cdots \otimes S(B)}_{k+1 \text { factors }}) \stackrel{\varphi}{\cong} \mathscr{D}_{\text {poly }}^{k}
$$

In terms of local dual frames $\left\{\chi_{i}\right\}_{i=1, \ldots, r}$ and $\left\{\partial_{j}\right\}_{j=1, \ldots, r}$ for $B^{\vee}$ and $B$ respectively, and the corresponding local frames $\left\{\chi^{I}\right\}_{I \in \mathbb{N}^{r}}$ and $\left\{\partial_{J}\right\}_{J \in \mathbb{N}^{r}}$ for $\hat{S}\left(B^{\vee}\right)$ and $S(B)$ respectively, the isomorphism $\varphi$ sends $\chi^{I} \otimes \partial_{J_{0}} \otimes \cdots \otimes \partial_{J_{k}} \in \Gamma(\hat{S}\left(B^{\vee}\right) \otimes \underbrace{S(B) \otimes \cdots \otimes S(B)}_{k+1 \text { factors }})$ to the $(k+1)$-differential operator

$$
\Gamma\left(\hat{S}\left(B^{\vee}\right)\right)^{\otimes k+1} \ni \chi^{I_{0}} \otimes \cdots \otimes \chi^{I_{k}} \longmapsto \chi^{I} \cdot \partial_{J_{0}}\left(\chi^{I_{0}}\right) \cdots \partial_{J_{k}}\left(\chi^{I_{k}}\right) \in \Gamma\left(\hat{S}\left(B^{\vee}\right)\right) .
$$

The algebra $C^{\infty}(L[1] \oplus B)$ is a module over its subalgebra $\Gamma\left(\Lambda^{\bullet} L\right) \cong \Gamma\left(\Lambda^{\bullet} L^{\vee} \otimes S^{0}\left(B^{\vee}\right)\right)$. The subspace of $D_{\text {poly }}^{\bullet}(L[1] \oplus B)$ comprised of all $\Gamma\left(\Lambda^{\bullet} L^{\vee}\right)$-multilinear polydifferential operators is easily identified to tot $\left(\Gamma\left(\Lambda^{\bullet} L^{\vee}\right) \otimes_{R} \mathscr{D}_{\text {poly }}^{\bullet}\right)$. It is simple to see that the universal enveloping algebra $\mathcal{U}(\mathcal{F})$ of the dg Lie algebroid $\mathcal{F} \rightarrow \mathcal{M}$ is naturally identified with $\Gamma\left(\Lambda^{\bullet} L^{\vee}\right) \otimes_{R} \mathscr{D}_{\text {poly }}^{0}$, which is a dg Hopf algebroid over $\mathcal{R}=C^{\infty}(\mathcal{M}) \cong \Gamma\left(\Lambda^{\bullet} L^{\vee} \otimes \hat{S} B^{\vee}\right)$. Moreover, $\mathcal{U}(\mathcal{F})$ is a dg Hopf subalgebroid of $D_{\text {poly }}^{0}(L[1] \oplus B)$. Notice that

$$
s \mathcal{U}(\mathcal{F})^{\otimes k+1} \cong \Gamma\left(\Lambda^{\bullet} L^{\vee}\right) \otimes_{R} \mathscr{D}_{\text {poly }}^{k}
$$

Since $\mathcal{F}$ is a dg Lie subalgebroid of $T_{\mathcal{M}}$, the subspace

$$
\operatorname{tot}_{\oplus} \operatorname{sU}(\mathcal{F})^{\bullet+1} \cong \operatorname{tot}\left(\Gamma\left(\Lambda^{\bullet} L^{\vee}\right) \otimes_{R} \mathscr{D}_{\text {poly }}^{\bullet}\right)
$$

of $D_{\text {poly }}^{\bullet}(\mathcal{M})$ is stable under the Hochschild coboundary operator $\llbracket Q+m,-\rrbracket$. Here $m=1 \otimes 1$ is the element of $s \mathcal{U}(\mathcal{F})^{\otimes 2}$ arising from the multiplication of $C^{\infty}(\mathcal{M})$.

The Lie bracket (19) and the cup product $(22)$ on $\operatorname{tot}_{\oplus} s \mathcal{U}(\mathcal{F})^{\bullet+1}$ carry over to a $\Gamma\left(\Lambda^{\bullet} L^{\vee}\right)$-linear Lie bracket and cup product on tot $\left(\Gamma\left(\Lambda^{\bullet} L^{\vee}\right) \otimes_{R} \mathscr{D}_{\text {poly }}^{\bullet}\right)$ through the identification (33).

Applying Proposition 2.8 to the Fedosov dg Lie algebroid $\mathcal{F} \rightarrow \mathcal{M}$, we obtain the following

\section{Proposition 2.16.}

(1) The triple ( $\left.\operatorname{tot}\left(\Gamma\left(\Lambda^{\bullet} L^{\vee}\right) \otimes_{R} \mathscr{D}_{\text {poly }}\right), \llbracket Q+m,-\rrbracket, \llbracket-,-\rrbracket\right)$ is a dgla.

(2) The cohomology group $\mathbb{H}^{\bullet}\left(\operatorname{tot}\left(\Gamma\left(\Lambda^{\bullet} L^{\vee}\right) \otimes_{R} \mathscr{D} \bullet\right.\right.$ poly $\left.), \llbracket Q+m,-\rrbracket\right)$ equipped with the induced Lie bracket and cup product is a Gerstenhaber algebra.

\section{3. $L_{\infty}$ Algebra structures}

In this section, we endow the spaces of polyvector fields and polydifferential operators of a Lie pair see Section 1 - with $L_{\infty}$ algebra structures, which are canonical up to $L_{\infty}$ isomorphism.

3.1. Dolgushev-Fedosov contraction and $L_{\infty}$ algebra structure on the space of polyvector fields of a Lie pair. The following lemma is straightforward.

Lemma 3.1. The subspace $\Gamma\left(\Lambda^{\bullet} L^{\vee}\right) \otimes_{R} \mathscr{T}_{\text {poly }}^{k}$ of the space $T_{\text {poly }}^{k}(L[1] \oplus B)$ of $(k+1)$-vector fields on $L[1] \oplus B$ is stable under $\mathcal{L}_{\delta}$. 
Also, note that the following diagram commutes:

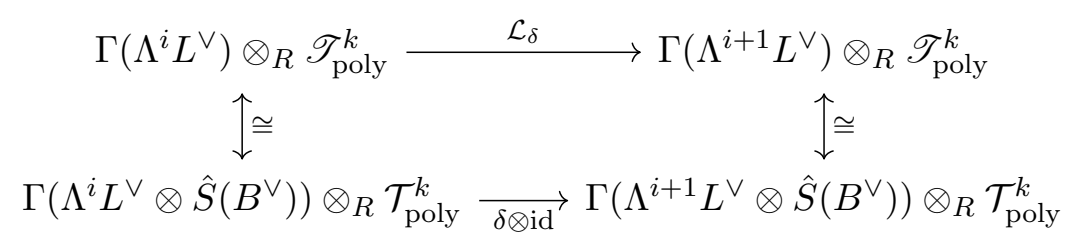

Since the vector field $\delta$ on $L[1] \oplus B$ is homological, we obtain the cochain complex

$$
\cdots \longrightarrow \Gamma\left(\Lambda^{i} L^{\vee}\right) \otimes_{R} \underset{\text { poly }}{k} \stackrel{\mathcal{L}_{-\delta}}{\longrightarrow} \Gamma\left(\Lambda^{i+1} L^{\vee}\right) \otimes_{R} \underset{\text { poly }}{\mathscr{T}^{k} \longrightarrow \cdots}
$$

which admits the descending filtration

$$
\mathscr{F}_{m}=\bigoplus_{i=0}^{\operatorname{rk}(L)} \Gamma\left(\Lambda^{i} L^{\vee} \otimes \hat{S}^{\geqslant m-i} B^{\vee}\right) \otimes_{R} \mathcal{T}_{\text {poly }}^{k} .
$$

We shall denote by $\tau_{\natural}, \sigma_{\natural}$ and $h_{\natural}$ the maps defined by the following commutative diagrams (where $\tau$, $\sigma$, and $h$ are the maps introduced in Section 2.2)

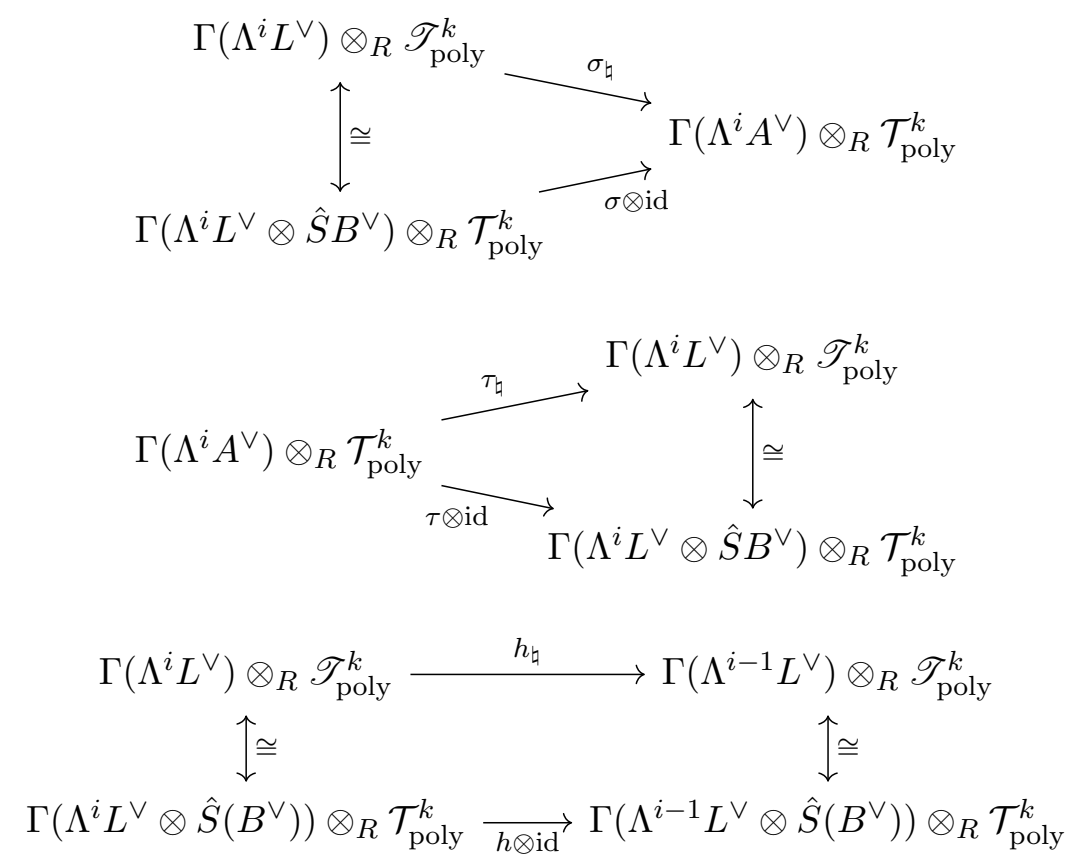

Adapting the proof of [45, Proposition 4.3], we obtain

Proposition 3.2. The complex $\left(\Gamma\left(\Lambda^{\bullet} L^{\vee}\right) \otimes_{R} \mathscr{T}_{\text {poly }}^{k}, \mathcal{L}_{-\delta}\right)$ contracts onto $\left(\Gamma\left(\Lambda^{\bullet} A^{\vee}\right) \otimes_{R} \mathcal{T}_{\text {poly }}^{k}, 0\right)$. More precisely, we have the filtered contraction

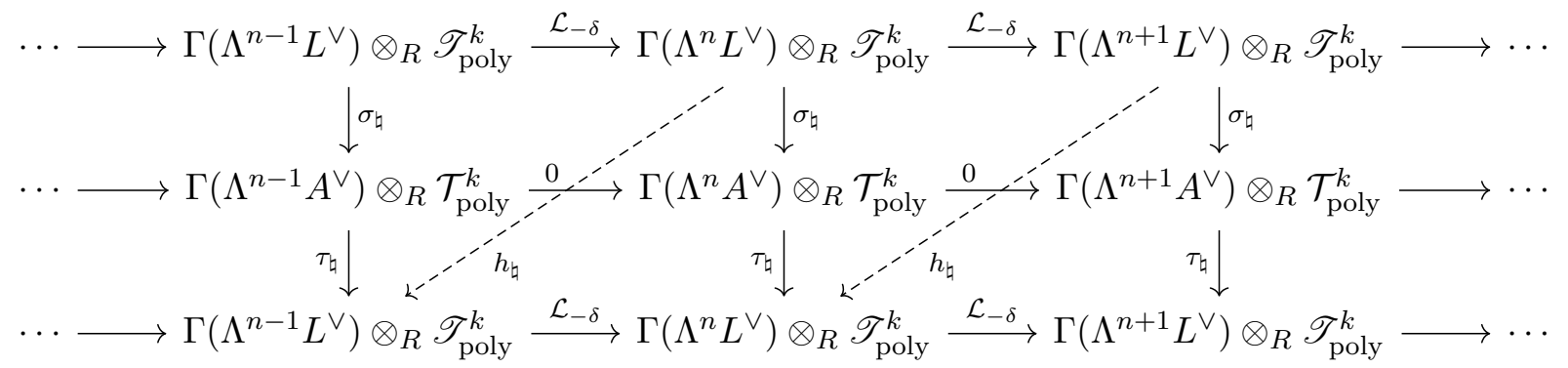

where $\tau_{\natural}, \sigma_{\natural}$ and $h_{\natural}$ are defined by the above commutative diagrams (34)-(35). 
Lemma 3.3. The contraction $\left(\tau_{\natural}, \sigma_{\natural}, h_{\natural}\right)$ in Proposition 3.2 is a semifull algebra contraction - on both sides, the associative multiplication is the wedge product. Moreover, the maps $\tau_{\natural}$ and $\sigma_{\natural}$ preserve the wedge products.

Proof. This follows immediately from the definitions and the corresponding statements for $(\tau, \sigma, h)$ see Lemma 2.10.

Consider the homological vector field $Q$ on $L[1] \oplus B$ introduced in Theorem 2.11:

$$
Q=-\delta+\rho \quad \text { with } \quad \rho=d_{L}^{\nabla}+X^{\nabla} \quad \text { and } \quad X^{\nabla} \in \Gamma\left(L^{\vee} \otimes \hat{S}^{\geqslant 2}\left(B^{\vee}\right) \otimes B\right) .
$$

Proposition 3.4. There exists a contraction

$$
\left(\operatorname{tot}\left(\Gamma\left(\Lambda^{\bullet} A^{\vee}\right) \otimes_{R} \mathcal{T}_{\text {poly }}^{\bullet}\right), d_{A}^{\text {Bott }}\right) \underset{\sigma_{\natural}}{\stackrel{\breve{\tau}_{\natural}}{\rightleftarrows}}\left(\operatorname{tot}\left(\Gamma\left(\Lambda^{\bullet} L^{\vee}\right) \otimes_{R} \mathscr{T}_{\text {poly }}^{\bullet}\right), \mathcal{L}_{Q}\right) \circlearrowright \breve{h}_{\natural}
$$

More precisely, for every $k \geqslant-1$, we have the (filtered) contraction

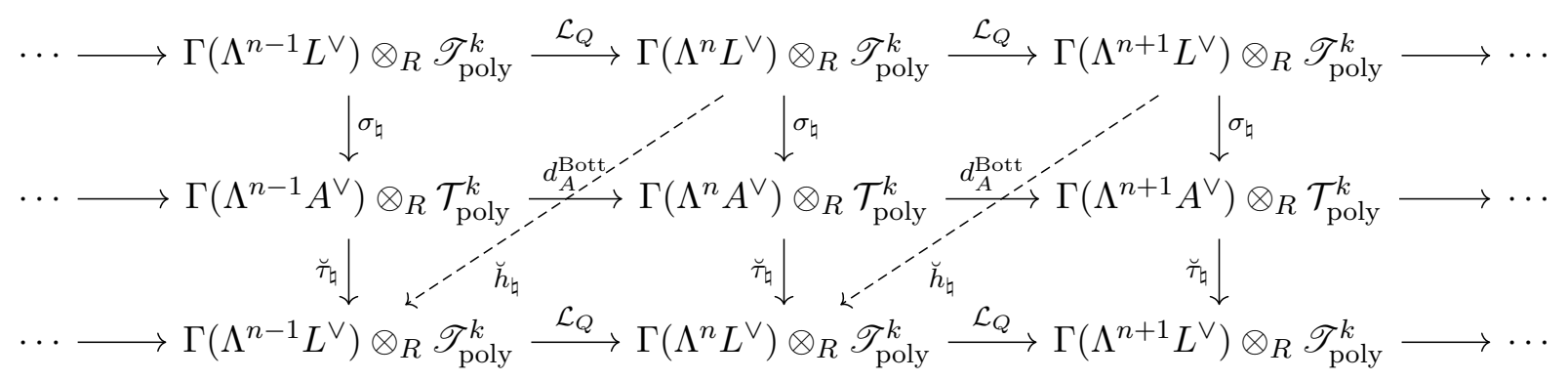

where

$$
\breve{h}_{\natural}=\sum_{l=0}^{\infty}\left(h_{\natural} \mathcal{L}_{\rho}\right)^{l} h_{\natural}, \quad \text { and } \quad \breve{\tau}_{\natural}=\sum_{l=0}^{\infty}\left(h_{\natural} \mathcal{L}_{\rho}\right)^{l} \tau_{\natural} .
$$

Moreover, the cochain maps $\breve{\tau}_{\natural}$ and $\sigma_{\natural}$ intertwine the wedge products on their domain and codomain.

As an immediate consequence of Proposition 3.4, by considering the bigradings on both sides of (36), we obtain the following

Corollary 3.5. For every $k \geqslant-1$, we have a contraction

$$
\left.\left.\left(\Gamma\left(\Lambda^{\bullet} A^{\vee}\right) \otimes_{R} \mathcal{T}_{\text {poly }}^{k}\right), d_{A}^{\text {Bott }}\right) \underset{\sigma_{\natural}}{\stackrel{\breve{\tau_{\natural}}}{\rightleftarrows}}\left(\Gamma\left(\Lambda^{\bullet} L^{\vee}\right) \otimes_{R} \mathscr{T}_{\text {poly }}^{k}\right), \mathcal{L}_{Q}\right) \breve{h}_{\natural}
$$

The case $k=-1$ was established in [45, Proposition 5.4].

The proof of Proposition 3.4 requires the following technical results.

Lemma 3.6. Let $\mathrm{pr}_{0}$ denote the canonical projection $\hat{S}\left(B^{\vee}\right) \otimes B \rightarrow S^{0}\left(B^{\vee}\right) \otimes B$. For all $a \in \Gamma(A)$ and $j \in\{1, \ldots, r\}$, we have

$$
\operatorname{pr}_{0}\left(\left[\nabla_{a}^{\xi}, \partial_{j}\right]\right)=\nabla_{a}^{\mathrm{Bott}}\left(\partial_{j}\right) .
$$

Recall that $\left\{\partial_{j}\right\}_{j=1, \ldots, r}$ is a local frame for the vector subbundle $B \cong S^{0}\left(B^{\vee}\right) \otimes B$ of $\hat{S}\left(B^{\vee}\right) \otimes B$. Here we think of $\partial_{j}$ as a local section of $\hat{S}\left(B^{\vee}\right) \otimes B$. The sections of the vector bundle $\hat{S}\left(B^{\vee}\right) \otimes B$ may be interpreted as fiberwise formal vertical vector fields on $B$ - they act as derivations of the algebra $\Gamma\left(\hat{S}\left(B^{\vee}\right)\right)$ of fiberwise formal functions on $B$ in a natural fashion. 
Proof. We have seen that, for all $a \in \Gamma(A)$, the operator $\nabla_{a}^{\xi}$ is a derivation of $\Gamma\left(\hat{S}\left(B^{\vee}\right)\right)$, which stabilizes the filtration $\Gamma\left(\hat{S}^{\geqslant n}\left(B^{\vee}\right)\right)$. Therefore, there exist local sections $\theta_{k}^{M}$ of $A^{\vee}$ such that

$$
\nabla_{a}^{\xi} \chi_{k}=\sum_{\substack{M \in \mathbb{N}_{0}^{r} \\|M| \geqslant 1}} \iota_{a} \theta_{k}^{M} \cdot \chi^{M} .
$$

It follows that $\nabla_{a}^{\xi}$ may be regarded as a section of $\hat{S}^{\geqslant 1}\left(B^{\vee}\right) \otimes B$ :

$$
\nabla_{a}^{\xi}=\sum_{k=1}^{r}\left(\sum_{\substack{M \in \mathbb{N}_{0}^{r} \\|M| \geqslant 1}} \iota_{a} \theta_{k}^{M} \cdot \chi^{M}\right) \partial_{k} .
$$

On one hand, it follows from

$$
\begin{array}{r}
{\left[\nabla_{a}^{\xi}, \partial_{j}\right]=\nabla_{a}^{\xi} \circ \partial_{j}-\partial_{j} \circ \nabla_{a}^{\xi}=\sum_{k=1}^{r} \sum_{|M| \geqslant 1} \iota_{a} \theta_{k}^{M} \cdot \chi^{M} \partial_{k} \circ \partial_{j}-\sum_{k=1}^{r} \sum_{|M| \geqslant 1} \iota_{a} \theta_{k}^{M} \cdot \partial_{j} \circ\left(\chi^{M} \partial_{k}\right)} \\
=-\sum_{k=1}^{r} \sum_{|M| \geqslant 1} \iota_{a} \theta_{k}^{M} \cdot M_{j} \chi^{M-e_{j}} \cdot \partial_{k}
\end{array}
$$

that

$$
\operatorname{pr}_{0}\left(\left[\nabla_{a}^{\xi}, \partial_{j}\right]\right)=-\sum_{k=1}^{r} \iota_{a} \theta_{k}^{e_{j}} \cdot \partial_{k} .
$$

On the other hand, it follows from

$$
0=\varrho_{a} \underbrace{\left\langle\chi_{k} \mid \partial_{j}\right\rangle}_{\delta_{k, j}}=\left\langle\nabla_{a}^{\xi} \chi_{k} \mid \partial_{j}\right\rangle+\left\langle\chi_{k} \mid \nabla_{a}^{\xi} \partial_{j}\right\rangle
$$

and the fact that $\nabla_{a}^{\xi}$ stabilizes the subspace $\Gamma\left(S^{1}(B)\right)$ of $\Gamma(S(B))$ that

$$
\begin{aligned}
\nabla_{a}^{\xi}\left(\partial_{j}\right) & =\sum_{k}\left\langle\chi_{k} \mid \nabla_{a}^{\xi} \partial_{j}\right\rangle \partial_{k} \\
& =-\sum_{k}\left\langle\nabla_{a}^{\xi} \chi_{k} \mid \partial_{j}\right\rangle \partial_{k} \\
& =-\sum_{k} \sum_{|M| \geqslant 1} \iota_{a} \theta_{k}^{M} \cdot\left\langle\chi^{M} \mid \partial_{j}\right\rangle \cdot \partial_{k} \\
& =-\sum_{k} \iota_{a} \theta_{k}^{e_{j}} \cdot \partial_{k} .
\end{aligned}
$$

Finally, for all $a \in \Gamma(A)$ and $b \in \Gamma(B)$, we have $\nabla_{a}^{\xi}(b)=\nabla_{a}^{\text {Bott }}(b)$ as

$$
\begin{aligned}
\operatorname{pbw}\left(\nabla_{a}^{\xi} b-\nabla_{a}^{\mathrm{Bott}} b\right)=a \cdot \operatorname{pbw}(b)-\operatorname{pbw}(q[a, j(b)]) & \\
& =a \cdot j(b)-j \circ q([a, j(b)])=j(b) \cdot a+\underbrace{p([a, j(b)])}_{\in \Gamma(A)}=0
\end{aligned}
$$

in $\frac{\mathcal{U}(L)}{\mathcal{U}(L) \Gamma(A)}$. The proof is complete.

Lemma 3.7. $\sigma_{\natural} \circ \mathcal{L}_{\rho} \circ \tau_{\natural}=d_{A}^{\text {Bott }}$ 
Proof. Let $\left(l_{k}\right)_{k \in\{1, \ldots, \mathrm{rk} L\}}$ denote any local frame of $L$ and let $\left(\lambda_{k}\right)_{k \in\{1, \ldots, \mathrm{rk} L\}}$ denote the dual local frame of $L^{\vee}$. Likewise, let $\left(a_{k}\right)_{k \in\{1, \ldots, \text { rk } A\}}$ denote any local frame of $A$ and let $\left(\alpha_{k}\right)_{k \in\{1, \ldots, \text { rk } A\}}$ denote the dual local frame of $A^{\vee}$. For all $\omega \in \Gamma\left(\Lambda^{\bullet} A^{\vee}\right), n \in \mathbb{N}$, and $j_{0}, \ldots, j_{n} \in\{1, \ldots, r\}$, we have

$$
\begin{aligned}
& \sigma_{\natural}\left(\left[\rho, \tau_{\natural}\left(\omega \otimes \partial_{j_{0}} \wedge \cdots \wedge \partial_{j_{n}}\right)\right]\right) \\
=\sigma_{\natural}( & {\left.\left.\left[\rho, p^{\top} \omega \otimes 1 \otimes \partial_{j_{0}} \wedge \cdots \wedge \partial_{j_{n}}\right)\right]\right) } \\
=\sigma_{\natural}( & \left.d_{L}\left(p^{\top} \omega\right) \otimes 1 \otimes \partial_{j_{0}} \wedge \cdots \wedge \partial_{j_{n}}\right) \\
& \left.\quad+\sum_{k} \lambda_{k} \wedge p^{\top} \omega \otimes\left[\nabla_{l_{k}}-\iota_{l_{k}} \Xi, 1 \otimes \partial_{j_{0}} \wedge \cdots \wedge \partial_{j_{n}}\right]\right) \\
=\sigma\left(d_{L}\left(p^{\top} \omega\right) \otimes 1\right) \otimes \partial_{j_{0}} \wedge \cdots \wedge \partial_{j_{n}} & \quad+\sum_{k} \sigma_{\natural}\left(p^{\top} \alpha_{k} \wedge p^{\top} \omega \otimes\left[\nabla_{a_{k}}^{\xi}, 1 \otimes \partial_{j_{0}} \wedge \cdots \wedge \partial_{j_{n}}\right]\right) \\
=d_{A} \omega \otimes \partial_{j_{0}} \wedge \cdots \wedge \partial_{j_{n}} & \quad+\sum_{k} \sigma_{\natural}\left(p^{\top}\left(\alpha_{k} \wedge \omega\right) \otimes\left\{\sum_{t=0}^{n} 1 \otimes \partial_{j_{0}} \wedge \cdots \wedge\left[\nabla_{a_{k}}^{\xi}, \partial_{j_{t}}\right] \wedge \cdots \wedge \partial_{j_{n}}\right\}\right) \\
=d_{A} \omega & \otimes \partial_{j_{0}} \wedge \cdots \wedge \partial_{j_{n}} \\
& \quad+\sum_{k} \sum_{t=0}^{n} \alpha_{k} \wedge \omega \otimes \partial_{j_{0}} \wedge \cdots \wedge \operatorname{pr}_{0}\left[\nabla_{a_{k}}^{\xi}, \partial_{j_{t}}\right] \wedge \cdots \wedge \partial_{j_{n}} .
\end{aligned}
$$

It follows from Lemma 3.6 that

$$
\operatorname{pr}_{0}\left[\nabla_{a_{k}}^{\xi}, \partial_{j_{t}}\right]=\nabla_{a_{k}}^{\xi}\left(\partial_{j_{t}}\right)=\nabla_{a_{k}}^{\text {Bott }}\left(\partial_{j_{t}}\right) .
$$

Hence, we conclude that $\sigma_{\natural} \circ \mathcal{L}_{\rho} \circ \tau_{\natural}=d_{A}^{\text {Bott }}$.

Proof of Proposition 3.4. We proceed by homological perturbation - see Lemma A.1 and also [45, Lemma A.1] and [21, §1]. Starting from the filtered contraction of Proposition 3.2, it suffices to perturb the coboundary operator $\mathcal{L}_{-\delta}$ by the operator $\mathcal{L}_{\rho}$. One checks that $\sigma_{\natural} \mathcal{L}_{\rho} h_{\natural}=0$. It follows that

$$
\breve{\sigma}_{\natural}:=\sum_{l=0}^{\infty} \sigma_{\natural}\left(\mathcal{L}_{\rho} h_{\natural}\right)^{l}=\sigma_{\natural}
$$

and, making use of Lemma 3.7,

$$
\vartheta:=\sum_{l=0}^{\infty} \sigma_{\natural}\left(\mathcal{L}_{\rho} h_{\natural}\right)^{l} \mathcal{L}_{\rho} \tau_{\natural}=\sigma_{\natural} \mathcal{L}_{\rho} \tau_{\natural}=d_{A}^{\text {Bott }} .
$$

The result follows immediately since $-\delta+\rho=Q$.

Finally, the claim that $\sigma_{\natural}$ is compatible with the wedge products is contained in Lemma 3.3, while the same statement for $\breve{\tau}_{\natural}$ follows from Lemmas 3.3, A.3 and A.4.

The next proposition gives an alternative characterization of the map $\breve{\tau}_{\natural}$ as the solution of an initial value problem.

Proposition 3.8. Given $x \in \Gamma\left(\Lambda^{\bullet} A^{\vee}\right) \otimes_{R} \mathcal{T}_{\text {poly }}^{\diamond}$ and $y \in \Gamma\left(\Lambda^{\bullet} L^{\vee}\right) \otimes_{R} \mathscr{T}_{\text {poly }}^{\diamond}$, we have

$$
\breve{\tau}_{\natural}(x)=y \quad \text { if and only if } \quad\left\{\begin{array} { l } 
{ h _ { \natural } ( y ) = 0 } \\
{ h _ { \natural } \mathcal { L } _ { Q } ( y ) = 0 } \\
{ \sigma _ { \natural } ( y ) = x }
\end{array} \quad \text { if and only if } \quad \left\{\begin{array}{l}
\mathcal{L}_{\eta}(y)=0 \\
\mathcal{L}_{[\eta, Q]}(y)=0 \\
\sigma_{\natural}(y)=x
\end{array}\right.\right.
$$


The derivation $\eta$ was defined in Equation (25).

Proof. Assume $\breve{\tau}_{\natural}(x)=y$. From $h_{\natural} \tau_{\natural}=0$ and $h_{\natural} h_{\natural}=0$, we get

$$
h_{\natural} \breve{\tau}_{\natural}=h_{\natural} \sum_{l=0}^{\infty}\left(h_{\natural} \mathcal{L}_{\rho}\right)^{l} \tau_{\natural}=h_{\natural} \tau_{\natural}+h_{\natural}\left(h_{\natural} \mathcal{L}_{\rho}\right) \sum_{l=0}^{\infty}\left(h_{\natural} \mathcal{L}_{\rho}\right)^{l} \tau_{\natural}=0 .
$$

It follows that

$$
h_{\natural}(y)=h_{\natural} \breve{t}_{\natural}(x)=0 \quad \text { and } \quad \sigma_{\natural}(y)=\sigma_{\natural} \breve{T}_{\natural}(x)=x .
$$

Furthermore, since $\breve{\tau}_{\natural}$ is a chain map, we have

$$
h_{\natural} \mathcal{L}_{Q}(y)=h_{\natural} \mathcal{L}_{Q} \breve{\tau}_{\natural}(x)=h_{\natural} \breve{\tau}_{\natural} d_{A}^{\text {Bott }}(x)=0 .
$$

Conversely, assuming $h_{\natural}(y)=0 ; h_{\natural} \mathcal{L}_{Q}(y)=0$; and $\sigma_{\natural}(y)=x$, it follows from

$$
\breve{\tau}_{\natural} \sigma_{\natural}-\mathrm{id}=\breve{h}_{\natural} \mathcal{L}_{Q}+\mathcal{L}_{Q} \breve{h}_{\natural}
$$

that

$$
\breve{\tau}_{\natural}(x)-y=\breve{\tau}_{\natural} \sigma_{\natural}(y)-y=\breve{h}_{\natural} \mathcal{L}_{Q}(y)+\mathcal{L}_{Q} \breve{h}_{\natural}(y)=\sum_{l=0}^{\infty}\left(h_{\natural} \mathcal{L}_{\rho}\right)^{l} h_{\natural} \mathcal{L}_{Q}(y)+\mathcal{L}_{Q} \sum_{l=0}^{\infty}\left(h_{\natural} \mathcal{L}_{\rho}\right)^{l} h_{\natural}(y)=0
$$

and we can conclude that $\breve{\tau}_{\natural}(x)=y$.

Finally, it is not difficult to show that $\operatorname{ker}\left(h_{\natural}\right)=\operatorname{ker}\left(\mathcal{L}_{\eta}\right)$. It follows that

$$
\left\{\begin{array} { l } 
{ h _ { \natural } ( y ) = 0 } \\
{ h _ { \natural } \mathcal { L } _ { Q } ( y ) = 0 }
\end{array} \quad \text { if and only if } \quad \left\{\begin{array} { l } 
{ \mathcal { L } _ { \eta } ( y ) = 0 } \\
{ \mathcal { L } _ { \eta } \mathcal { L } _ { Q } ( y ) = 0 }
\end{array} \quad \text { if and only if } \quad \left\{\begin{array}{l}
\mathcal{L}_{\eta}(y)=0 \\
\mathcal{L}_{[\eta, Q]}(y)=0
\end{array}\right.\right.\right.
$$

It follows from the homotopy transfer theorem for $L_{\infty}$ algebras $[5,22,17,1,16,6,14,15]$ applied to the contraction in Proposition 3.4 that the dgla structure carried by $\operatorname{tot}\left(\Gamma\left(\Lambda^{\bullet} L^{\vee}\right) \otimes_{R} \mathscr{T}_{\text {poly }}^{\bullet}\right)$ induces an $L_{\infty}$ algebra structure on tot $\left(\Gamma\left(\Lambda^{\bullet} A^{\vee}\right) \otimes_{R} \mathcal{T}_{\text {poly }}^{\bullet}\right)$. Moreover, since the retraction $\sigma_{\natural}$ preserves the wedge products according to Proposition 3.4, we immediately obtain the following

Proposition 3.9. Given a Lie pair $(L, A)$, each choice of a splitting $j: B \rightarrow L$ of the short exact sequence of vector bundles $0 \rightarrow A \rightarrow L \rightarrow B \rightarrow 0$ and of a torsion-free $L$-connection $\nabla$ on $B$ determines

(1) an $L_{\infty}$ algebra structure on tot $\left(\Gamma\left(\Lambda^{\bullet} A^{\vee}\right) \otimes_{R} \mathcal{T}_{\text {poly }}^{\bullet}\right)$ with the operator $d_{A}^{\text {Bott }}$ as unary bracket

(2) and a Gerstenhaber algebra structure on $\mathbb{H}_{\mathrm{CE}}^{\bullet}\left(A, \mathcal{T}_{\text {poly }}^{\bullet}\right)$, the cohomology of the total complex

$$
\left(\operatorname{tot}\left(\Gamma\left(\Lambda^{\bullet} A^{\vee}\right) \otimes_{R} \mathcal{T}_{\text {poly }}^{\bullet}\right), d_{A}^{\text {Bott }}\right)
$$

where the Lie bracket is induced by the binary bracket of the $L_{\infty}$ algebra structure on tot $\left(\Gamma\left(\Lambda^{\bullet} A^{\vee}\right) \otimes_{R} \mathcal{T}_{\text {poly }}^{\bullet}\right)$ and the multiplication by the wedge product (9).

Remark 3.10. One can prove that the $L_{\infty}$ algebra structure on tot $\left(\Gamma\left(\Lambda^{\bullet} A^{\vee}\right) \otimes_{R} \mathcal{T}_{\text {poly }}\right)$ is compatible with the wedge product in the sense that all $L_{\infty}$ multibrackets are multi-derivations with respect to the wedge product. In other words, in the terminology of [2], tot $\left(\Gamma\left(\Lambda^{\bullet} A^{\vee}\right) \otimes_{R} \mathcal{T}_{\text {poly }}^{\bullet}\right)$ is a $(+1)$-shifted derived Poisson algebra. ${ }^{3}$

\footnotetext{
${ }^{3}$ In the context of $\mathbb{Z}_{2}$-grading, (+1)-shifted derived Poisson algebras are also called homotopy Schouten algebras by Khudaverdian-Voronov [24]. Note that, 0-shifted derived Poisson algebras were studied by Oh-Park [41] and CattaneoFelder [8], who called them $P_{\infty}$ algebras.
} 
3.2. Dolgushev-Fedosov contraction and $L_{\infty}$ algebra structure on the space of polydifferential operators of a Lie pair. Denote the space of polydifferential operators on $L[1] \oplus B$ by $D_{\text {poly }}^{\bullet}(L[1] \oplus B)$. The Hochschild cohomology of the Fedosov dg manifold $(L[1] \oplus B, Q)$ is the cohomology of the cochain complex $\left(D_{\text {poly }}^{\bullet}(L[1] \oplus B), \llbracket Q+m,-\rrbracket\right)$. The algebra of functions $C^{\infty}(L[1] \oplus B)$ is a module over its subalgebra $\Gamma\left(\Lambda^{\bullet} L^{\vee}\right) \cong \Gamma\left(\Lambda^{\bullet} L^{\vee} \otimes S^{0}\left(B^{\vee}\right)\right)$. The subspace of $D_{\text {poly }}^{\bullet}(L[1] \oplus B)$ comprised of all $\Gamma\left(\Lambda^{\bullet} L^{\vee}\right)$-multilinear polydifferential operators is easily identified to tot $\left(\Gamma\left(\Lambda^{\bullet} L^{\vee}\right) \otimes_{R} \mathscr{D} \bullet_{\text {poly }}\right)$, the space of polydifferential operators on the Fedosov dg Lie algebroid $\mathcal{F}$. Since $\mathcal{F}$ is a dg Lie subalgebroid of the tangent bundle $T_{\mathcal{M}} \rightarrow \mathcal{M}$ of the Fedosov dg manifold $(L[1] \oplus B, Q)$, it follows that the subspace tot $\left(\Gamma\left(\Lambda^{\bullet} L^{\vee}\right) \otimes_{R} \mathscr{D}_{\text {poly }}^{\bullet}\right)$ of $D_{\text {poly }}^{\bullet}(L[1] \oplus B)$ is stable under the Hochschild coboundary operator $\llbracket Q+m,-\rrbracket$ of the Fedosov dg manifold $(L[1] \oplus B, Q)$.

We also have the following

Lemma 3.11. The subspace tot $\left(\Gamma\left(\Lambda^{\bullet} L^{\vee}\right) \otimes_{R} \mathscr{D}_{\text {poly }}^{\bullet}\right)$ of $D_{\text {poly }}^{\bullet}(L[1] \oplus B)$ is stable under $\llbracket \delta,-\rrbracket$.

Lemma 3.12. The diagram

$$
\begin{aligned}
& \Gamma\left(\Lambda^{p} L^{\vee}\right) \otimes_{R} \mathscr{D}_{\text {poly }}^{v} \stackrel{\llbracket \delta,-\rrbracket}{\longrightarrow} \Gamma\left(\Lambda^{p+1} L^{\vee}\right) \otimes_{R} \mathscr{D}_{\text {poly }}^{v} \\
& (-1)^{p} \llbracket m,-\rrbracket \uparrow \\
& \Gamma\left(\Lambda^{p} L^{\vee}\right) \otimes_{R} \mathscr{D}_{\text {poly }}^{v-1} \stackrel{\llbracket \delta,-\rrbracket}{\longrightarrow} \Gamma\left(\Lambda^{p+1} \llbracket m,-\rrbracket\right. \\
&
\end{aligned}
$$

commutes.

Proof. It suffices to verify that the diagrams

$$
\begin{gathered}
\Gamma\left(\Lambda^{p} L^{\vee}\right) \otimes_{R} \mathscr{D}_{\text {poly }}^{v} \longrightarrow \Gamma\left(\Lambda^{p+1} L^{\vee}\right) \otimes_{R} \mathscr{D}_{\text {poly }}^{v} \\
\operatorname{id} \otimes \varphi \uparrow \cong \\
\operatorname{id} \otimes \varphi \uparrow \cong \\
\Gamma\left(\Lambda^{p} L^{\vee} \otimes \hat{S}\left(B^{\vee}\right)\right) \otimes_{R} \Gamma\left((S B)^{\otimes v+1}\right) \stackrel{\delta \otimes \text { id }}{\longrightarrow} \Gamma\left(\Lambda^{p+1} L^{\vee} \otimes \hat{S}\left(B^{\vee}\right)\right) \otimes_{R} \Gamma\left((S B)^{\otimes v+1}\right)
\end{gathered}
$$

and

$$
\begin{gathered}
\Gamma\left(\Lambda^{p} L^{\vee}\right) \otimes_{R} \mathscr{D}_{\text {poly }}^{v-1} \longrightarrow \Gamma\left(\Lambda^{p} L^{\vee}\right) \otimes_{R} \mathscr{D}_{\text {poly }}^{v} \\
\operatorname{id} \otimes \varphi \uparrow \cong \\
\operatorname{id} \otimes \varphi \uparrow \cong \\
\Gamma\left(\Lambda^{p} L^{\vee} \otimes \hat{S}\left(B^{\vee}\right)\right) \otimes_{R} \Gamma\left((S B)^{\otimes v}\right) \stackrel{\text { id } \otimes(-1)^{v-1} d_{\mathscr{C}}}{\longrightarrow} \Gamma\left(\Lambda^{p} L^{\vee} \otimes \hat{S}\left(B^{\vee}\right)\right) \otimes_{R} \Gamma\left((S B)^{\otimes v+1}\right)
\end{gathered}
$$

commute.

Proposition 3.13. The diagram

$$
\begin{aligned}
& \begin{array}{rrr}
\vdots & \vdots & \vdots \\
\llbracket m,-\rrbracket \uparrow & -\llbracket m,-\rrbracket \uparrow & \llbracket m,-\rrbracket \uparrow
\end{array} \\
& \Gamma\left(\Lambda^{0} L^{\vee}\right) \otimes_{R} \mathscr{D}_{\text {poly }}^{1} \stackrel{\llbracket-\delta,-\rrbracket}{\longrightarrow} \Gamma\left(\Lambda^{1} L^{\vee}\right) \otimes_{R} \mathscr{D}_{\text {poly }}^{1} \stackrel{\llbracket-\delta,-\rrbracket}{\longrightarrow} \Gamma\left(\Lambda^{2} L^{\vee}\right) \otimes_{R} \mathscr{D}_{\text {poly }}^{1} \stackrel{\llbracket-\delta,-\rrbracket}{\longrightarrow} \cdots \\
& \llbracket m,-\rrbracket \uparrow \quad-\llbracket m,-\rrbracket \uparrow \quad \llbracket m,-\rrbracket \uparrow \\
& \Gamma\left(\Lambda^{0} L^{\vee}\right) \otimes_{R} \mathscr{D}_{\text {poly }}^{0} \stackrel{\llbracket-\delta,-\rrbracket}{\longrightarrow} \Gamma\left(\Lambda^{1} L^{\vee}\right) \otimes_{R} \mathscr{D}_{\text {poly }}^{0} \stackrel{\llbracket-\delta,-\rrbracket}{\longrightarrow} \Gamma\left(\Lambda^{2} L^{\vee}\right) \otimes_{R} \mathscr{D}_{\text {poly }}^{0} \stackrel{\llbracket-\delta,-\rrbracket}{\longrightarrow} \cdots \\
& \llbracket m,-\rrbracket \uparrow \quad-\llbracket m,-\rrbracket \uparrow \quad \llbracket m,-\rrbracket \uparrow \\
& \Gamma\left(\Lambda^{0} L^{\vee}\right) \otimes_{R} \mathscr{D}_{\text {poly }}^{-1} \stackrel{\llbracket-\delta,-\rrbracket}{\longrightarrow} \Gamma\left(\Lambda^{1} L^{\vee}\right) \otimes_{R} \mathscr{D}_{\text {poly }}^{-1} \stackrel{\llbracket-\delta,-\rrbracket}{\longrightarrow} \Gamma\left(\Lambda^{2} L^{\vee}\right) \otimes_{R} \mathscr{D}_{\text {poly }}^{-1} \stackrel{\llbracket-\delta,-\rrbracket}{\longrightarrow} \cdots
\end{aligned}
$$

is a double complex. 
Its total complex

$$
\cdots \rightarrow \operatorname{tot}^{n}\left(\Gamma\left(\Lambda^{\bullet} L^{\vee}\right) \otimes_{R} \mathscr{D}_{\text {poly }}^{\bullet} \stackrel{\llbracket-\delta+m,-\rrbracket}{\longrightarrow} \operatorname{tot}^{n+1}\left(\Gamma\left(\Lambda^{\bullet} L^{\vee}\right) \otimes_{R} \mathscr{D}_{\text {poly }}^{\bullet}\right) \rightarrow \cdots\right.
$$

admits the descending filtration $\mathscr{F}_{0} \supset \mathscr{F}_{1} \supset \mathscr{F}_{2} \supset \mathscr{F}_{3} \supset \cdots$ defined by

$$
\mathscr{F}_{m}=\bigoplus_{k=0}^{\operatorname{rk}(L)} \Gamma\left(\Lambda^{k}\left(L^{\vee}\right)\right) \otimes_{R} \varphi\left(\bigoplus_{q=-1}^{\infty} \Gamma(\hat{S}^{\geqslant m-k}\left(B^{\vee}\right) \otimes \underbrace{S(B) \otimes \cdots \otimes S(B)}_{q+1 \text { factors }})\right) .
$$

Here $\varphi$ is as in (32).

We shall denote by $\tau_{\natural}, \sigma_{\natural}$ and $h_{\natural}$ the maps defined by the following commutative diagrams (where $\tau$, $\sigma$, and $h$ are the maps introduced in Section 2.2 and $\varphi$ is the identification (32)):

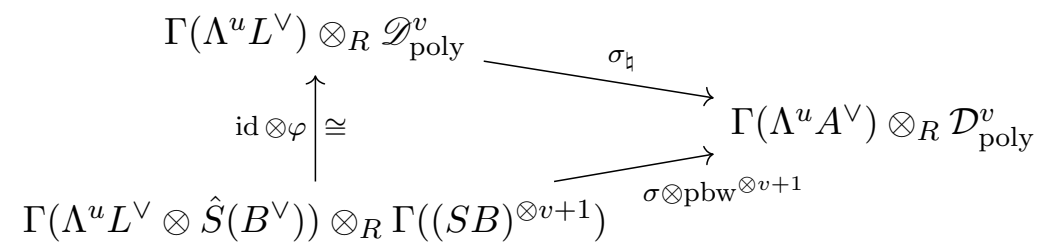

$$
\begin{aligned}
& \begin{array}{c}
\Gamma\left(\Lambda^{u} A^{\vee}\right) \otimes_{R} \mathcal{D}_{\text {poly }}^{v} \underset{\tau \otimes\left(\text { pbw }^{-1}\right)^{\otimes v+1} \underset{\tau_{\sharp}}{\Gamma}\left(\Lambda^{u} L^{\vee} \otimes \hat{S}\left(B^{\vee}\right)\right) \otimes_{R} \Gamma\left((S B)^{\otimes v+1}\right)}{\longrightarrow} \begin{array}{c}
\Gamma\left(\Lambda^{u} L^{\vee}\right) \otimes_{R} \mathscr{D}_{\text {poly }}^{v} \\
\text { id } \otimes \varphi \uparrow
\end{array}
\end{array} \\
& \Gamma\left(\Lambda^{u} L^{\vee}\right) \otimes_{R} \mathscr{D}_{\text {poly }}^{v} \longrightarrow h_{\natural} \longrightarrow \Gamma\left(\Lambda^{u-1} L^{\vee}\right) \otimes_{R} \mathscr{D}_{\text {poly }}^{v} \\
& \operatorname{id} \otimes \varphi \uparrow \cong \quad \operatorname{id} \otimes \varphi \uparrow \cong \\
& \Gamma\left(\Lambda^{u} L^{\vee} \otimes \hat{S}\left(B^{\vee}\right)\right) \otimes_{R} \Gamma\left((S B)^{\otimes v+1}\right) \underset{h \otimes \mathrm{id}}{\longrightarrow} \Gamma\left(\Lambda^{u-1} L^{\vee} \otimes \hat{S}\left(B^{\vee}\right)\right) \otimes_{R} \Gamma\left((S B)^{\otimes v+1}\right)
\end{aligned}
$$

The following proposition can be easily verified.

Proposition 3.14. The cochain complex ( tot $\left.\left(\Gamma\left(\Lambda^{\bullet} L^{\vee}\right) \otimes_{R} \mathscr{D}_{\text {poly }}^{\bullet}\right), \llbracket-\delta+m,-\rrbracket\right)$ contracts onto $\left(\right.$ tot $\left(\Gamma\left(\Lambda^{\bullet} A^{\vee}\right) \otimes_{R} \mathcal{D}_{\text {poly }}^{\bullet}\right)$, id $\left.\otimes d_{\mathscr{H}}\right)$. More precisely, we have a filtered contraction

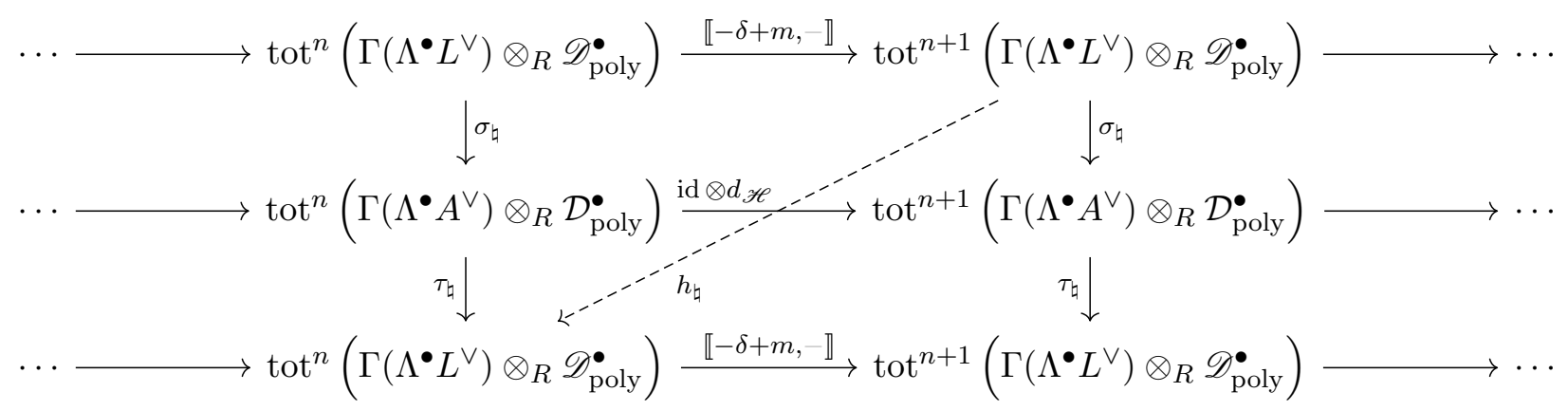

where $\sigma_{\natural}, \tau_{\natural}$ and $h_{\natural}$ are the maps defined by the commutative diagrams (38), (39), and (40).

Lemma 3.15. The contraction $\left(\tau_{\natural}, \sigma_{\natural}, h_{\natural}\right)$ in Proposition 3.14 is a semifull algebra contraction (where the associative product on both sides is the cup product). Moreover, the maps $\tau_{\natural}$ and $\sigma_{\natural}$ respect the cup products. 
Proof. This follows easily from the definitions and the corresponding statements for $(\tau, \sigma, h)$ - see Lemma 2.10.

Remark 3.16. For future reference, we point out that the same maps $\left(\tau_{\natural}, \sigma_{\natural}, h_{\natural}\right)$ also define a filtered contraction of $\left(\operatorname{tot}\left(\Gamma\left(\Lambda^{\bullet} L^{\vee}\right) \otimes_{R} \mathscr{D}_{\text {poly }}^{\bullet}\right), \llbracket-\delta,-\rrbracket\right)$ onto $\left(\operatorname{tot}\left(\Gamma\left(\Lambda^{\bullet} A^{\vee}\right) \otimes_{R} \mathcal{D}_{\text {poly }}^{\bullet}\right), 0\right)$. As for Proposition 3.14, we leave the verification of this claim as an easy exercise for the reader.

Lemma 3.17. The diagram

$$
\begin{gathered}
\Gamma\left(\Lambda^{p} L^{\vee}\right) \otimes_{R} \mathscr{D}_{\text {poly }}^{v+1} \stackrel{\llbracket \rho,-\rrbracket}{\longrightarrow} \Gamma\left(\Lambda^{p+1} L^{\vee}\right) \otimes_{R} \mathscr{D}_{\text {poly }}^{v+1} \\
(-1)^{p} \llbracket m,-\rrbracket \mid \\
\Gamma\left(\Lambda^{p} L^{\vee}\right) \otimes_{R} \mathscr{D}_{\text {poly }}^{v} \stackrel{\llbracket \rho,-\rrbracket}{\longrightarrow} \Gamma\left(\Lambda^{p+1} \llbracket m,-\rrbracket \mid\right.
\end{gathered}
$$

commutes.

Sketch of proof. We have $\llbracket \rho, m \rrbracket=0$ because, for every $l \in \Gamma(L)$, the operator $\iota_{l} \rho$ is a derivation for the multiplication $m$ on $C^{\infty}(L[1] \oplus B)$.

It follows from Proposition 3.13 and Lemma 3.17 that

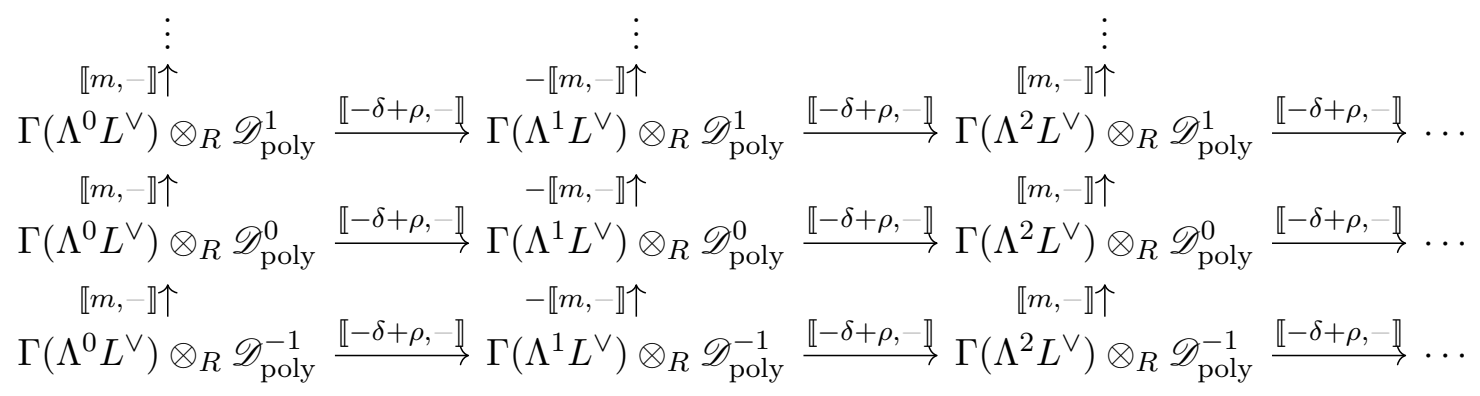

is a double complex.

Indeed, the operator $\llbracket \rho,-\rrbracket$ is a perturbation of the filtered complex

$$
\cdots \rightarrow \operatorname{tot}^{n}\left(\Gamma\left(\Lambda^{\bullet} L^{\vee}\right) \otimes_{R} \mathscr{D}_{\text {poly }}^{\bullet} \stackrel{\llbracket-\delta+m,-\rrbracket}{\longrightarrow} \operatorname{tot}^{n+1}\left(\Gamma\left(\Lambda^{\bullet} L^{\vee}\right) \otimes_{R} \mathscr{D}_{\text {poly }}^{\bullet}\right) \rightarrow \cdots\right.
$$

Proposition 3.18. There exists a contraction

$$
\left(\operatorname{tot}\left(\Gamma\left(\Lambda^{\bullet} A^{\vee}\right) \otimes_{R} \mathcal{D}_{\text {poly }}^{\bullet}\right), d_{A}^{\mathcal{U}}+\mathfrak{d}_{\mathscr{H}}\right) \underset{\sigma_{\natural}}{\stackrel{\breve{\tau}_{\natural}}{\rightleftarrows}}\left(\operatorname{tot}\left(\Gamma\left(\Lambda^{\bullet} L^{\vee}\right) \otimes_{R} \mathscr{D}_{\text {poly }}^{\bullet}\right), \llbracket Q+m,-\rrbracket\right) \supset \breve{h}_{\natural}
$$

— recall that $\mathfrak{d}_{\mathscr{H}}:=\mathrm{id} \otimes d_{\mathscr{H}}$. More precisely, we have the (filtered) contraction

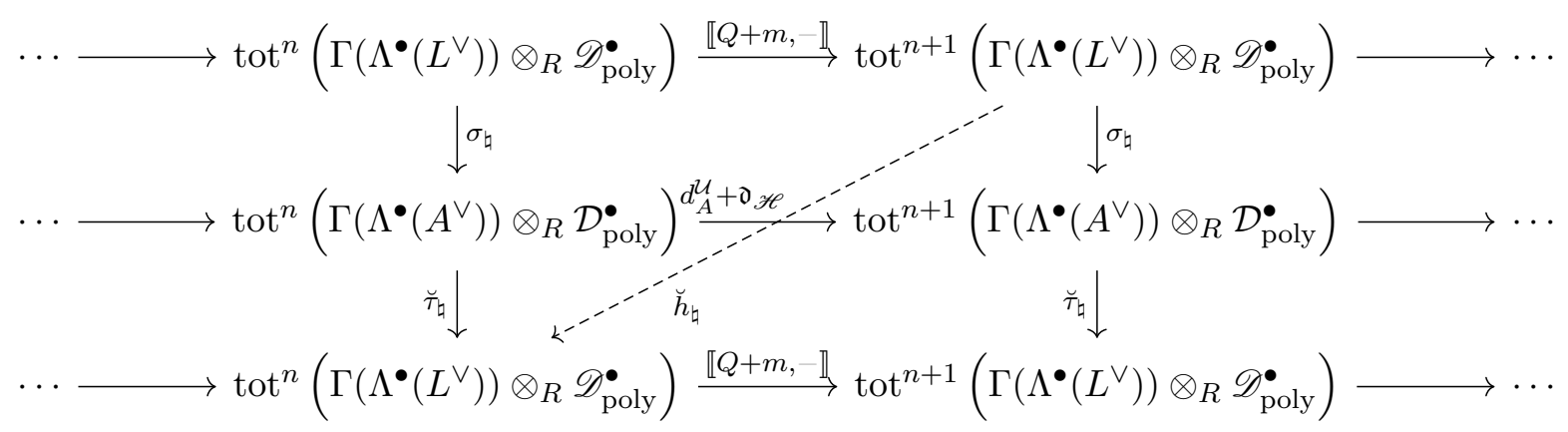

where $\breve{\tau}_{\natural}=\sum_{l=0}^{\infty}\left(h_{\natural} \circ \llbracket \rho,-\rrbracket\right)^{l} \circ \tau_{\natural}$ and $\breve{h}_{\natural}=\sum_{l=0}^{\infty}\left(h_{\natural} \circ \llbracket \rho,-\rrbracket\right)^{l} \circ h_{\natural}$.

Moreover, the cochain maps $\breve{\tau}_{\natural}$ and $\sigma_{\natural}$ respect the cup products on both sides. 
As an immediate consequence of Proposition 3.18, by considering the bigradings on both sides of (41), we obtain the following

Corollary 3.19. For every $k \geqslant-1$, we have a contraction

$$
\left(\Gamma\left(\Lambda^{\bullet} A^{\vee}\right) \otimes_{R} \mathcal{D}_{\text {poly }}^{k}, d_{A}^{\mathcal{U}}\right) \underset{\sigma_{\natural}}{\stackrel{\breve{\tau}_{\natural}}{\rightleftarrows}}\left(\Gamma\left(\Lambda^{\bullet} L^{\vee}\right) \otimes_{R} \mathscr{D}_{\text {poly }}^{k}, \llbracket Q,-\rrbracket\right) \breve{h}_{\natural}
$$

The case $k=-1$ was established in [45, Proposition 5.4].

The proof of Proposition 3.18 requires the following technical results.

Lemma 3.20. Let $\mathrm{pr}_{0}$ denote the canonical projection $\hat{S}\left(B^{\vee}\right) \otimes S(B) \rightarrow S^{0}\left(B^{\vee}\right) \otimes S(B)$. For all $a \in \Gamma(A)$ and $J \in \mathbb{N}_{0}^{r}$, we have

$$
\operatorname{pr}_{0}\left(\llbracket \nabla_{a}^{\xi}, \partial^{J} \rrbracket\right)=\nabla_{a}^{\xi}\left(\partial^{J}\right)
$$

Proof. We have seen that, for all $a \in \Gamma(A)$, the operator $\nabla_{a}^{\xi}$ is a derivation of $\Gamma\left(\hat{S}\left(B^{\vee}\right)\right)$, which stabilizes the filtration $\Gamma\left(\hat{S}^{\geqslant n}\left(B^{\vee}\right)\right)$. Therefore, there exist local sections $\theta_{k}^{M}$ of $L^{\vee}$ such that

$$
\nabla_{a}^{\xi} \chi_{k}=\sum_{\substack{M \in \mathbb{N}_{0}^{r} \\|M| \geqslant 1}} \iota_{a} \theta_{k}^{M} \cdot \chi^{M}
$$

It follows that $\nabla_{a}^{\xi}$ may be regarded as a section of $\hat{S}^{\geqslant 1}\left(B^{\vee}\right) \otimes B$ :

$$
\nabla_{a}^{\xi}=\sum_{k=1}^{r}\left(\sum_{\substack{M \in \mathbb{N}_{0}^{r} \\|M| \geqslant 1}} \iota_{a} \theta_{k}^{M} \cdot \chi^{M}\right) \partial_{k}
$$

On one hand, it follows from

$$
\llbracket \nabla_{a}^{\xi}, \partial^{J} \rrbracket=\nabla_{a}^{\xi} \star \partial^{J}-\partial^{J} \star \nabla_{a}^{\xi}=\sum_{k=1}^{r} \sum_{|M| \geqslant 1} \iota_{a} \theta_{k}^{M} \cdot \chi^{M} \partial^{J+e_{k}}-\sum_{k=1}^{r} \sum_{|M| \geqslant 1} \iota_{a} \theta_{k}^{M} \cdot\left(\partial^{J} \star \chi^{M} \partial_{k}\right),
$$

that

$$
\begin{aligned}
\operatorname{pr}_{0}\left(\llbracket \nabla_{a}^{\xi}, \partial^{J} \rrbracket\right) & =-\sum_{k=1}^{r} \sum_{|M| \geqslant 1} \iota_{a} \theta_{k}^{M} \frac{J !}{M !(J-M) !} \partial^{M}\left(\chi^{M}\right) \cdot \partial^{J-M+e_{k}} \\
& =-\sum_{k=1}^{r} \sum_{|M| \geqslant 1} \iota_{a} \theta_{k}^{M} \frac{J !}{(J-M) !} \cdot \partial^{J-M+e_{k}} .
\end{aligned}
$$

On the other hand, it follows from

$$
0=\varrho_{a} \underbrace{\left\langle\chi^{K} \mid \partial^{J}\right\rangle}_{K ! \cdot \delta_{K, J}}=\left\langle\nabla_{a}^{\xi} \chi^{K} \mid \partial^{J}\right\rangle+\left\langle\chi^{K} \mid \nabla_{a}^{\xi} \partial^{J}\right\rangle
$$


that

$$
\begin{aligned}
\nabla_{a}^{\xi}\left(\partial^{J}\right) & =\sum_{K} \frac{1}{K !}\left\langle\chi^{K} \mid \nabla_{a}^{\xi} \partial^{J}\right\rangle \partial^{K} \\
& =-\sum_{K} \frac{1}{K !}\left\langle\nabla_{a}^{\xi} \chi^{K} \mid \partial^{J}\right\rangle \partial^{K} \\
& =-\sum_{K} \frac{1}{K !}\left\langle\sum_{k} K_{k} \chi^{K-e_{k}} \nabla_{a}^{\xi} \chi_{k} \mid \partial^{J}\right\rangle \partial^{K} \\
& =-\sum_{K} \frac{1}{K !} \sum_{k} K_{k} \sum_{|M| \geqslant 1} \iota_{a} \theta_{k}^{M} \underbrace{\left\langle\chi^{K-e_{k}+M} \mid \partial^{J}\right\rangle}_{J ! \cdot \delta_{K-e_{k}+M, J}} \partial^{K} \\
& =-\sum_{k} \sum_{|M| \geqslant 1} \frac{J !}{\left(J-M+e_{k}\right) !}\left(J_{k}-M_{k}+1\right) \iota_{a} \theta_{k}^{M} \partial^{J-M+e_{k}} \\
& =-\sum_{k} \sum_{|M| \geqslant 1} \frac{J !}{(J-M) !} \iota_{a} \theta_{k}^{M} \partial^{J-M+e_{k}} .
\end{aligned}
$$

The proof is complete.

Lemma 3.21. $\sigma_{\natural} \circ \llbracket \rho,-\rrbracket \circ \tau_{\natural}=d_{A}^{\mathcal{U}}$

Proof. Let $\left(l_{k}\right)_{k \in\{1, \ldots, \mathrm{rk} L\}}$ denote any local frame for $L$ and let $\left(\lambda_{k}\right)_{k \in\{1, \ldots, \mathrm{rk} L\}}$ denote the dual local frame for $L^{\vee}$. Likewise let $\left(a_{k}\right)_{k \in\{1, \ldots, \text { rk } A\}}$ denote any local frame for $A$ and let $\left(\alpha_{k}\right)_{k \in\{1, \ldots, \text { rk } A\}}$ denote the dual local frame for $A^{\vee}$. For all $\omega \in \Gamma\left(\Lambda^{\bullet} A^{\vee}\right), n \in \mathbb{N}$, and $J_{0}, \ldots, J_{n} \in \mathbb{N}_{0}^{r}$, we have

$$
\begin{aligned}
& \sigma_{\natural}\left(\llbracket \rho, \tau_{\natural}\left(\omega \otimes \operatorname{pbw}\left(\partial^{J_{0}}\right) \otimes \cdots \otimes \operatorname{pbw}\left(\partial^{J_{n}}\right)\right) \rrbracket\right) \\
&=\sigma_{\natural}\left(\llbracket \rho, p^{\top} \omega \otimes \varphi\left(1 \otimes \partial^{J_{0}} \otimes \cdots \otimes \partial^{J_{n}}\right) \rrbracket\right) \\
&=\sigma_{\natural}\left(d_{L}\left(p^{\top} \omega\right) \otimes \varphi\left(1 \otimes \partial^{J_{0}} \otimes \cdots \otimes \partial^{J_{n}}\right)\right. \\
&\left.\quad+\sum_{k} \lambda_{k} \wedge p^{\top} \omega \otimes \llbracket \nabla_{l_{k}}-\iota_{l_{k}} \Xi, \varphi\left(1 \otimes \partial^{J_{0}} \otimes \cdots \otimes \partial^{J_{n}}\right) \rrbracket\right) \\
&=\sigma\left(d_{L}\left(p^{\top} \omega\right) \otimes 1\right) \otimes \operatorname{pbw}\left(\partial^{J_{0}}\right) \otimes \cdots \otimes \operatorname{pbw}\left(\partial^{J_{n}}\right) \\
& \quad+\sum_{k} \sigma_{\natural}\left(p^{\top} \alpha_{k} \wedge p^{\top} \omega \otimes \llbracket \nabla_{a_{k}}^{\xi}, \varphi\left(1 \otimes \partial^{J_{0}} \otimes \cdots \otimes \partial^{J_{n}}\right) \rrbracket\right) \\
&=d_{A} \omega \otimes \operatorname{pbw}\left(\partial^{J_{0}}\right) \otimes \cdots \otimes \operatorname{pbw}\left(\partial^{J_{n}}\right) \\
& \quad+\sum_{k} \sigma_{\natural}\left(p^{\top}\left(\alpha_{k} \wedge \omega\right) \otimes \varphi\left\{\sum_{t=0}^{n} 1 \otimes \partial^{J_{0}} \otimes \cdots \otimes \llbracket \nabla_{a_{k}}^{\xi}, \partial^{J_{t}} \rrbracket \otimes \cdots \otimes \partial^{J_{n}}\right\}\right) \\
&=d_{A} \omega \otimes \operatorname{pbw}\left(\partial^{J_{0}}\right) \otimes \cdots \otimes \operatorname{pbw}\left(\partial^{J_{n}}\right) \\
& \quad+\sum_{k} \sum_{t=0}^{n} \alpha_{k} \wedge \omega \otimes \operatorname{pbw}\left(\partial^{J_{0}}\right) \otimes \cdots \otimes \operatorname{pbw}\left(\operatorname{pr}_{0} \llbracket \nabla_{a_{k}}^{\xi}, \partial^{J_{t}} \rrbracket\right) \otimes \cdots \otimes \operatorname{pbw}\left(\partial^{J_{n}}\right) .
\end{aligned}
$$

It follows from Lemma 3.20 that

$$
\operatorname{pbw}\left(\operatorname{pr}_{0} \llbracket \nabla_{a_{k}}^{\xi}, \partial^{J_{t}} \rrbracket\right)=\operatorname{pbw}\left(\nabla_{a_{k}}^{\xi}\left(\partial^{J_{t}}\right)\right)=a_{k} \cdot \operatorname{pbw}\left(\partial^{J_{t}}\right) .
$$

Hence, we conclude that $\sigma_{\natural} \circ \llbracket \rho,-\rrbracket \circ \tau_{\natural}=d_{A}^{\mathcal{U}}$. 
Proof of Proposition 3.18. We proceed by homological perturbation — see Lemma A.1 and also [45, Lemma A.1]. Starting from the filtered contraction of Proposition 3.14, it suffices to perturb the coboundary operator $\llbracket-\delta+m,-\rrbracket$ by the operator $\llbracket \rho,-\rrbracket$. One checks that $\sigma_{\natural} \circ \llbracket \rho,-\rrbracket \circ h_{\natural}=0$. Therefore, we obtain

$$
\breve{\sigma}_{\natural}:=\sum_{l=0}^{\infty} \sigma_{\natural} \circ\left(\llbracket \rho,-\rrbracket \circ h_{\natural}\right)^{l}=\sigma_{\natural}
$$

and, making use of Lemma 3.21,

$$
\vartheta:=\sum_{l=0}^{\infty} \sigma_{\natural} \circ\left(\llbracket \rho,-\rrbracket \circ h_{\natural}\right)^{l} \circ \llbracket \rho,-\rrbracket \circ \tau_{\natural}=\sigma_{\natural} \circ \llbracket \rho,-\rrbracket \circ \tau_{\natural}=d_{A}^{\mathcal{U}} .
$$

The result follows immediately since $-\delta+\rho=Q$.

As in the proof of Proposition 3.4, applying Lemmas 3.15 and A.3, we conclude that $\left(\breve{\tau}_{\natural}, \sigma_{\natural}, \breve{h}_{\natural}\right)$ is a semifull algebra contraction. Since the differential $\llbracket Q+m,-\rrbracket=\llbracket Q,-\rrbracket+d_{\mathscr{H}}$ is a derivation with respect to the cup product ${ }^{4}$, according to Lemma A.4, we conclude that $\breve{\tau}_{\natural}$ is an algebra morphism. The fact that $\sigma_{\natural}$ is an algebra morphism is already contained in Lemma 3.15.

The next proposition gives an alternative characterization of the map $\breve{\tau}_{\natural}$ as the solution of an initial value problem.

Proposition 3.22. Given $x \in \Gamma\left(\Lambda^{\bullet} A^{\vee}\right) \otimes_{R} \mathcal{D}_{\text {poly }}^{\diamond}$ and $y \in \Gamma\left(\Lambda^{\bullet} L^{\vee}\right) \otimes_{R} \mathscr{D}_{\text {poly }}^{\diamond}$, we have

$$
\breve{\tau}_{\natural}(x)=y \quad \text { if and only if } \quad\left\{\begin{array} { l } 
{ h _ { \natural } ( y ) = 0 } \\
{ h _ { \natural } ( \llbracket Q , y \rrbracket ) = 0 } \\
{ \sigma _ { \natural } ( y ) = x }
\end{array} \quad \text { if and only if } \quad \left\{\begin{array}{l}
\llbracket \eta, y \rrbracket=0 \\
\llbracket[\eta, Q], y \rrbracket=0 \\
\sigma_{\natural}(y)=x
\end{array}\right.\right.
$$

The derivation $\eta$ was defined in Equation (25). The proof of Proposition 3.22 is similar to the proof of Proposition 3.8 and is therefore omitted.

Proposition 3.23. The restriction of the map $\breve{\tau}_{\natural}$ of Corollary3.19 to differential (rather than polydifferential) operators is a morphism of coalgebras

$$
\breve{\tau}_{\natural}: \Gamma\left(\Lambda^{\bullet} A^{\vee}\right) \otimes_{R} \mathcal{D}_{\text {poly }}^{0} \rightarrow \Gamma\left(\Lambda^{\bullet} L^{\vee}\right) \otimes_{R} \mathscr{D}_{\text {poly }}^{0} .
$$

Proof. Since, according to Proposition (3.18), $\breve{\tau}_{\natural}$ respects the cup products, we have the commutative diagram

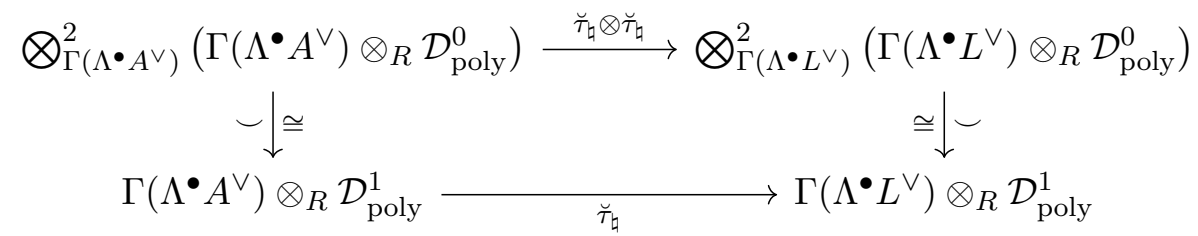

in which, owing to the very definition of the cup products, the two vertical arrows are isomorphisms. Denoting by $\breve{\Delta}$ the composition of the comultiplication and the cup product, we are thus led to show that the diagram

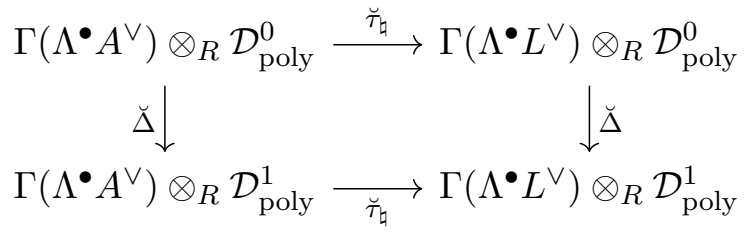

\footnotetext{
${ }^{4}$ Although the Gerstenhaber bracket $\llbracket-,-\rrbracket$ is not a biderivation with respect to the cup product, but only a biderivation up to homotopy, it is known that $\llbracket m,-\rrbracket=d_{\mathscr{H}}$ is indeed a derivation with respect to the cup product - see [19, Equation (20)].
} 
commutes.

By virtue of Proposition 3.22, it suffices to show that the three identities

$$
\llbracket \eta, \breve{\Delta} \circ \breve{\tau}_{\natural}(x) \rrbracket=0, \quad \llbracket[\eta, Q], \breve{\Delta} \circ \breve{\tau}_{\natural}(x) \rrbracket=0, \quad \text { and } \quad \sigma_{\natural}\left(\breve{\Delta} \circ \breve{\tau}_{\natural}(x)\right)=\breve{\Delta}(x)
$$

hold for every $x \in \Gamma\left(\Lambda^{\bullet} A^{\vee}\right) \otimes_{R} \mathcal{D}_{\text {poly }}^{0}$.

Consider the dg Hopf algebroid $\mathcal{U}(\mathcal{F})$ arising from the Fedosov dg Lie algebroid $\mathcal{F} \rightarrow \mathcal{M}$. Given any $b \in \Gamma(\mathcal{F})=\Gamma\left(\Lambda^{\bullet} L^{\vee} \otimes \hat{S}\left(B^{\vee}\right) \otimes B\right)$ and $u \in \mathcal{U}(\mathcal{F})=\Gamma\left(\Lambda^{\bullet} L^{\vee} \otimes \hat{S}\left(B^{\vee}\right) \otimes S B\right)$, we have

$$
\begin{aligned}
& \llbracket b, \breve{\Delta}(u) \rrbracket=b \star\left(\sum_{(u)} u_{(1)} \smile\right.\left.u_{(2)}\right)-\left(\sum_{(u)} u_{(1)} \smile u_{(2)}\right) \star b \\
&= \sum_{(u)}\left(\left(b \circ u_{(1)}\right) \smile u_{(2)}+u_{(1)} \smile\left(b \circ u_{(2)}\right)\right) \\
&-\sum_{(u)}\left(\left(u_{(1)} \circ b\right) \smile u_{(2)}+u_{(1)} \smile\left(u_{(2)} \circ b\right)\right) \\
&=\breve{\Delta}(b \circ u)-\breve{\Delta}(u \circ b)=\breve{\Delta}(\llbracket b, u \rrbracket) .
\end{aligned}
$$

This fact together with Proposition 3.22 immediately implies that

$$
\llbracket \eta, \breve{\Delta}\left(\breve{\tau}_{\natural}(x)\right) \rrbracket=\breve{\Delta}\left(\llbracket \eta, \breve{\tau}_{\natural}(x) \rrbracket\right)=0
$$

and

$$
\llbracket[\eta, Q], \breve{\Delta}\left(\breve{\tau}_{\natural}(x)\right) \rrbracket=\breve{\Delta}\left(\llbracket[\eta, Q], \breve{\tau}_{\natural}(x) \rrbracket\right)=0 .
$$

Furthermore, since $\sigma_{\natural}$ is a morphism of coalgebras and $\sigma_{\natural} \circ \breve{\tau}_{\natural}=\mathrm{id}$, we have

$$
\sigma_{\natural} \circ \breve{\Delta} \circ \breve{\tau}_{\natural}(x)=\breve{\Delta} \circ \sigma_{\natural} \circ \breve{\tau}_{\natural}(x)=\breve{\Delta}(x) .
$$

The proof is complete.

Finally, we have the following

Proposition 3.24. Given a Lie pair $(L, A)$, each choice of a splitting $j: B \rightarrow L$ of the short exact sequence of vector bundles $0 \rightarrow A \rightarrow L \rightarrow B \rightarrow 0$ and of a torsion-free $L$-connection $\nabla$ on $B$ determines

(1) an $L_{\infty}$ algebra structure on tot $\left(\Gamma\left(\Lambda^{\bullet} A^{\vee}\right) \otimes_{R} \mathcal{D}_{\text {poly }}^{\bullet}\right)$ with the operator $d_{A}^{\mathcal{U}}+\mathfrak{d}_{\mathscr{H}}$ as unary bracket; (2) and a Gerstenhaber algebra structure on $\mathbb{H}_{\mathrm{CE}}^{\bullet}\left(A, \mathcal{D}_{\text {poly }}^{\bullet}\right)$, the cohomology of the total complex

$$
\left(\operatorname{tot}\left(\Gamma\left(\Lambda^{\bullet} A^{\vee}\right) \otimes_{R} \mathcal{D}_{\text {poly }}^{\bullet}\right), d_{A}^{\mathcal{U}}+\mathfrak{d}_{\mathscr{H}}\right),
$$

where the Lie bracket is induced by the binary bracket of the $L_{\infty}$ algebra structure on tot $\left(\Gamma\left(\Lambda^{\bullet} A^{\vee}\right) \otimes_{R} \mathcal{D}_{\text {poly }}^{\bullet}\right)$ and the multiplication by the cup product (8).

Proof. Applying the homotopy transfer theorem for $L_{\infty}$ algebras $[5,22,17]$ to the $L_{\infty}$ algebra obtained in Proposition 2.16 and the contraction obtained in Proposition 3.18, we get an induced $L_{\infty}$ algebra structure on tot $\left(\Gamma\left(\Lambda^{\bullet} A^{\vee}\right) \otimes_{R} \mathcal{D}_{\text {poly }}^{\bullet}\right)$, where the unary bracket is the differential $d_{A}^{\mathcal{U}}+\mathfrak{d}_{\mathscr{H}}$. This proves (1).

For (2), we notice that at the level of cohomology $\breve{\tau}_{\natural}$ and $\sigma_{\natural}$ induce isomorphisms of graded spaces which are compatible with both the induced graded Lie algebra structures (by construction, since these are related via homotopy transfer along $\left.\left(\breve{\tau}_{\natural}, \sigma_{\natural}, \breve{h}_{\natural}\right)\right)$ and the induced graded associative algebra structures (by Proposition 3.18). It follows at once that the induced graded Lie algebra and graded associative algebra structures make $\mathbb{H}_{\mathrm{CE}}^{\bullet}\left(A, \mathcal{D}_{\text {poly }}^{\bullet}\right)$ into a Gerstenhaber algebra, since the same is true for $\mathbb{H}^{\bullet}\left(\operatorname{tot}\left(\Gamma\left(\Lambda^{\bullet} L^{\vee}\right) \otimes_{R} \mathscr{D}_{\text {poly }}^{\bullet}\right), \llbracket Q+m,-\rrbracket\right)$, according to Proposition 2.16. In particular, this shows that the cup product on $\mathbb{H}_{\mathrm{CE}}^{\bullet}\left(A, \mathcal{D}_{\text {poly }}^{\bullet}\right)$ is graded commutative, cf. the discussion in Remark 1.4. 
3.3. Uniqueness of the $L_{\infty}$ structure. A priori, the Gerstenhaber algebra structures on $\mathbb{H}_{\mathrm{CE}}^{\bullet}\left(A, \mathcal{T}_{\text {poly }}^{\bullet}\right)$ and $\mathbb{H}_{\mathrm{CE}}^{\bullet}\left(A, \mathcal{D}_{\text {poly }}^{\bullet}\right)$ in Propositions 3.9 and 3.24 are not canonical, as their constructions depend on a choice of a splitting $j: B \rightarrow L$ of the short exact sequence $0 \rightarrow A \rightarrow L \rightarrow B \rightarrow 0$ and a torsion-free $L$-connection $\nabla$ on $B$. The aim of this section is to complete the proof of Theorem A from the introduction and show that both Gerstenhaber algebras are indeed canonical.

As observed at the end of Section 2.2, the Fedosov dg manifolds arising from different choices of a splitting and a connection are isomorphic with each other (and we made the isomorphism explicit in terms of the associated PBW maps). There are induced isomorphisms between the Fedosov dg Lie algebroids, hence between the corresponding algebras of polyvector fields and polydifferential operators from Propositions 2.15 and 2.16. We can make these isomorphisms explicit, once again in terms of the associated PBW maps. Throughout the present section we shall concentrate on the (harder) case of polydifferential operators - the proof for the case of polyvector fields is similar (see also [2]).

We consider two different choices $j_{1}, \nabla_{1}$ and $j_{2}, \nabla_{2}$ of a splitting of the short sequence $0 \rightarrow A \rightarrow L \rightarrow B \rightarrow 0$ and a torsion-free $L$-connection on $B$, together with the induced homological vector fields $Q_{1}$ and $Q_{2}$ on $\mathcal{M}=L[1] \oplus B$, as in Theorem 2.11, and the induced PBW isomorphisms $\mathrm{pbw}_{1}$ and $\mathrm{pbw}_{2}: \Gamma(S B) \rightarrow \frac{\mathcal{U}(L)}{\mathcal{U}(L) \Gamma(A)}$, as in the discussion preceding Theorem 2.13.

Recall the isomorphism of $R$-coalgebras

$$
\psi:=\mathrm{pbw}_{1}^{-1} \circ \mathrm{pbw}_{2}: \Gamma(S B) \rightarrow \Gamma(S B)
$$

and the dual isomorphism of $R$-algebras $\psi^{\vee}: \Gamma\left(\hat{S}\left(B^{\vee}\right)\right) \rightarrow \Gamma\left(\hat{S}\left(B^{\vee}\right)\right)$ introduced at the end of Section 2.2. There is an induced isomorphism $\psi_{*}: \mathscr{D}_{\text {poly }}^{\bullet} \rightarrow \mathscr{D}_{\text {poly }}^{\bullet}$ between the spaces of polydifferential operators, sending a polydifferential operator $D \in \mathscr{D}_{\text {poly }}^{k}$ to the one $\psi_{*}(D) \in \mathscr{D}_{\text {poly }}^{k}$ defined by

$$
\psi_{*}(D)\left(\chi^{I_{0}}, \ldots, \chi^{I_{k}}\right)=\psi^{\vee}\left(D\left(\left(\psi^{\vee}\right)^{-1}\left(\chi^{I_{0}}\right), \ldots,\left(\psi^{\vee}\right)^{-1}\left(\chi^{I_{k}}\right)\right)\right)
$$

for all $\chi^{I_{0}}, \ldots \chi^{I_{k}} \in \Gamma\left(\hat{S}\left(B^{\vee}\right)\right)$. By construction, $\psi_{*}$ is compatible with the Gerstenhaber bracket of polydifferential operators, and in fact

$$
\begin{aligned}
& \operatorname{id} \otimes \psi_{*}:\left(\operatorname{tot}\left(\Gamma\left(\Lambda^{\bullet} L^{\vee}\right) \otimes_{R} \mathscr{D}_{\text {poly }}^{\bullet}\right), \llbracket Q_{1}+m,-\rrbracket, \llbracket-,-\rrbracket\right) \\
& \rightarrow\left(\operatorname{tot}\left(\Gamma\left(\Lambda^{\bullet} L^{\vee}\right) \otimes_{R} \mathscr{D}_{\text {poly }}^{\bullet}\right), \llbracket Q_{2}+m,-\rrbracket, \llbracket-,-\rrbracket\right)
\end{aligned}
$$

is an isomorphism of dglas.

We shall need the following lemma.

Lemma 3.25. Under the identification $\varphi$ from (32), the isomorphism $\psi_{*}$ satisfies

$$
\varphi^{-1} \circ \psi_{*} \circ \varphi\left(\chi^{I} \otimes \partial_{J_{0}} \otimes \cdots \otimes \partial_{J_{k}}\right)=\chi^{I} \otimes \psi^{-1}\left(\partial_{J_{0}}\right) \otimes \cdots \otimes \psi^{-1}\left(\partial_{J_{k}}\right)+\xi,
$$

where $\xi \in \hat{S}^{>|I|}\left(B^{\vee}\right) \otimes S(B)^{\otimes k+1}$.

Proof. In terms of any pair of dual local frames $\left\{\chi^{I}\right\}_{I \in \mathbb{N}^{r}}$ and $\left\{\partial_{J}\right\}_{J \in \mathbb{N}^{r}}$ for $\hat{S}\left(B^{\vee}\right)$ and $S(B)$ respectively, the isomorphisms $\psi^{-1}: \Gamma(S B) \rightarrow \Gamma(S B)$ and $\left(\psi^{\vee}\right)^{-1}: \Gamma\left(\hat{S}\left(B^{\vee}\right)\right) \rightarrow \Gamma\left(\hat{S}\left(B^{\vee}\right)\right)$ are given by

$$
\psi^{-1}\left(\partial_{J}\right)=\sum_{K \in \mathbb{N}^{r}} \frac{1}{K !} \psi_{J}^{K} \partial_{K} \quad \text { and } \quad\left(\psi^{\vee}\right)^{-1}\left(\chi^{I}\right)=\sum_{K \in \mathbb{N}^{r}} \frac{1}{K !} \psi_{K}^{I} \chi^{K},
$$

where the $\psi_{J}^{I}$ are smooth functions on the base manifold (more precisely, on the open subset $U \subset M$ on which the local frames are defined).

Let $D$ and $D^{\prime} \in \mathscr{D}_{\text {poly }}^{k}$ be the polydifferential operators defined by

$$
D:=\psi_{*} \circ \varphi\left(\chi^{I} \otimes \partial_{J_{0}} \otimes \cdots \otimes \partial_{J_{k}}\right) \quad \text { and } \quad D^{\prime}:=\varphi\left(\chi^{I} \otimes \psi^{-1}\left(\partial_{J_{0}}\right) \otimes \cdots \otimes \psi^{-1}\left(\partial_{J_{k}}\right)\right) .
$$

We have to show that their difference $D-D^{\prime}$ sends $\Gamma\left(\hat{S}\left(B^{\vee}\right)\right)^{\otimes k+1}$ into $\Gamma\left(\hat{S}^{>|I|}\left(B^{\vee}\right)\right)$. 
For all $I, J, K \in \mathbb{N}^{r}$, we have

$$
\psi^{\vee}\left(\chi^{I}\right)=\chi^{I}+\text { terms in } \Gamma\left(S^{>|I|}\left(B^{\vee}\right)\right)
$$

and

$$
\partial_{J}\left(\chi^{K}\right)= \begin{cases}(K-J) ! \cdot \chi^{K-J} & \text { if } J \prec K \\ 0 & \text { otherwise }\end{cases}
$$

The partial order $\prec$ on $\mathbb{N}^{r}$ is defined as follows: $\left(j_{1}, j_{2}, \cdots, j_{r}\right) \prec\left(k_{1}, k_{2}, \cdots, k_{r}\right)$ if and only if $j_{p} \leqslant k_{p}$ for each $p \in\{1,2, \cdots, r\}$.

It follows that, for all $\chi^{I_{0}}, \ldots, \chi^{I_{k}} \in \Gamma\left(\hat{S}\left(B^{\vee}\right)\right)$,

$$
\begin{aligned}
D\left(\chi^{I_{0}}, \ldots, \chi^{I_{K}}\right) & =\psi^{\vee}\left(\chi^{I} \cdot \partial_{J_{0}}\left(\left(\psi^{\vee}\right)^{-1}\left(\chi^{I_{0}}\right)\right) \cdots \partial_{J_{K}}\left(\left(\psi^{\vee}\right)^{-1}\left(\chi^{I_{K}}\right)\right)\right) \\
& =\psi^{\vee}\left(\sum_{K_{0}, \ldots, K_{k} \in \mathbb{N}^{r}} \frac{1}{K_{0} ! \cdots K_{k} !} \psi_{K_{0}}^{I_{0}} \cdots \psi_{K_{k}}^{I_{k}} \chi^{I} \cdot \partial_{J_{0}}\left(\chi^{K_{0}}\right) \cdots \partial_{J_{k}}\left(\chi^{K_{k}}\right)\right) \\
& =\psi_{J_{0}}^{I_{0}} \cdots \psi_{J_{k}}^{I_{k}} \chi^{I}+\text { terms in } \Gamma\left(S^{>|I|}\left(B^{\vee}\right)\right),
\end{aligned}
$$

while

$$
\begin{aligned}
D^{\prime}\left(\chi^{I_{0}}, \ldots, \chi^{I_{K}}\right) & =\chi^{I} \cdot \psi^{-1}\left(\partial_{J_{0}}\right)\left(\chi^{I_{0}}\right) \cdots \psi^{-1}\left(\partial_{J_{K}}\right)\left(\chi^{I_{K}}\right) \\
& =\sum_{K_{0}, \ldots, K_{k} \in \mathbb{N}^{r}} \frac{1}{K_{0} ! \cdots K_{k} !} \psi_{J_{0}}^{K_{0}} \cdots \psi_{J_{k}}^{K_{k}} \chi^{I} \cdot \partial_{K_{0}}\left(\chi^{I_{0}}\right) \cdots \partial_{K_{k}}\left(\chi^{I_{k}}\right) \\
& =\psi_{J_{0}}^{I_{0}} \cdots \psi_{J_{k}}^{I_{k}} \chi^{I}+\text { terms in } \Gamma\left(S^{>|I|}\left(B^{\vee}\right)\right) .
\end{aligned}
$$

This concludes the proof of the lemma.

With these preparations, we are finally ready to complete the proof of Theorem A from the introduction. In light of Propositions 3.9 and 3.24, the only thing which remains to be shown is the following

Proposition 3.26. The $L_{\infty}$ algebra structures on tot $\left(\Gamma\left(\Lambda^{\bullet} A^{\vee}\right) \otimes_{R} \mathcal{T}_{\text {poly }}^{\bullet}\right)$ and tot $\left(\Gamma\left(\Lambda^{\bullet} A^{\vee}\right) \otimes_{R} \mathcal{D}_{\text {poly }}^{\bullet}\right)$ from Propositions 3.9 and 3.24, respectively, are independent of the involved choices up to an $L_{\infty}$ isomorphism with linear part the identity map. In particular, the induced Gerstenhaber algebra structures on $\mathbb{H}_{\mathrm{CE}}^{\bullet}\left(A, \mathcal{T}_{\text {poly }}^{\bullet}\right)$ and $\mathbb{H}_{\mathrm{CE}}^{\bullet}\left(A, \mathcal{D}_{\text {poly }}^{\bullet}\right)$ are independent of the involved choices.

Proof. We shall prove the proposition in detail for the $L_{\infty}$ algebra structure on tot $\left(\Gamma\left(\Lambda^{\bullet} A^{\vee}\right) \otimes_{R} \mathcal{D}_{\text {poly }}^{\bullet}\right)$. The claim for tot $\left(\Gamma\left(\Lambda^{\bullet} A^{\vee}\right) \otimes_{R} \mathcal{T}_{\text {poly }}^{\bullet}\right)$ can be proved by a similar reasoning, or by comparison with the results from [2], where in fact a stronger result is proven: the $L_{\infty}$ algebra structure on tot $\left(\Gamma\left(\Lambda^{\bullet} A^{\vee}\right) \otimes_{R} \mathcal{T}_{\text {poly }}^{\bullet}\right)$ is independent of the choice of $\nabla$ altogether, and is independent of the choice of $j$ up to an $L_{\infty}$ isomorphism with linear part the identity map (cf. [2, Propositions 4.9 and 4.17]).

Let $j_{1}, \nabla_{1}$ and $j_{2}, \nabla_{2}$ be two choices of a splitting and a connection. Each choice $j_{i}, \nabla_{i}$ (with $i \in\{1,2\})$ determines a homological vector field $Q_{i}$ on $L[1] \oplus B$, a Poincaré-Birkhoff-Witt isomorphism $\operatorname{pbw}_{i}: \Gamma(S B) \rightarrow \frac{\mathcal{U}(L)}{\mathcal{U}(L) \Gamma(A)}$, and a Dolgushev-Fedosov contraction

$$
\left(\operatorname{tot}\left(\Gamma\left(\Lambda^{\bullet} A^{\vee}\right) \otimes_{R} \mathcal{D}_{\text {poly }}^{\bullet}\right), d_{A}^{\mathcal{U}}+\mathfrak{d}_{\mathscr{H}}\right) \underset{\sigma_{\natural, i}}{\stackrel{\breve{\tau}_{\sharp, i}}{\rightleftarrows}}\left(\operatorname{tot}\left(\Gamma\left(\Lambda^{\bullet} L^{\vee}\right) \otimes_{R} \mathscr{D}_{\text {poly }}^{\bullet}\right), \llbracket Q_{i}+m,-\rrbracket\right) \bigvee \breve{h}_{\natural, i}
$$

as in Proposition 3.18. 
Together with an $L_{\infty}$ algebra structure $\Upsilon_{i}$ on $\left(\operatorname{tot}\left(\Gamma\left(\Lambda^{\bullet} A^{\vee}\right) \otimes_{R} \mathcal{D}_{\text {poly }}^{\bullet}\right), d_{A}^{\mathcal{U}}+\mathfrak{d} \mathscr{H}\right)$, homotopy transfer along the Dolgushev-Fedosov contraction (44) induces a pair of $L_{\infty}$ quasi-isomorphisms

$$
\begin{aligned}
& \operatorname{tot}\left(\Gamma\left(\Lambda^{\bullet} A^{\vee}\right) \otimes_{R} \mathcal{D}_{\text {poly }}^{\bullet}\right) \rightsquigarrow \operatorname{tot}\left(\Gamma\left(\Lambda^{\bullet} L^{\vee}\right) \otimes_{R} \mathscr{D}_{\text {poly }}^{\bullet}\right) \\
& \operatorname{tot}\left(\Gamma\left(\Lambda^{\bullet} L^{\vee}\right) \otimes_{R} \mathscr{D}_{\text {poly }}^{\bullet}\right) \rightsquigarrow \operatorname{tot}\left(\Gamma\left(\Lambda^{\bullet} A^{\vee}\right) \otimes_{R} \mathcal{D}_{\text {poly }}^{\bullet}\right)
\end{aligned}
$$

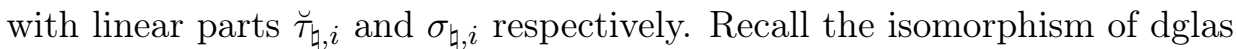

$$
\begin{aligned}
\left(\operatorname{tot}\left(\Gamma\left(\Lambda^{\bullet} L^{\vee}\right) \otimes_{R} \mathscr{D}_{\text {poly }}^{\bullet}\right), \llbracket Q_{1}+m,-\rrbracket, \llbracket-,-\rrbracket\right) \stackrel{\operatorname{id} \otimes \psi_{*}}{\longrightarrow} & \left(\operatorname{tot}\left(\Gamma\left(\Lambda^{\bullet} L^{\vee}\right) \otimes_{R} \mathscr{D}_{\text {poly }}^{\bullet}\right), \llbracket Q_{2}+m,-\rrbracket, \llbracket-,-\rrbracket\right)
\end{aligned}
$$

defined in (43). The two induced $L_{\infty}$ algebra structures on tot $\left(\Gamma\left(\Lambda^{\bullet} A^{\vee}\right) \otimes_{R} \mathcal{D}_{\text {poly }}^{\bullet}\right)$ are related by an $L_{\infty}$ morphism

$$
F:\left(\operatorname{tot}\left(\Gamma\left(\Lambda^{\bullet} A^{\vee}\right) \otimes_{R} \mathcal{D}_{\text {poly }}^{\bullet}\right), \Upsilon_{1}\right) \rightsquigarrow\left(\operatorname{tot}\left(\Gamma\left(\Lambda^{\bullet} A^{\vee}\right) \otimes_{R} \mathcal{D}_{\text {poly }}^{\bullet}\right), \Upsilon_{2}\right)
$$

with linear part $f_{1}=\sigma_{\natural, 2} \circ\left(\mathrm{id} \otimes \psi_{*}\right) \circ \breve{\tau}_{\natural, 1}$.

In order to conclude the proof, we only need to show that $f_{1}$ is the identity map. In fact, since an $L_{\infty}$ morphism $F$ is an isomorphism of $L_{\infty}$ algebras if and only if its linear part $f_{1}$ is an isomorphism between the underlying tangent complexes, this will show that $F$ is an $L_{\infty}$ isomorphism. Moreover, once we have proven that $f_{1}$ is the identity map, the second claim will also follow. In fact, denoting by $\llbracket-,-\rrbracket_{i}$ the Lie bracket on $\mathbb{H}_{\mathrm{CE}}^{\bullet}\left(A, \mathcal{D}_{\text {poly }}^{\bullet}\right)$ induced by the quadratic bracket of $\Upsilon_{i}, i=1,2$, in order to conclude the proof we only need to show $\llbracket-,-\rrbracket_{1}=\llbracket-,-\rrbracket_{2}$, since the associative product (the cup product) is already independent of the choices at the cochain level: but, $F$ being an $L_{\infty}$ morphism, its linear part $f_{1}$ commutes with the quadratic brackets of $\Upsilon_{1}, \Upsilon_{2}$, up to the homotopy $f_{2}$, thus the induced map on cohomology intertwines the two brackets $\llbracket-,-\rrbracket_{1}$ and $\llbracket-,-\rrbracket_{2}$.

Recall the identification $\varphi$ from (32), the map $\sigma: \Lambda^{\bullet} L^{\vee} \otimes \hat{S}\left(B^{\vee}\right) \rightarrow \Lambda^{\bullet} A^{\vee}$ from (24) and the commutative diagram

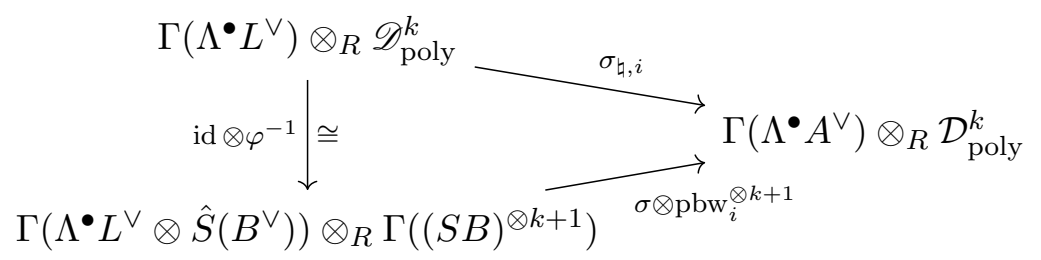

defining $\sigma_{\natural, i}$. According to Lemma 3.25, the maps $\varphi^{-1} \circ \psi_{*}$ and $\left(\operatorname{id} \otimes\left(\psi^{-1}\right)^{\otimes k+1}\right) \circ \varphi^{-1}$ from $\mathscr{D}_{\text {poly }}^{k}$ to $\Gamma\left(\hat{S}\left(B^{\vee}\right) \otimes S(B)^{\otimes k+1}\right)$ coincide up to terms in $\Gamma\left(\hat{S}^{\geqslant 1}\left(B^{\vee}\right) \otimes S(B)^{\otimes k+1}\right)$. Therefore, we obtain

$$
\sigma_{\natural, 2} \circ\left(\mathrm{id} \otimes \psi_{*}\right)=\left(\sigma \otimes \mathrm{pbw}_{2}^{\otimes k+1}\right) \circ\left(\mathrm{id} \otimes\left(\varphi^{-1} \circ \psi_{*}\right)\right)=\left(\sigma \otimes\left(\mathrm{pbw}_{2} \circ \psi^{-1}\right)^{\otimes k+1}\right) \circ\left(\mathrm{id} \otimes \varphi^{-1}\right)=\sigma_{\natural, 1}
$$

since $\Gamma\left(\Lambda^{\bullet} L^{\vee} \otimes \hat{S}^{\geqslant 1}\left(B^{\vee}\right)\right) \subset \operatorname{ker}(\sigma)$ and $\psi=\mathrm{pbw}_{1}^{-1} \circ \mathrm{pbw}_{2}$. It follows that

$$
f_{1}=\sigma_{\natural, 2} \circ\left(\mathrm{id} \otimes \psi_{*}\right) \circ \breve{\tau}_{\natural, 1}=\sigma_{\natural, 1} \circ \breve{\tau}_{\natural, 1}=\mathrm{id} \text {. }
$$

\section{MATCHED PAIR CASE}

This section is devoted to the proof of Theorem B, which was stated in the introduction. See Theorems 4.9 and 4.12 below.

Let $(L, A)$ be a Lie pair with quotient $B:=L / A$. Recall that, if a splitting $j: B \rightarrow L$ of the short exact sequence $0 \rightarrow A \stackrel{i}{\rightarrow} L \rightarrow B \rightarrow 0$ is given, whose image $j(B)$ happens to be a Lie subalgebroid of $L$, then $A$ and $B$ are said to form a matched pair of Lie algebroids - see [39,35] for more details. In such a situation, we write $L=A \bowtie B$ to stress that $A$ and $B$ - more precisely $i(A)$ and $j(B)$ - play symmetric roles as a pair of complementary Lie subalgebroids of the Lie algebroid $L$. In 
the case of matched pairs, the algebraic structures on the space of polyvector fields and the space of polydifferential operators described in Section 3 reduce to the natural ones described in Theorem B.

4.1. Dg Lie algebroid arising from a matched pair. Let $L=A \bowtie B$ be a matched pair of Lie algebroids over a manifold $M$. Consider the double vector bundle

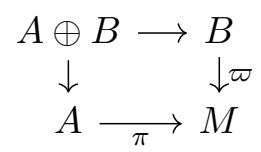

where the vector bundle $A \oplus B \rightarrow A$ is the pullback of the vector bundle $B \stackrel{\varpi}{\longrightarrow} M$ via the map $\pi: A \rightarrow M$, while the vector bundle $A \oplus B \rightarrow B$ is the pullback of the vector bundle $A \stackrel{\pi}{\rightarrow} M$ via the map $\varpi: B \rightarrow M$.

Each section $b \in \Gamma(B)$ determines a derivation $\vec{b}$ of the algebra of smooth functions $C^{\infty}(A)$ through the relations

and

$$
\vec{b}\left(\pi^{*} f\right)=\pi^{*}\left(\varrho_{b} f\right), \quad \forall f \in C^{\infty}(M)
$$

$$
\vec{b}\left(l_{\xi}\right)=l_{\nabla_{b}^{\text {Bott }} \xi}, \quad \forall \xi \in \Gamma\left(A^{\vee}\right),
$$

where $l_{\xi}$ denotes the fiberwise linear function $A \ni a \mapsto\langle\xi \mid a\rangle \in \mathbb{R}$ on $A$.

The vector bundle $A \oplus B \rightarrow A$, whose space of sections is naturally identified to $C^{\infty}(A) \otimes_{C^{\infty}(M)} \Gamma(B)$, admits a natural Lie algebroid structure with anchor map

$$
C^{\infty}(A) \otimes_{C^{\infty}(M)} \Gamma(B) \ni g \otimes b \mapsto g \cdot \vec{b} \in \mathfrak{X}(A), \quad \forall g \in C^{\infty}(A), b \in \Gamma(B)
$$

and Lie bracket

$\left[g_{1} \otimes b_{1}, g_{2} \otimes b_{2}\right]=g_{1} g_{2} \otimes\left[b_{1}, b_{2}\right]+g_{1} \cdot \overrightarrow{b_{1}}\left(g_{2}\right) \otimes b_{2}-g_{2} \cdot \overrightarrow{b_{2}}\left(g_{1}\right) \otimes b_{1}, \quad \forall g_{1}, g_{2} \in C^{\infty}(A), b_{1}, b_{2} \in \Gamma(B)$.

Similarly, the vector bundle $A \oplus B \rightarrow B$ admits a natural Lie algebroid structure. These two Lie algebroid structures on $A \oplus B$ are known to be compatible in the following sense:

Lemma 4.1 (Mackenzie [34]). If $A \bowtie B$ is a matched pair of Lie algebroids, then

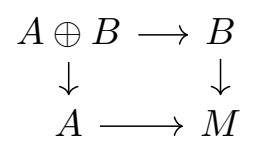

is a double Lie algebroid.

According to Voronov [52], any double Lie algebroid induces a pair of dg Lie algebroids. As an immediate consequence, we have the following

Corollary 4.2. If $A \bowtie B$ is a matched pair of Lie algebroids, then $\left(A[1] \oplus B, d_{A}^{\text {Bott }}\right)$ is a dg Lie algebroid over $\left(A[1], d_{A}\right)$.

Here the dg manifold structures on $\left(A[1] \oplus B, d_{A}^{\text {Bott }}\right)$ and $\left(A[1], d_{A}\right)$ are induced, respectively, from the Lie algebroid structures on $A \oplus B \rightarrow B$ and $A \rightarrow M$ according to Vauntrob's theorem [47] — see Example 2.1. In what follows, we write $\mathcal{B}$ to denote the $\operatorname{dg}$ manifold $\left(A[1] \oplus B, d_{A}^{\text {Bott }}\right)$ and $A[1]$ to denote the dg manifold $\left(A[1], d_{A}\right)$.

The space of sections of the $\operatorname{dg}$ Lie algebroid $\mathcal{B} \rightarrow A[1]$ can be identified naturally with $\Gamma\left(\Lambda^{\bullet} A^{\vee} \otimes B\right)$. Then, the Lie bracket on $\Gamma\left(\Lambda^{\bullet} A^{\vee} \otimes B\right)$ is

$$
\left[\xi_{1} \otimes b_{1}, \xi_{2} \otimes b_{2}\right]=\xi_{1} \wedge \xi_{2} \otimes\left[b_{1}, b_{2}\right]+\xi_{1} \wedge\left(\nabla_{b_{1}}^{\text {Bott }} \xi_{2}\right) \otimes b_{2}-\left(\nabla_{b_{2}}^{\text {Bott }} \xi_{1}\right) \wedge \xi_{2} \otimes b_{1}
$$

for all $\xi_{1}, \xi_{2} \in \Gamma\left(\Lambda^{\bullet} A^{\vee}\right)$ and $b_{1}, b_{2} \in \Gamma(B)$, while the anchor map

$$
\Gamma\left(\Lambda^{\bullet} A^{\vee} \otimes B\right) \stackrel{\bar{\varrho}}{\rightarrow} \operatorname{Der}\left(\Gamma\left(\Lambda^{\bullet} A^{\vee}\right)\right)
$$


is characterized by the relation

$$
\bar{\varrho}_{\xi \otimes b}(\eta)=\xi \wedge \nabla_{b}^{\mathrm{Bott}} \eta,
$$

for all $\xi, \eta \in \Gamma\left(\Lambda^{\bullet} A^{\vee}\right)$ and $b \in \Gamma(B)$. Finally, the differential on the space of sections of $\mathcal{B} \rightarrow A[1]$ induced by the homological vector fields on $\mathcal{B}$ and $A[1]$ is simply the Chevalley-Eilenberg differential

$$
d_{A}^{\text {Bott }}: \Gamma\left(\Lambda^{\bullet} A^{\vee} \otimes B\right) \rightarrow \Gamma\left(\Lambda^{\bullet+1} A^{\vee} \otimes B\right)
$$

corresponding to the Bott representation of $A$ on $B$.

4.2. Fedosov dg manifolds associated with matched pairs. The identification $L=A \oplus B$ induces a decomposition

$$
\Gamma\left(\Lambda^{n} L^{\vee}\right)=\bigoplus_{\substack{p+q=n \\ p, q \geqslant 0}} \Gamma\left(\Lambda^{p} A^{\vee} \otimes \Lambda^{q} B^{\vee}\right), \quad n \geqslant 0 .
$$

Denote by

$$
d_{L}: \Gamma\left(\Lambda^{\bullet} L^{\vee}\right) \rightarrow \Gamma\left(\Lambda^{\bullet} L^{\vee}\right)
$$

the Chevalley-Eilenberg differential of the Lie algebroid $L$ for cochains with trivial coefficients.

Since $A$ and $B$ play symmetric roles as a pair of complementary Lie subalgebroids of the Lie algebroid $L$, we have a pair of Bott connections: the Bott $A$-connection on $B$ and the Bott $B$-connection on $A$, both denoted by $\nabla^{\text {Bott }}$ by abuse of notations. Denote by

$$
d_{A}^{\text {Bott }}: \Gamma\left(\Lambda^{\bullet} A^{\vee} \otimes \Lambda^{\diamond} B^{\vee}\right) \rightarrow \Gamma\left(\Lambda^{\bullet+1} A^{\vee} \otimes \Lambda^{\diamond} B^{\vee}\right)
$$

the Chevalley-Eilenberg differential of the Lie algebroid $A$ for cochains with coefficients in the $A$ module $\Lambda B^{\vee}$ - the implicit flat $A$-connection $\nabla^{\text {Bott }}$ on $\Lambda B^{\vee}$ is induced from the Bott $A$-connection on $B$. Similarly, denote by

$$
d_{B}^{\text {Bott }}: \Gamma\left(\Lambda^{\bullet} A^{\vee} \otimes \Lambda^{\diamond} B^{\vee}\right) \rightarrow \Gamma\left(\Lambda^{\bullet} A^{\vee} \otimes \Lambda^{\diamond+1} B^{\vee}\right)
$$

the Chevalley-Eilenberg differential of the Lie algebroid $B$ for cochains with coefficients in the $B$ module $\Lambda A^{\vee}$ - the implicit flat $B$-connection $\nabla^{\text {Bott }}$ on $\Lambda A^{\vee}$ is induced from the Bott $B$-connection on $A$.

In order to describe the Fedosov dg manifold arising from the Lie pair $(L, A)$, we need to choose a torsion-free $L$-connection $\nabla$ on $B$. Such an $L$-connection on $B$ is completely determined by, and in fact equivalent to, a torsion-free $B$-connection $\nabla^{1,0}$ on $B$.

The following lemma can be verified by a direct computation.

Lemma 4.3. For a matched pair $L=A \bowtie B$, having identified $\Lambda L^{\vee}$ with $\Lambda A^{\vee} \otimes \Lambda B^{\vee}$ as in (47), we have

$$
d_{L}=d_{A}^{\text {Bott }}+d_{B}^{\text {Bott }} .
$$

Furthermore, the covariant differential $d_{L}^{\nabla}$ appearing in Theorem 2.11 decomposes as the sum

$$
d_{L}^{\nabla}=d_{A}^{\text {Bott }}+d_{B}^{\nabla^{1,0}}
$$

of

$$
d_{A}^{\text {Bott }}: \Gamma\left(\Lambda^{\bullet} A^{\vee} \otimes \Lambda^{\diamond} B^{\vee} \otimes \hat{S}\left(B^{\vee}\right)\right) \rightarrow \Gamma\left(\Lambda^{\bullet+1} A^{\vee} \otimes \Lambda^{\diamond} B^{\vee} \otimes \hat{S}\left(B^{\vee}\right)\right)
$$

and

$$
d_{B}^{\nabla^{1,0}}: \Gamma\left(\Lambda^{\bullet} A^{\vee} \otimes \Lambda^{\diamond} B^{\vee} \otimes \hat{S}\left(B^{\vee}\right)\right) \rightarrow \Gamma\left(\Lambda^{\bullet} A^{\vee} \otimes \Lambda^{\diamond+1} B^{\vee} \otimes \hat{S}\left(B^{\vee}\right)\right)
$$


Similarly, the 1-form $X^{\nabla} \in \Gamma\left(L^{\vee} \otimes \hat{S}^{\geqslant 2}\left(B^{\vee}\right) \otimes B\right)$ valued in formal vertical vector fields on $B$ constructed in Theorem 2.11 decomposes as the sum

$$
X^{\nabla}=X^{1,0}+X^{0,1}
$$

of two formal vertical vector fields

$$
X^{0,1} \in \Gamma\left(A^{\vee} \otimes \hat{S}^{\geqslant 2}\left(B^{\vee}\right) \otimes B\right) \quad \text { and } \quad X^{1,0} \in \Gamma\left(B^{\vee} \otimes \hat{S}^{\geqslant 2}\left(B^{\vee}\right) \otimes B\right) .
$$

The following lemma is quite obvious - see [3, Section 5].

Lemma 4.4. Given a matched pair $L=A \bowtie B$, consider the Lie pair $(L, A)$ with the splitting identifying $B$ to a Lie subalgebroid of $L$ complementary to $A$ and choose a torsion-free $B$-connection $\nabla^{1,0}$ on B. Then, the Fedosov homological vector field $Q$ constructed in Theorem 2.11 is the sum $Q=Q^{1,0}+Q^{0,1}$ of the pair of operators

$$
Q^{1,0}: \Gamma\left(\Lambda^{\bullet} A^{\vee} \otimes \Lambda^{\diamond} B^{\vee} \otimes \hat{S}\left(B^{\vee}\right)\right) \rightarrow \Gamma\left(\Lambda^{\bullet} A^{\vee} \otimes \Lambda^{\diamond+1} B^{\vee} \otimes \hat{S}\left(B^{\vee}\right)\right)
$$

and

$$
Q^{0,1}: \Gamma\left(\Lambda^{\bullet} A^{\vee} \otimes \Lambda^{\diamond} B^{\vee} \otimes \hat{S}\left(B^{\vee}\right)\right) \rightarrow \Gamma\left(\Lambda^{\bullet+1} A^{\vee} \otimes \Lambda^{\diamond} B^{\vee} \otimes \hat{S}\left(B^{\vee}\right)\right)
$$

defined by the relations

$$
Q^{1,0}=-\delta+d_{B}^{\nabla^{1,0}}+X^{1,0} \quad \text { and } \quad Q^{0,1}=d_{A}^{\text {Bott }}+X^{0,1}
$$

and satisfying the relations

$$
Q^{1,0} \circ Q^{1,0}=0, \quad Q^{0,1} \circ Q^{0,1}=0, \quad \text { and } \quad Q^{0,1} \circ Q^{1,0}+Q^{1,0} \circ Q^{0,1}=0 .
$$

We now give a more detailed description of the operators $Q^{1,0}$ and $Q^{0,1}$, which will be needed later on. Consider (i) the isomorphism of left $R$-modules pbw $: \Gamma(S B) \rightarrow \frac{\mathcal{U}(L)}{\mathcal{U}(L) \Gamma(A)}$ arising from the Lie pair $(L, A)$ and the $L$-connection $\nabla$ on $B$, (ii) the isomorphism of left $R$-modules $\widetilde{p b w}: \Gamma(S B) \rightarrow \mathcal{U}(B)$ arising from the Lie pair $(B, 0)$ and the $B$-connection $\nabla^{1,0}$ on $B$ - see Equation $(27)$ - and (iii) the natural isomorphism of left $R$-modules

$$
\frac{\mathcal{U}(L)}{\mathcal{U}(L) \Gamma(A)} \stackrel{\cong}{\longrightarrow} \mathcal{U}(B) \text {. }
$$

The following lemma can be verified easily by applying the PBW iteration formula in $[28,29]$ — see also $[45, \S 3.4]$.

Lemma 4.5. Given a matched pair $L=A \bowtie B$, the diagram

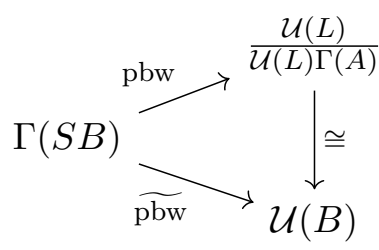

commutes.

The flat $L$-connection $\nabla^{\xi}$ on $S B$ defined by Equation (28) gives rise to a flat $A$-connection on $S B$ :

$$
\widetilde{\nabla}_{a}^{\xi}(s)=\nabla_{i(a)}^{\xi} s=\operatorname{pbw}^{-1}(i(a) \cdot \operatorname{pbw}(s))={\widetilde{\mathrm{pbw}^{-1}}}^{-1}(a \star \operatorname{pbw}(s))
$$

and a flat $B$-connection on $S B$ :

$$
\widetilde{\nabla}_{b}^{\xi}(s)=\nabla_{j(b)}^{\xi} s=\operatorname{pbw}^{-1}(j(b) \cdot \operatorname{pbw}(s))=\widetilde{\mathrm{pbw}}^{-1}(b \cdot \widetilde{\mathrm{pbw}}(s)) .
$$

Here $a \in \Gamma(A), b \in \Gamma(B), s \in \Gamma(S B)$. The symbol - appearing in the r.h.s. of Equation (50) denotes the multiplication in $\mathcal{U}(B)$, while the symbol $\star$ appearing in the r.h.s. of Equation (49) denotes the 
action of $A$ on $\mathcal{U}(B)$ induced by the multiplication in $\mathcal{U}(L)$ and the natural identification of $\frac{\mathcal{U}(L)}{\mathcal{U}(L) \Gamma(A)}$ with $\mathcal{U}(B)$.

According to Theorem 2.13 (see also [45, Theorem 4.7]), the homological vector field $Q$ on the Fedosov dg manifold $L[1] \oplus B$ is the Chevalley-Eilenberg differential (29) corresponding to the flat $L$-connection on $\hat{S}\left(B^{\vee}\right)$ dual to the flat $L$-connection $\nabla^{\xi}$ on $S B$ defined by Equation (28). Therefore, as an immediate consequence of Lemma 4.3, we obtain

Corollary 4.6. Under the assumptions of Lemma 4.4,

(1) the operator $Q^{1,0}$ coincides with the Chevalley-Eilenberg differential of the Lie algebroid $B$ for cochains with coefficients in the B-module $\Lambda A^{\vee} \otimes \hat{S}\left(B^{\vee}\right)$ with the B-representation

$$
\nabla_{b}(\alpha \otimes \varsigma)=\nabla_{b}^{\text {Bott }} \alpha \otimes \varsigma+\alpha \otimes \widetilde{\nabla}_{b}^{\xi} \varsigma,
$$

for all $\alpha \otimes \varsigma \in \Gamma\left(\Lambda A^{\vee} \otimes \hat{S}\left(B^{\vee}\right)\right)$.

(2) and the operator $Q^{0,1}$ coincides with the Chevalley-Eilenberg differential of the Lie algebroid $A$ for cochains with coefficients in the A-module $\Lambda B^{\vee} \otimes \hat{S}\left(B^{\vee}\right)$ with the A-representation

$$
\nabla_{a}(\beta \otimes \varsigma)=\nabla_{a}^{\text {Bott }} \beta \otimes \varsigma+\beta \otimes \widetilde{\nabla}_{a}^{\xi} \varsigma .
$$

for all $\beta \otimes s \in \Gamma\left(\Lambda B^{\vee} \otimes \hat{S}\left(B^{\vee}\right)\right)$.

Here $\widetilde{\nabla}_{a}^{\xi}$ and $\widetilde{\nabla}_{b}^{\xi}$ are the flat connections introduced in Equations (49) and (50), respectively.

Restricting the operator $Q^{0,1}$ to $\Gamma\left(\Lambda^{\bullet} A^{\vee} \otimes \hat{S}\left(B^{\vee}\right)\right)$ determines a derivation $Q^{0,1}$ of $\Gamma\left(\Lambda^{\bullet} A^{\vee} \otimes \hat{S} B^{\vee}\right)$ of degree +1 such that $Q^{0,1} \circ Q^{0,1}=0$. In other words, $Q^{0,1}$ is a homological vector field on the graded manifold $A[1] \oplus B$. Hence $\left(A[1] \oplus B, Q^{0,1}\right)$ is a dg manifold. Corollary 4.6 implies that $\left(A[1] \oplus B, Q^{0,1}\right)$ is indeed an instance of the Kapranov dg manifolds investigated in [28, 29].

Remark 4.7. Given a complex manifold $X$, let $A=T_{X}^{0,1}$ and $B=T_{X}^{1,0}$. Then $T_{X}^{\mathbb{C}}=A \bowtie B$ is a matched pair of Lie algebroids over $\mathbb{C}$. The Bott $T_{X}^{0,1}$-connection on $T_{X}^{1,0}$ encodes the holomorphic vector bundle structure of $T_{X}^{1,0}$; the (local) sections of $T_{X}^{1,0}$ which are flat w.r.t. the $T_{X}^{0,1}$-connection are precisely the (local) holomorphic sections of $T_{X}^{1,0}$. In other words, the Chevalley-Eilenberg differential associated with the Bott $T_{X}^{0,1}$-connection on $T_{X}^{1,0}$ is the Dolbeault operator

$$
\bar{\partial}: \Omega^{0, \bullet}\left(X, T_{X}^{1,0}\right) \rightarrow \Omega^{0, \bullet+1}\left(X, T_{X}^{1,0}\right) .
$$

Similarly, the Chevalley-Eilenberg differential associated with the Bott $T_{X}^{1,0}$-connection on $T_{X}^{0,1}$ is the complex conjugate operator

$$
\partial: \Omega^{\bullet, 0}\left(X, T_{X}^{0,1}\right) \rightarrow \Omega^{\bullet+1,0}\left(X, T_{X}^{0,1}\right)
$$

To construct a Fedosov dg manifold corresponding to the matched pair $\left(T_{X}^{0,1}, T_{X}^{1,0}\right)$, we need a torsionfree $T_{X}^{\mathbb{C}}$-connection $\nabla$ on $T_{X}^{1,0}$, which is necessarily the sum $\nabla=\bar{\partial}+\nabla^{1,0}$ of the Dolbeault operator and a torsion-free $T_{X}^{1,0}$-connection $\nabla^{1,0}$ on $T_{X}^{1,0}-$ more precisely, we have $d^{\nabla}=\bar{\partial}+d^{\nabla^{1,0}}$. The graded manifold underlying this Fedosov dg manifold is $T_{X}^{\mathbb{C}}[1] \oplus T_{X}^{1,0}$ with the algebra of functions

$$
C^{\infty}\left(T_{X}^{\mathbb{C}}[1] \oplus T_{X}^{1,0}\right) \cong \bigoplus_{p \geqslant 0, q \geqslant 0} \Omega^{p, q}\left(X, \hat{S}\left(T_{X}^{1,0}\right)^{\vee}\right) .
$$

Its homological vector field decomposes as the sum

$$
Q=Q^{1,0}+Q^{0,1}
$$

of two operators

$$
Q^{1,0}: \Omega^{p, q}\left(X, \hat{S}\left(T_{X}^{1,0}\right)^{\vee}\right) \rightarrow \Omega^{p+1, q}\left(X, \hat{S}\left(T_{X}^{1,0}\right)^{\vee}\right)
$$

and

$$
Q^{0,1}: \Omega^{p, q}\left(X, \hat{S}\left(T_{X}^{1,0}\right)^{\vee}\right) \rightarrow \Omega^{p, q+1}\left(X, \hat{S}\left(T_{X}^{1,0}\right)^{\vee}\right)
$$


given by

$$
Q^{1,0}=-\delta+d^{\nabla^{1,0}}+X^{1,0} \quad \text { and } \quad Q^{0,1}=\bar{\partial}+X^{0,1}
$$

with

$$
X^{1,0} \in \Omega^{1,0}\left(X, \hat{S}^{\geqslant 2}\left(T_{X}^{1,0}\right)^{\vee} \otimes T_{X}^{1,0}\right) \quad \text { and } \quad X^{0,1} \in \Omega^{0,1}\left(X, \hat{S}^{\geqslant 2}\left(T_{X}^{1,0}\right)^{\vee} \otimes T_{X}^{1,0}\right) .
$$

Here $\delta$ is the usual Koszul operator and $d^{\nabla^{1,0}}$ is the Chevalley-Eilenberg differential associated with the $T_{X}^{1,0}$-connection $\partial \otimes \mathrm{id}+\mathrm{id} \otimes \nabla^{1,0}$ on $\Lambda^{q}\left(T_{X}^{0,1}\right)^{\vee} \otimes \hat{S}\left(T_{X}^{1,0}\right)^{\vee}$.

Restricting to $p=0$ in (51), we obtain a derivation $Q^{0,1}$ of degree +1 of the algebra $\Omega^{0, \bullet}\left(X, \hat{S}\left(T_{X}^{1,0}\right)^{\vee}\right)$ satisfying $Q^{0,1} \circ Q^{0,1}=0$. Therefore, $Q^{0,1}$ induces a $L_{\infty}[1]$ algebra structure (see [23]) on $\Omega^{0, \bullet}\left(X, T_{X}^{1,0}\right)$, and $\left(T_{X}^{0,1}[1] \oplus T_{X}^{1,0}, Q^{0,1}\right)$ is a Kapranov dg manifold - see [29, Section 5.5]. If the complex manifold $X$ admits a Kähler metric, there is a canonical torsion-free flat $T_{X}^{1,0}$-connection $\nabla^{1,0}$ on $T_{X}^{1,0}$ induced by the Levi-Civita connection on $T_{X}$. In that case, Kapranov obtained an explicit formula for the $L_{\infty}[1]$ algebra structure on $\Omega^{0, \bullet}\left(X, T_{X}^{1,0}\right)$ - see [23, Theorem 2.6]. Such $L_{\infty}$ [1] algebras played an important role in Kapranov's investigation [23] of Atiyah classes and Rozansky-Witten invariants - see also [29, 28, 51].

4.3. Polyvector fields associated with matched pairs. According to Proposition 2.7, the dg Lie algebroid structure on $\mathcal{B} \rightarrow A[1]$ induces a differential Gerstenhaber algebra structure on $\Gamma\left(\Lambda^{\bullet+1} \mathcal{B}\right) \cong \Gamma\left(\Lambda^{\bullet} A^{\vee} \otimes \Lambda^{\bullet+1} B\right)$. Its differential is the Chevalley-Eilenberg differential

$$
d_{A}^{\text {Bott }}: \Gamma\left(\Lambda^{k} A^{\vee} \otimes \Lambda^{p+1} B\right) \rightarrow \Gamma\left(\Lambda^{k+1} A^{\vee} \otimes \Lambda^{p+1} B\right)
$$

corresponding to the Bott representation of $A$ on $\Lambda B$; its associative multiplication is the wedge product

$$
\wedge: \Gamma\left(\Lambda^{k} A^{\vee} \otimes \Lambda^{p+1} B\right) \otimes \Gamma\left(\Lambda^{l} A^{\vee} \otimes \Lambda^{q+1} B\right) \rightarrow \Gamma\left(\Lambda^{k+l} A^{\vee} \otimes \Lambda^{(p+q+1)+1} B\right) ;
$$

and its Lie bracket

$$
[-,-]: \Gamma\left(\Lambda^{k} A^{\vee} \otimes \Lambda^{p+1} B\right) \otimes \Gamma\left(\Lambda^{l} A^{\vee} \otimes \Lambda^{q+1} B\right) \rightarrow \Gamma\left(\Lambda^{k+l} A^{\vee} \otimes \Lambda^{p+q+1} B\right)
$$

is the Schouten bracket of the $\operatorname{dg}$ Lie algebroid $\mathcal{B} \rightarrow A[1]$ extending the Lie bracket (45) by way of the Leibniz rule and the anchor map (46).

Applying Proposition 2.7 to the dg Lie algebroid $\mathcal{B} \rightarrow A[1]$, we obtain the following

Proposition 4.8. Let $A \bowtie B$ be a matched pair of Lie algebroids.

(1) When endowed with the differential $d_{A}^{\text {Bott }}$ (52); the associative multiplication (53); and the Lie bracket (54), tot $\Gamma\left(\Lambda^{\bullet} A^{\vee} \otimes \Lambda^{\diamond+1} B\right)$ is a differential Gerstenhaber algebra, whence a dgla.

(2) When endowed with the wedge product (53) and the Schouten bracket (54), the cohomology $\mathbb{H}_{\mathrm{CE}}^{\bullet}\left(A, \Lambda^{\bullet+1} B\right)$ is a Gerstenhaber algebra.

The following theorem is the first main result of the present section.

Theorem 4.9. Let $L=A \bowtie B$ be a matched pair and let $\nabla$ be a torsion-free $L$-connection on $B$. Then the $L_{\infty}$ algebra tot $\left(\Gamma\left(\Lambda^{\bullet} A^{\vee}\right) \otimes_{R} \mathcal{T}_{\text {poly }}^{\diamond}\right)$ and the Gerstenhaber algebra $\mathbb{H}_{\mathrm{CE}}^{\bullet}\left(A, \mathcal{T}_{\text {poly }}\right)$ of Proposition 3.9 coincide with the dgla tot $\Gamma\left(\Lambda^{\bullet} A^{\vee} \otimes \Lambda^{\diamond+1} B\right)$ and the Gerstenhaber algebra $\mathbb{H}_{\mathrm{CE}}^{\bullet}\left(A, \Lambda^{\diamond+1} B\right)$ of Proposition 4.8, respectively.

Theorem 4.9 is a direct consequence of [2, Proposition 4.9] — see also [2, Theorem 4.20]. However, for the sake of completeness, we proceed to outline a direct proof.

Denote by $\mathfrak{X}_{\text {ver }}(B)$ the space of formal vertical vector fields on the vector bundle $B \rightarrow M$. We have the natural identification

$$
\mathfrak{X}_{\mathrm{ver}}(B) \cong \Gamma\left(\hat{S}\left(B^{\vee}\right) \otimes B\right)
$$


Since $\mathcal{T}_{\text {poly }}^{-1}=C^{\infty}(M) ; \mathcal{T}_{\text {poly }}^{0}=\Gamma(B) ; \mathscr{T}_{\text {poly }}^{-1} \cong \Gamma\left(\hat{S}\left(B^{\vee}\right)\right) ;$ and $\mathscr{T}_{\text {poly }}^{0} \cong \mathfrak{X}_{\text {ver }}(B)$, specializing the contraction of Corollary 3.5 in the cases where $k=-1$ and $k=0$ yields a pair of contractions:

$$
\left(\Gamma\left(\Lambda^{\bullet} A^{\vee}\right), d_{A}\right) \underset{\tau}{\stackrel{\breve{\tau}}{\rightleftarrows}}\left(\Gamma\left(\Lambda^{\bullet} L^{\vee} \otimes \hat{S}\left(B^{\vee}\right)\right), Q\right) \supset \breve{h}
$$

and

$$
\left(\Gamma\left(\Lambda^{\bullet} A^{\vee} \otimes B\right), d_{A}^{\text {Bott }}\right) \underset{\sigma_{\natural}}{\stackrel{\breve{\tau}_{\natural}}{\rightleftarrows}}\left(\Gamma\left(\Lambda^{\bullet} L^{\vee}\right) \otimes_{R} \mathfrak{X}_{\mathrm{ver}}(B), \mathcal{L}_{Q}\right) \curlyvee \breve{h}_{\natural}
$$

Note that $\left(\Gamma\left(\Lambda^{\bullet} A^{\vee} \otimes B\right), d_{A}^{\text {Bott }}\right)$ is a dg Lie-Rinehart algebra over the dg ring $\left(\Gamma\left(\Lambda^{\bullet} A^{\vee}\right), d_{A}\right)$ while $\left(\Gamma\left(\Lambda^{\bullet} L^{\vee}\right) \otimes_{R} \mathfrak{X}_{\text {ver }}(B), \mathcal{L}_{Q}\right)$ is a dg Lie-Rinehart algebra over the dg $\operatorname{ring}\left(\Gamma\left(\Lambda^{\bullet} L^{\vee} \otimes \hat{S}\left(B^{\vee}\right)\right), Q\right)$.

Proposition 4.10. The pair of maps $\breve{\tau}$ and $\breve{\tau}_{\natural}$ in the contractions (55) and (56) constitutes a morphism of $d g$ Lie-Rinehart algebras from $\left(\Gamma\left(\Lambda^{\bullet} A^{\vee} \otimes B\right), d_{A}^{\text {Bott }}\right)$ to $\left(\Gamma\left(\Lambda^{\bullet} L^{\vee}\right) \otimes_{R} \mathfrak{X}_{\mathrm{ver}}(B), \mathcal{L}_{Q}\right)$.

Recall that $\breve{\tau}_{\natural}=\sum_{l=0}^{\infty}\left(h_{\natural} \mathcal{L}_{\rho}\right)^{l} \tau_{\natural}$ where

$$
\rho=d_{L}^{\nabla}+X^{\nabla}=\left(d_{A}^{\text {Bott }}+d_{B}^{\nabla^{1,0}}\right)+\left(X^{0,1}+X^{1,0}\right),
$$

since $L=A \oplus B$ is a matched pair. It is simple to see that

$$
\mathcal{L}_{d_{A}^{\text {Bott }}+X^{0,1}}\left(\Gamma\left(\Lambda^{\bullet} A^{\vee} \otimes \Lambda^{0} B^{\vee}\right) \otimes_{R} \mathfrak{X}_{\text {ver }}(B)\right) \subset \Gamma\left(\Lambda^{\bullet+1} A^{\vee} \otimes \Lambda^{0} B^{\vee}\right) \otimes_{R} \mathfrak{X}_{\text {ver }}(B) \subset \operatorname{ker} h_{\natural}
$$

and

$$
\mathcal{L}_{d_{B}^{\nabla 1,0}+X^{1,0}}\left(\Gamma\left(\Lambda^{\bullet} A^{\vee} \otimes \Lambda^{0} B^{\vee}\right) \otimes_{R} \mathfrak{X}_{\mathrm{ver}}(B)\right) \subset \Gamma\left(\Lambda^{\bullet} A^{\vee} \otimes \Lambda^{1} B^{\vee}\right) \otimes_{R} \mathfrak{X}_{\mathrm{ver}}(B) .
$$

Therefore, the operator $h_{\natural} \mathcal{L}_{\rho}$ stabilizes $\Gamma\left(\Lambda^{\bullet} A^{\vee} \otimes \Lambda^{0} B^{\vee}\right) \otimes_{R} \mathfrak{X}_{\text {ver }}(B)$ and we can conclude that

$$
\breve{\tau}_{\mathrm{\natural}}\left(\Gamma\left(\Lambda^{\bullet} A^{\vee} \otimes B\right)\right) \subset \Gamma\left(\Lambda^{\bullet} A^{\vee} \otimes \Lambda^{0} B^{\vee}\right) \otimes_{R} \mathfrak{X}_{\mathrm{ver}}(B) .
$$

Since $\breve{\tau}_{\natural}$ is a cochain map, we have $\mathcal{L}_{Q} \circ \breve{\tau}_{\natural}=\breve{\tau}_{\natural} \circ d_{A}^{\text {Bott }}$, and it follows that

$$
\mathcal{L}_{Q^{0,1}} \circ \breve{\tau}_{\natural}=\breve{\tau}_{\natural} \circ d_{A}^{\text {Bott }} \quad \text { and } \quad \mathcal{L}_{Q^{1,0}} \circ \breve{\tau}_{\natural}=0,
$$

where $Q^{0,1}$ and $Q^{1,0}$ are the vector fields defined in Lemma 4.4 .

Proof of Proposition 4.10. It suffices to verify that the pair of maps $\breve{\tau}$ and $\breve{\tau}_{\natural}$ in the contractions (55) and (56) satisfy the identities

$$
\begin{gathered}
{\left[\breve{\tau}_{\natural}(\xi \otimes b), \breve{\tau}_{\natural}(\eta \otimes c)\right]=\breve{\tau}_{\natural}[\xi \otimes b, \eta \otimes c]} \\
{\left[\breve{\tau}_{\natural}(\xi \otimes b), \breve{\tau}(\eta)\right]=\breve{\tau}[\xi \otimes b, \eta]} \\
\breve{\tau}(\eta) \cdot \breve{\tau}_{\natural}(\xi \otimes b)=\breve{\tau}_{\natural}(\eta \cdot \xi \otimes b)
\end{gathered}
$$

for all $\xi, \eta \in \Gamma\left(\Lambda^{\bullet} A^{\vee}\right)$ and $b, c \in \Gamma(B)$. In Equations (60) and (61), the brackets on the r.h.s. are Schouten brackets of polyvector fields on the dg Lie algebroid $\mathcal{B}$, while the brackets on the 1.h.s. are Schouten brackets of polyvector fields on the dg Lie algebroid $\mathcal{F}$.

Consider

$$
\mathscr{Y}=\left[\breve{\tau}_{\natural}(\xi \otimes b), \breve{\tau}_{\natural}(\eta \otimes c)\right] .
$$

It follows from (58) that $\mathscr{Y} \in \Gamma\left(\Lambda^{\bullet} A^{\vee} \otimes \Lambda^{0} B^{\vee}\right) \otimes_{R} \mathfrak{X}_{\text {ver }}(B)$ and thence $h_{\natural}(\mathscr{Y})=0$. Since $Q^{0,1}=d_{A}^{\text {Bott }}+X^{0,1}$, according to (57) we also get $h_{\natural} \mathcal{L}_{Q^{0,1}}(\mathscr{Y})=0$. Furthermore, from Equation (59), we obtain

$$
\begin{aligned}
\mathcal{L}_{Q^{1,0}} \mathscr{Y}=\mathcal{L}_{Q^{1,0}}\left(\left[\breve{\tau}_{\natural}(\xi \otimes b), \breve{\tau}_{\natural}(\eta \otimes c)\right]\right) \\
\quad=\left[\mathcal{L}_{Q^{1,0}}\left(\breve{\tau}_{\natural}(\xi \otimes b)\right), \breve{\tau}_{\natural}(\eta \otimes c)\right] \pm\left[\breve{\tau}_{\natural}(\xi \otimes b), \mathcal{L}_{Q^{1,0}}\left(\breve{\tau}_{\natural}(\eta \otimes c)\right)\right]=0 .
\end{aligned}
$$

Therefore, we conclude that

$$
h_{\natural} \mathcal{L}_{Q}(\mathscr{Y})=h_{\natural} \mathcal{L}_{Q^{0,1}}(\mathscr{Y})+h_{\natural} \mathcal{L}_{Q^{1,0}}(\mathscr{Y})=0 .
$$


From the definitions (34) and (37) (see also [2, Lemma 4.13]) of $\sigma_{\natural}$ and $\breve{\tau}_{\natural}$, we obtain

$$
\sigma_{\natural}(\mathscr{Y})=\sigma_{\natural}\left[\breve{\tau}_{\natural}(\xi \otimes b), \breve{\tau}_{\natural}(\eta \otimes c)\right]=[\xi \otimes b, \eta \otimes c] .
$$

Since $h_{\natural}(\mathscr{Y})=0 ; h_{\natural} \mathcal{L}_{Q}(\mathscr{Y})=0$; and $\sigma_{\natural}(\mathscr{Y})=[\xi \otimes b, \eta \otimes c]$, it follows from Proposition 3.8 that

$$
\mathscr{Y}=\breve{\tau}_{\natural}([\xi \otimes b, \eta \otimes c]) \text {. }
$$

Identity (60) is thus established. Identities (61) and (62) can be verified in a similar fashion.

We are now ready to prove Theorem 4.9 .

Proof of Theorem 4.9. Since $L=A \bowtie B$ is a matched pair, the cochain complex on the l.h.s. of (36) in Proposition 3.4 is tot $\Gamma\left(\Lambda^{\bullet} A^{\vee} \otimes \Lambda^{\diamond+1} B\right)$. It suffices to prove that the injection $\breve{\tau}_{\natural}$ in (36) is a morphism of Lie algebras, where the Lie bracket on tot $\Gamma\left(\Lambda^{\bullet} A^{\vee} \otimes \Lambda^{\diamond+1} B\right)$ is as in Proposition 4.8. This follows immediately from Proposition 4.10 and the fact that $\breve{\tau}_{\natural}$ respects wedge products by virtue of Proposition 3.4.

4.4. Polydifferential operators associated with matched pairs. We now turn to the study of polydifferential operators.

Recall that the universal enveloping algebra $\mathcal{U}(\mathcal{B})$ of the $\mathrm{dg}$ Lie algebroid $\mathcal{B} \rightarrow A[1]$ is a dg Hopf algebroid over the dgca $C^{\infty}(A[1])=\Gamma\left(\Lambda^{\bullet} A^{\vee}\right)$. There is a natural isomorphism of left $\Gamma\left(\Lambda^{\bullet} A^{\vee}\right)$ modules

$$
\mathcal{U}(\mathcal{B}) \cong \Gamma\left(\Lambda^{\bullet} A^{\vee}\right) \otimes_{R} \mathcal{U}(B) .
$$

Consequently, $\Gamma\left(\Lambda^{\bullet} A^{\vee}\right) \otimes_{R} \mathcal{U}(B)$ admits a structure of dg Hopf algebroid over the $\operatorname{dgca}\left(\Gamma\left(\Lambda^{\bullet} A^{\vee}\right), d_{A}\right)$ :

(1) The multiplication is characterized by the relations

$$
\begin{aligned}
(\xi \otimes 1) \cdot(\eta \otimes 1) & =\xi \wedge \eta \otimes 1, & & \forall \xi, \eta \in \Gamma\left(\Lambda^{\bullet} A^{\vee}\right) ; \\
(1 \otimes u) \cdot(1 \otimes v) & =1 \otimes u \cdot v, & & \forall u, v \in \mathcal{U}(B) ; \\
(\xi \otimes 1) \cdot(1 \otimes u) & =\xi \otimes u, & & \forall \xi \in \Gamma\left(\Lambda^{\bullet} A^{\vee}\right), \forall u \in \mathcal{U}(B) ; \\
(1 \otimes b) \cdot(\xi \otimes 1)-(\xi \otimes 1) \cdot(1 \otimes b) & =\left(\nabla_{b}^{\mathrm{Bott}} \xi\right) \otimes 1, & & \forall b \in \Gamma(B), \forall \xi \in \Gamma\left(A^{\vee}\right) .
\end{aligned}
$$

Indeed, the multiplication is defined by the relation

$$
\left(\xi \otimes b_{1} b_{2} \cdots b_{n}\right) \cdot(\eta \otimes u)=\sum_{k=0}^{n} \sum_{\sigma \in \mathfrak{S}_{k}^{n-k}}\left(\xi \wedge \nabla_{b_{\sigma(1)}}^{\mathrm{Bott}} \cdots \nabla_{b_{\sigma(k)}}^{\mathrm{Bott}} \eta\right) \otimes b_{\sigma(k+1)} \cdots b_{\sigma(n)} \cdot u
$$

for all $\xi, \eta \in \Gamma\left(\Lambda^{\bullet} A^{\vee}\right), b_{1}, b_{2}, \ldots, b_{n} \in \Gamma(B)$, and $u \in \mathcal{U}(B)$. Note that the multiplication is well-defined by Equation (64) because the Bott $B$-connection on $A^{\vee}$ is flat.

(2) The source and target maps

$$
\Gamma\left(\Lambda^{\bullet} A^{\vee}\right) \underset{\beta}{\stackrel{\alpha}{\rightleftarrows}} \Gamma\left(\Lambda^{\bullet} A^{\vee}\right) \otimes_{R} \mathcal{U}(B)
$$

are one and the same map: the inclusion $\xi \mapsto \xi \otimes 1$.

(3) The differential is the Chevalley-Eilenberg differential

$$
d_{A}^{\mathcal{U}}: \Gamma\left(\Lambda^{\bullet} A^{\vee}\right) \otimes_{R} \mathcal{U}(B) \rightarrow \Gamma\left(\Lambda^{\bullet+1} A^{\vee}\right) \otimes_{R} \mathcal{U}(B)
$$

of the Lie algebroid $A$ for cochains with coefficients in $\mathcal{U}(B)$. The $A$-module structure on $\mathcal{U}(B)$ follows from the canonical identification (48) - the Lie algebroid $A$ acts on $\mathcal{U}(L)$ by multiplication from the left. 
(4) The comultiplication $\Delta$ is defined by the commutative diagram of left $\Gamma\left(\Lambda^{\bullet} A^{\vee}\right)$-modules

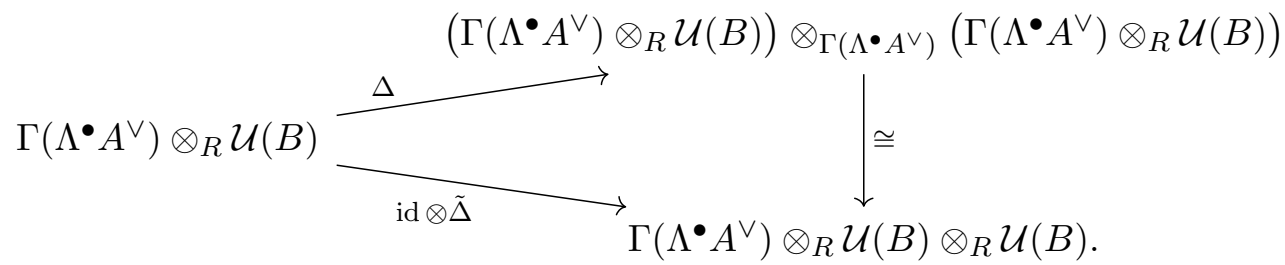

Indeed, it is the $\Gamma\left(\Lambda^{\bullet} A^{\vee}\right)$-linear extension of the comultiplication

$$
\widetilde{\Delta}: \mathcal{U}(B) \rightarrow \mathcal{U}(B) \otimes_{R} \mathcal{U}(B)
$$

of the Hopf algebroid $\mathcal{U}(B)-$ see [55].

(5) The counit map $\varepsilon: \Gamma\left(\Lambda^{\bullet} A^{\vee}\right) \otimes_{R} \mathcal{U}(B) \rightarrow \Gamma\left(\Lambda^{\bullet} A^{\vee}\right)$ is the canonical projection.

From the isomorphism (63), we obtain an isomorphism

$$
(s \mathcal{U}(\mathcal{B}))^{\otimes k+1} \cong \Gamma\left(\Lambda^{\bullet} A^{\vee}\right) \otimes_{R} \mathcal{U}(B)^{\otimes k+1}[-k-1],
$$

which identifies (up to a grading shift) the differential $\mathcal{Q}: s \mathcal{U}(\mathcal{B})^{\otimes k+1} \rightarrow s \mathcal{U}(\mathcal{B})^{\otimes k+1}$ to the ChevalleyEilenberg differential

$$
d_{A}^{\mathcal{U}}: \Gamma\left(\Lambda^{\bullet} A^{\vee}\right) \otimes_{R} \mathcal{U}(B)^{\otimes k+1} \rightarrow \Gamma\left(\Lambda^{\bullet+1} A^{\vee}\right) \otimes_{R} \mathcal{U}(B)^{\otimes k+1} .
$$

Here $\mathcal{U}(B)^{\otimes k+1}$ with $k \geqslant-1$ denotes the tensor product $\mathcal{U}(B) \otimes_{R} \cdots \otimes_{R} \mathcal{U}(B)$ of $(k+1)$-copies of the left $R$-module $\mathcal{U}(B)$, and the $A$-module structure on $\mathcal{U}(B)^{\otimes k+1}$ is the natural extension of the $A$-module structure on $\mathcal{U}(B)$.

The Hochschild coboundary differential (18), the Gerstenhaber bracket (19), and the cup product (22) on $\operatorname{tot}_{\oplus} s \mathcal{U}(\mathcal{B})^{\otimes \bullet+1}$ arising from the dg Lie algebroid $\mathcal{B}$ carry over, through the identification (65), to a Hochschild coboundary differential

$$
\Gamma\left(\Lambda^{\bullet} A^{\vee}\right) \otimes_{R} \mathcal{U}(B)^{\otimes k} \stackrel{\mathfrak{d} \mathscr{H}}{\longrightarrow} \Gamma\left(\Lambda^{\bullet} A^{\vee}\right) \otimes_{R} \mathcal{U}(B)^{\otimes k+1},
$$

a Gerstenhaber bracket

$$
\left(\Gamma\left(\Lambda^{\bullet} A^{\vee}\right) \otimes_{R} \mathcal{U}(B)^{\otimes p+1}\right) \otimes\left(\Gamma\left(\Lambda^{\bullet} A^{\vee}\right) \otimes_{R} \mathcal{U}(B)^{\otimes q+1}\right) \stackrel{\llbracket-,-\rrbracket}{\longrightarrow} \Gamma\left(\Lambda^{\bullet} A^{\vee}\right) \otimes_{R} \mathcal{U}(B)^{\otimes p+q+1},
$$

and a cup product

$$
\left(\Gamma\left(\Lambda^{\bullet} A^{\vee}\right) \otimes_{R} \mathcal{U}(B)^{\otimes p+1}\right) \otimes\left(\Gamma\left(\Lambda^{\bullet} A^{\vee}\right) \otimes_{R} \mathcal{U}(B)^{\otimes q+1}\right) \leftrightharpoons \Gamma\left(\Lambda^{\bullet} A^{\vee}\right) \otimes_{R} \mathcal{U}(B)^{\otimes(p+q+1)+1}
$$

on $\Gamma\left(\Lambda^{\bullet} A^{\vee}\right) \otimes_{R} \mathcal{U}(B)^{\otimes \diamond+1}$.

Note that both the Hochschild coboundary differential and the cup product are $\Gamma\left(\Lambda^{\bullet} A^{\vee}\right)$-linear. That is, we have

$$
\mathfrak{d}_{\mathscr{H}}(\omega \otimes u)=(-1)^{k} \omega \otimes d_{\mathscr{H}}(u)
$$

and

$$
(\omega \otimes u) \smile(\theta \otimes v)=(-1)^{l(p+1)}(\omega \wedge \theta) \otimes(u \otimes v)
$$

for all $\omega \in \Gamma\left(\Lambda^{k} A^{\vee}\right), \theta \in \Gamma\left(\Lambda^{l} A^{\vee}\right), u \in \mathcal{U}(B)^{\otimes p+1}$ and $v \in \mathcal{U}(B)^{\otimes q+1}$. However, the Gerstenhaber bracket (68) is not the obvious extension of the Gerstenhaber bracket on $\mathcal{U}(B)^{\otimes \bullet+1}$ obtained by tensoring with the commutative associative algebra $\Gamma\left(\Lambda^{\bullet} A^{\vee}\right)$. In fact, to write down an explicit formula - which is quite involved - one must use the Bott representation of $B$ on $\Lambda A^{\vee}$.

Applying Proposition 2.8 to the dg Lie algebroid $\mathcal{B} \rightarrow A[1]$, we are led to the following

Proposition 4.11. Let $A \bowtie B$ be a matched pair of Lie algebroids.

(1) When endowed with the differential $d_{A}^{\mathcal{U}}+\mathfrak{d}_{\mathscr{H}}$ (see (66) and (67)) and the Gerstenhaber bracket (68), tot $\left(\Gamma\left(\Lambda^{\bullet} A^{\vee}\right) \otimes_{R} \mathcal{U}(B)^{\otimes \diamond+1}\right)$ is a dgla. 
(2) When endowed with the cup product (69) and the Gerstenhaber bracket (68), the Hochschild cohomology $\mathbb{H}_{\mathrm{CE}}^{\bullet}\left(A, \mathcal{U}(B)^{\otimes \diamond+1}\right)$, i.e. the cohomology of the complex

$$
\left(\operatorname{tot}\left(\Lambda^{\bullet} A^{\vee} \otimes_{R} \mathcal{U}(B)^{\otimes \diamond+1}\right), d_{A}^{\mathcal{U}}+\mathfrak{d}_{\mathscr{H}}\right),
$$

is a Gerstenhaber algebra.

As pointed out in Remark 2.9, on the cochain level, the Gerstenhaber bracket (68) satisfies the graded Leibniz rule with respect to the cup product (69) only up to homotopy. Therefore, tot $\left(\Gamma\left(\Lambda^{\bullet} A^{\vee}\right) \otimes_{R} \mathcal{U}(B)^{\otimes \diamond+1}\right)$ is not a differential Gerstenhaber algebra. Likewise, the cup product is graded commutative only up to homotopy. Again this is reminiscent of ordinary Hochschild cohomology theory of associative algebras [18].

Theorem 4.12 below is the second main result of the present section, the remainder of which is devoted to its proof.

Theorem 4.12. Let $L=A \bowtie B$ be a matched pair and let $\nabla$ be a torsion-free $L$-connection on $B$. Then the $L_{\infty}$ algebra tot $\left(\Gamma\left(\Lambda^{\bullet} A^{\vee}\right) \otimes_{R} \mathcal{D}_{\text {poly }}^{\diamond}\right)$ and the Gerstenhaber algebra $\mathbb{H}_{\mathrm{CE}}^{\bullet}\left(A, \mathcal{D}_{\text {poly }}^{\diamond}\right)$ of Proposition 3.24 coincide with the dgla tot $\left(\Gamma\left(\Lambda^{\bullet} A^{\vee}\right) \otimes_{R} \mathcal{U}(B)^{\diamond+1}\right)$ and the Gerstenhaber algebra $\mathbb{H}_{\mathrm{CE}}^{\bullet}\left(A, \mathcal{U}(B)^{\diamond+1}\right)$ of Proposition 4.11, respectively.

Denote by $\mathfrak{D}_{\text {ver }}(B)$ the algebra of formal vertical differential operators on the vector bundle $B \rightarrow M$. The canonical isomorphism (32), specialized to the case $k=0$, gives the identification

$$
\mathfrak{D}_{\mathrm{ver}}(B) \cong \Gamma\left(\hat{S}\left(B^{\vee}\right) \otimes S B\right) .
$$

Consider the contraction (42) in Corollary 3.19. As an immediate consequence of isomorphism (48), we have $\mathcal{D}_{\text {poly }}^{k} \cong \mathcal{U}(B)^{\otimes k+1}$. We also have isomorphism $(32): \mathscr{D}_{\text {poly }}^{k} \cong \Gamma\left(\hat{S}\left(B^{\vee}\right) \otimes(S B)^{\otimes k+1}\right)$. Specializing Corollary 3.19 to the case $k=0$, we obtain the contraction

$$
\left(\Gamma\left(\Lambda^{\bullet} A^{\vee}\right) \otimes_{R} \mathcal{U}(B), d_{A}^{\mathcal{U}}\right) \underset{\sigma_{\natural}}{\stackrel{\breve{\tau}_{\natural}}{\rightleftarrows}}\left(\Gamma\left(\Lambda^{\bullet} L^{\vee}\right) \otimes_{R} \mathfrak{D}_{\mathrm{ver}}(B), \llbracket Q,-\rrbracket\right) \supset \breve{h}_{\natural}
$$

Likewise, specializing Proposition 3.22 to the case of differential (rather than polydifferential) operators, we obtain

Proposition 4.13. Given $x \in \Gamma\left(\Lambda^{\bullet} A^{\vee}\right) \otimes_{R} \mathcal{U}(B)$ and $y \in \Gamma\left(\Lambda^{\bullet} L^{\vee}\right) \otimes_{R} \mathfrak{D}_{\mathrm{ver}}(B)$, we have

$$
\breve{\tau}_{\natural}(x)=y \quad \text { if and only if } \quad\left\{\begin{array}{l}
h_{\natural}(y)=0 \\
h_{\natural}(\llbracket Q, y \rrbracket)=0 \\
\sigma_{\natural}(y)=x
\end{array}\right.
$$

Both sides of (70) are universal enveloping algebras of dg Lie algebroids. Indeed, isomorphism (63) identifies $\Gamma\left(\Lambda^{\bullet} A^{\vee}\right) \otimes_{R} \mathcal{U}(B)$ with the universal enveloping algebra $\mathcal{U}(\mathcal{B})$ of the dg Lie algebroid $\mathcal{B} \rightarrow A[1]$, while $\Gamma\left(\Lambda^{\bullet} L^{\vee}\right) \otimes_{R} \mathfrak{D}_{\text {ver }}(B)$ is naturally identified with the universal enveloping algebra of the Fedosov dg Lie algebroid $\mathcal{F} \rightarrow \mathcal{M}$ (appearing in Proposition 2.14) since $\mathcal{F}$ is isomorphic to the pull back bundle $\mathrm{pr}^{*} T_{\mathrm{ver}} B$.

Following [40, Definition 2.6], we consider the associative algebra

$$
\mathcal{J}(B):=\operatorname{Hom}_{R}(\mathcal{U}(B), R)
$$

of $B$-jets on $M$ - the multiplication on $\mathcal{J}(B)$ arises as the map dual to the comultiplication on $\mathcal{U}(B)$. Dualizing the isomorphism of $R$-coalgebras

$$
\widetilde{\mathrm{pbw}}: \Gamma(S B) \stackrel{\cong}{\rightarrow} \mathcal{U}(B)
$$


appearing in Lemma 4.5, we obtain an isomorphism of associative $R$-algebras

$$
\widetilde{\mathrm{pbw}}^{\top}: \mathcal{J}(B) \stackrel{\cong}{\rightrightarrows} \Gamma\left(\hat{S}\left(B^{\vee}\right)\right) \text {. }
$$

The isomorphism $\widetilde{\mathrm{pbw}}^{\top}$ identifies the Grothendieck B-connection $\widetilde{\nabla}^{G}$ on $\mathcal{J}(B)$ introduced by NestTsygan [40, Proposition 2.7] and characterized by the relation

$$
\left\langle\widetilde{\nabla}_{b}^{G} \varphi \mid u\right\rangle=\varrho_{b}\langle\varphi \mid u\rangle-\langle\varphi \mid b \cdot u\rangle,
$$

for all $b \in \Gamma(B) ; \varphi \in \mathcal{J}(B)$; and $u \in \mathcal{U}(B)$, with the $B$-connection $\widetilde{\nabla}^{\xi}$ on $\Gamma\left(\hat{S}\left(B^{\vee}\right)\right)$ dual to the flat $B$-connection on $\Gamma(S B)$ defined by Equation (50). Indeed, the diagram

$$
\begin{array}{cc}
\mathcal{J}(B) \stackrel{\widetilde{\nabla}_{b}^{G}}{\longrightarrow} \mathcal{J}(B) \\
\widetilde{\mathrm{pbw}}^{\top} \downarrow \cong & \cong \widetilde{\mathrm{pbw}}^{\top} \\
\Gamma\left(\hat{S}\left(B^{\vee}\right)\right) \underset{\widetilde{\nabla}_{b}^{\natural}}{\longrightarrow} \Gamma\left(\hat{S}\left(B^{\vee}\right)\right)
\end{array}
$$

commutes for all $b \in \Gamma(B)$ since

$$
\begin{aligned}
\left\langle\widetilde{\mathrm{pbw}}^{\top}\left(\widetilde{\nabla}_{b}^{G} \varphi\right) \mid s\right\rangle & =\left\langle\widetilde{\nabla}_{b}^{G} \varphi \mid \widetilde{\mathrm{pbw}}(s)\right\rangle \\
& =\varrho_{b}\langle\varphi \mid \widetilde{\mathrm{pbw}}(s)\rangle-\langle\varphi \mid b \cdot \widetilde{\mathrm{pbw}}(s)\rangle \\
& =\varrho_{b}\langle\varphi \mid \widetilde{\mathrm{pbw}}(s)\rangle-\left\langle\varphi \mid \widetilde{\mathrm{pbw}}\left(\widetilde{\nabla}_{b}^{\xi} s\right)\right\rangle \\
& =\varrho_{b}\left\langle\widetilde{\mathrm{pbw}}^{\top}(\varphi) \mid s\right\rangle-\left\langle\widetilde{\mathrm{pbw}}^{\top}(\varphi) \mid \widetilde{\nabla}_{b}^{\xi} s\right\rangle \\
& =\left\langle\widetilde { \nabla } _ { b } ^ { \hat { \mathrm { pbw } } } \left(\widetilde{\mathrm{pb}}^{\top}(\varphi)|s\rangle\right.\right.
\end{aligned}
$$

for all $\varphi \in \mathcal{J}(B)$ and $s \in \Gamma(S B)$.

Given $x \in \mathcal{U}(B)$, we think of the multiplication $u \mapsto u \cdot x$ by $x$ from the right in $\mathcal{U}(B)$ as an endomorphism $\widetilde{R}_{x}$ of the $R$-module $\mathcal{U}(B)$, and we consider the dual endomorphism

$$
\widetilde{R}_{x}^{\top}: \mathcal{J}(B) \rightarrow \mathcal{J}(B) .
$$

For all $b \in \Gamma(B)$ and $x \in \mathcal{U}(B)$, we have

$$
\widetilde{\nabla}_{b}^{G} \circ \widetilde{R}_{x}^{\top}=\widetilde{R}_{x}^{\top} \circ \widetilde{\nabla}_{b}^{G},
$$

since

$$
\begin{aligned}
\left\langle\widetilde{\nabla}_{b}^{G} \circ \widetilde{R}_{x}^{\top}(\varphi) \mid u\right\rangle=\varrho_{b}\left\langle\widetilde{R}_{x}^{\top}(\varphi) \mid u\right\rangle-\left\langle\widetilde{R}_{x}^{\top}(\varphi) \mid b \cdot u\right\rangle=\varrho_{b}\langle\varphi \mid u \cdot x\rangle-\langle\varphi \mid b \cdot u \cdot x\rangle & \\
& =\left\langle\widetilde{\nabla}_{b}^{G} \varphi \mid u \cdot x\right\rangle=\left\langle\widetilde{\nabla}_{b}^{G} \varphi \mid \widetilde{R}_{x}(u)\right\rangle=\left\langle\widetilde{R}_{x}^{\top} \circ \widetilde{\nabla}_{b}^{G}(\varphi) \mid u\right\rangle
\end{aligned}
$$

for all $\varphi \in \mathcal{J}(B)$ and $u \in \mathcal{U}(B)$.

Lemma 4.14. For every $x \in \mathcal{U}(B)$, the endomorphism $\widetilde{R}_{x}^{\top}$ of $\mathcal{J}(B)$ is an R-linear differential operator on the algebra $\mathcal{J}(B)$. Furthermore, the map $x \mapsto \widetilde{R}_{x}^{\top}$ is a morphism of associative algebras from $\mathcal{U}(B)$ to the algebra of $R$-linear differential operators acting on the algebra $\mathcal{J}(B)$.

Proof. Adopting the Sweedler notation

$$
\Delta(u)=\sum_{(u)} u_{(1)} \otimes u_{(2)}=\sum_{(u)} u_{(2)} \otimes u_{(1)}
$$


to denote the cocommutative comultiplication on $\mathcal{U}(B)$ defined by Equation (17), and using the very definition of the multiplication in $\mathcal{U}(B)$ - see relations (16) or Equation (64) - we easily obtain that

$$
\widetilde{R}_{f}(u)=u \cdot f=\sum_{(u)} \varrho_{u_{(1)}}(f) \cdot u_{(2)}=\sum_{(u)} \varrho_{u_{(2)}}(f) \cdot u_{(1)}
$$

for all $f \in R \subset \mathcal{U}(B)$ and $u \in \mathcal{U}(B)$. Here the anchor map $\varrho: \Gamma(B) \rightarrow \mathfrak{X}(M)$ of the Lie algebroid $B \rightarrow M$ was implicitly extended to a morphism of associative algebras $\varrho: \mathcal{U}(B) \rightarrow \mathfrak{D}(M)$.

It follows that, for all $f \in C^{\infty}(M) \subset \mathcal{U}(B), \xi \in \mathcal{J}(B)$, and $u \in \mathcal{U}(B)$, we have

$$
\begin{aligned}
\left\langle\widetilde{R}_{f}^{\top}(\xi) \mid u\right\rangle & =\left\langle\xi \mid \widetilde{R}_{f}(u)\right\rangle=\left\langle\xi \mid \sum_{(u)} \varrho_{u_{(2)}}(f) \cdot u_{(1)}\right\rangle=\sum_{(u)}\left\langle\xi \mid u_{(1)}\right\rangle \cdot \varrho_{u_{(2)}}(f) \\
= & \sum_{(u)}\left\langle\xi \mid u_{(1)}\right\rangle \cdot \varrho_{u_{(2)} \cdot f}(1)=\sum_{(u)}\left\langle\xi \mid u_{(1)}\right\rangle \cdot\left\langle\mathbf{1} \mid u_{(2)} \cdot f\right\rangle=\sum_{(u)}\left\langle\xi \mid u_{(1)}\right\rangle \cdot\left\langle\widetilde{R}_{f}^{\top}(\mathbf{1}) \mid u_{(2)}\right\rangle \\
=\left\langle\xi \otimes \widetilde{R}_{f}^{\top}(\mathbf{1}) \mid \sum_{(u)} u_{(1)} \otimes u_{(2)}\right\rangle=\left\langle\xi \otimes \widetilde{R}_{f}^{\top}(\mathbf{1}) \mid \Delta(u)\right\rangle=\left\langle\Delta^{\top}\left(\xi \otimes \widetilde{R}_{f}^{\top}(\mathbf{1})\right) \mid u\right\rangle & =\left\langle\xi \cdot \widetilde{R}_{f}^{\top}(\mathbf{1}) \mid u\right\rangle,
\end{aligned}
$$

where the $B$-jet $\mathbf{1}$ is the morphism of left $R$-modules $\mathcal{U}(B) \ni u \mapsto \varrho_{u}(1) \in R$ associated with the constant function $1 \in R=C^{\infty}(M)$. Hence, we have

$$
\widetilde{R}_{f}^{\top}(\xi)=\xi \cdot \widetilde{R}_{f}^{\top}(\mathbf{1}), \quad \forall f \in C^{\infty}(M), \forall \xi \in \mathcal{J}(B),
$$

which shows that $\widetilde{R}_{f}^{\top}$ is indeed a differential operator of order zero on the algebra $\mathcal{J}(B)$.

For every $b \in \Gamma(B) \subset \mathcal{U}(B)$, the operator $\widetilde{R}_{b}$ is a coderivation of the left $R$-coalgebra $\mathcal{U}(B)$ and, consequently, $\widetilde{R}_{b}^{\top}$ is a derivation of the left $R$-algebra $\mathcal{J}(B)$.

For any two elements $u, v \in \mathcal{U}(B)$, we have $\widetilde{R}_{u v}^{\top}=\widetilde{R}_{u}^{\top} \circ \widetilde{R}_{v}^{\top}$ since $\widetilde{R}_{u v}=\widetilde{R}_{v} \circ \widetilde{R}_{u}$.

Since the universal enveloping algebra $\mathcal{U}(B)$ of the Lie algebroid $B \rightarrow M$ is generated multiplicatively by the elements of its subspace $C^{\infty}(M) \oplus \Gamma(B)$, it follows immediately that $\widetilde{R}_{x}^{\top}$ acts on the algebra of jets $\mathcal{J}(B)$ in the manner of a differential operator. The $R$-linearity of $\widetilde{R}_{x}^{\top}: \mathcal{J}(B) \rightarrow \mathcal{J}(B)$ is obvious.

Similarly, we can consider the Lie algebroid $\mathcal{B} \rightarrow A[1]$, the graded associative algebra of $\mathcal{B}$-jets on $A[1]$

$$
\mathcal{J}(\mathcal{B}):=\operatorname{Hom}_{\Gamma\left(\Lambda^{\bullet} A^{\vee}\right)}\left(\mathcal{U}(\mathcal{B}), \Gamma\left(\Lambda^{\bullet} A^{\vee}\right)\right),
$$

and the Grothendieck $\mathcal{B}$-connection $\nabla^{G}$ on $\mathcal{J}(\mathcal{B})$ characterized by the relation

$$
\left\langle\nabla_{b}^{G} \varphi \mid u\right\rangle=\bar{\varrho}_{b}\langle\varphi \mid u\rangle-\langle\varphi \mid b \cdot u\rangle,
$$

for all $b \in \Gamma(\mathcal{B}) ; \varphi \in \mathcal{J}(\mathcal{B})$; and $u \in \mathcal{U}(\mathcal{B})$.

It follows from the natural identification of the space of sections of the Lie algebroid $\mathcal{B} \rightarrow A[1]$ with $\Gamma\left(\Lambda^{\bullet} A^{\vee} \otimes B\right)$; the definition (46) of the anchor map $\bar{\varrho}$ of the Lie algebroid $\mathcal{B}$; and the isomorphism of graded associative algebras

$$
\mathcal{J}(\mathcal{B}) \cong \Gamma\left(\Lambda^{\bullet} A^{\vee}\right) \otimes_{R} \mathcal{J}(B)
$$

induced by the identification (63) that

$$
\nabla_{1 \otimes b}^{G}(\alpha \otimes \varphi)=\left(\nabla_{b}^{\mathrm{Bott}} \alpha\right) \otimes \varphi+\alpha \otimes\left(\widetilde{\nabla}_{b}^{G} \varphi\right)
$$

for all $b \in \Gamma(B) ; \alpha \in \Gamma\left(\Lambda A^{\vee}\right)$; and $\varphi \in \mathcal{J}(B)$. 
Given $x \in \mathcal{U}(\mathcal{B})$, we think of the multiplication $u \mapsto u \cdot x$ by $x$ from the right in $\mathcal{U}(\mathcal{B})$ as an endomorphism $R_{x}$ of the $\Gamma\left(\Lambda^{\bullet} A^{\vee}\right)$-module $\mathcal{U}(\mathcal{B}) \cong \Gamma\left(\Lambda^{\bullet} A^{\vee}\right) \otimes_{R} \mathcal{U}(B)$, and we consider the dual endomorphism

$$
R_{x}^{\top}: \Gamma\left(\Lambda^{\bullet} A^{\vee}\right) \otimes_{R} \mathcal{J}(B) \rightarrow \Gamma\left(\Lambda^{\bullet} A^{\vee}\right) \otimes_{R} \mathcal{J}(B) .
$$

The multiplication in $\mathcal{U}(\mathcal{B}) \cong \Gamma\left(\Lambda^{\bullet} A^{\vee}\right) \otimes_{R} \mathcal{U}(B)$ was defined in Equation (64).

For all $b \in \Gamma(\mathcal{B}) \cong \Gamma\left(\Lambda A^{\vee} \otimes B\right)$ and $x \in \mathcal{U}(\mathcal{B}) \cong \Gamma\left(\Lambda^{\bullet} A^{\vee}\right) \otimes_{R} \mathcal{U}(B)$, we have

$$
\nabla_{b}^{G} \circ R_{x}^{\top}=R_{x}^{\top} \circ \nabla_{b}^{G}
$$

since

$$
\begin{aligned}
\left\langle\nabla_{b}^{G} \circ R_{x}^{\top}(\varphi) \mid u\right\rangle=\bar{\varrho}_{b}\left\langle R_{x}^{\top}(\varphi) \mid u\right\rangle-\left\langle R_{x}^{\top}(\varphi) \mid b \cdot u\right\rangle=\bar{\varrho}_{b}\langle\varphi \mid u \cdot x\rangle-\langle\varphi \mid b \cdot u \cdot x\rangle & \\
& =\left\langle\nabla_{b}^{G} \varphi \mid u \cdot x\right\rangle=\left\langle\nabla_{b}^{G} \varphi \mid R_{x}(u)\right\rangle=\left\langle R_{x}^{\top} \circ \nabla_{b}^{G}(\varphi) \mid u\right\rangle
\end{aligned}
$$

for all $\varphi \in \mathcal{J}(\mathcal{B})$ and $u \in \mathcal{U}(\mathcal{B})$.

Lemma 4.15. For every $x \in \mathcal{U}(\mathcal{B})$, the endomorphism $R_{x}^{\top}$ of $\mathcal{J}(\mathcal{B})$ is a differential operator on the algebra $\mathcal{J}(\mathcal{B})$. Furthermore, the map $x \mapsto R_{x}^{\top}$ is a morphism of associative algebras from $\mathcal{U}(\mathcal{B})$ to the algebra of $\Gamma\left(\Lambda^{\bullet} A^{\vee}\right)$-linear differential operators acting on the algebra $\mathcal{J}(\mathcal{B})$.

The proof of Lemma 4.15 is similar to the proof of Lemma 4.14 and is therefore omitted.

The following lemma indicates that $\breve{\tau}_{\natural}(x)$ coincides with the operator $R_{x}^{\top}$ conjugated by the algebra isomorphism

$$
\mathrm{id} \otimes \widetilde{\mathrm{pbw}}^{\top}: \Gamma\left(\Lambda^{\bullet} A^{\vee}\right) \otimes_{R} \mathcal{J}(B) \rightarrow \Gamma\left(\Lambda^{\bullet} A^{\vee} \otimes \hat{S}\left(B^{\vee}\right)\right) .
$$

Lemma 4.16. For all $x \in \mathcal{U}(\mathcal{B}) \cong \Gamma\left(\Lambda^{\bullet} A^{\vee}\right) \otimes_{R} \mathcal{U}(B)$, the diagram

$$
\begin{array}{rr}
\Gamma\left(\Lambda^{\bullet} A^{\vee}\right) \otimes_{R} \mathcal{J}(B) & \stackrel{R_{x}^{\top}}{\longrightarrow} \Gamma\left(\Lambda^{\bullet} A^{\vee}\right) \otimes_{R} \mathcal{J}(B) \\
\operatorname{id} \otimes \widetilde{\mathrm{pbw}}^{\top} \mid \cong r & \cong \mid \operatorname{id} \otimes \widetilde{\mathrm{pbw}}^{\top} \\
\Gamma\left(\Lambda^{\bullet} A^{\vee} \otimes \hat{S}\left(B^{\vee}\right)\right) \underset{\breve{\tau}_{\sharp}(x)}{\longrightarrow} \Gamma\left(\Lambda^{\bullet} A^{\vee} \otimes \hat{S}\left(B^{\vee}\right)\right)
\end{array}
$$

commutes.

Proof. Given any element $x$ of $\Gamma\left(\Lambda^{\bullet} A^{\vee}\right) \otimes_{R} \mathcal{U}(B)$, let

$$
\mathcal{Y}_{x}=\left(\mathrm{id} \otimes \widetilde{\mathrm{pbw}}^{\top}\right) \circ R_{x}^{\top} \circ\left(\mathrm{id} \otimes \widetilde{\mathrm{pbw}}^{\top}\right)^{-1} .
$$

According to Lemma 4.15 , the operator $R_{x}^{\top}$ acts on the algebra $\mathcal{J}(\mathcal{B}) \cong \Gamma\left(\Lambda^{\bullet} A^{\vee}\right) \otimes_{R} \mathcal{J}(B)$ in the manner of a $\Gamma\left(\Lambda^{\bullet} A^{\vee}\right)$-linear differential operator and, consequently, the operator $\mathcal{Y}_{x}$ acts on the algebra $\Gamma\left(\Lambda^{\bullet} A^{\vee} \otimes \hat{S}\left(B^{\vee}\right)\right) \cong C^{\infty}(\mathcal{M})$ in the manner of a $\Gamma\left(\Lambda^{\bullet} A^{\vee}\right)$-linear differential operator. In other words, $\mathcal{Y}_{x}$ is a formal vertical differential operator on $\mathcal{B}$, i.e. an element of

$$
\Gamma\left(\Lambda A^{\vee} \otimes \hat{S}\left(B^{\vee}\right) \otimes S B\right) \cong \Gamma\left(\Lambda^{\bullet} A^{\vee}\right) \otimes_{R} \mathfrak{D}_{\text {ver }}(B) .
$$

According to Corollary 4.6, for all $b \in \Gamma(B)$ and $\alpha \otimes \varsigma \in \Gamma\left(\Lambda A^{\vee} \otimes \hat{S}\left(B^{\vee}\right)\right)$, we have

$$
\iota_{b} Q^{1,0}(\alpha \otimes \varsigma)=\nabla_{b}^{\text {Bott }} \alpha \otimes \varsigma+\alpha \otimes \widetilde{\nabla}_{b}^{\xi} \varsigma .
$$

By commutativity of diagram (72), we have

$$
\widetilde{\nabla}_{b}^{\xi} \varsigma=\widetilde{\mathrm{pbw}}^{\top} \circ \widetilde{\nabla}_{b}^{G} \circ\left(\widetilde{\mathrm{pbw}}^{\top}\right)^{-1}(\varsigma),
$$

and it then follows from Equation (73) that

$$
\iota_{b} Q^{1,0}=\left(\mathrm{id} \otimes \widetilde{\mathrm{pbw}}^{\top}\right) \circ \nabla_{1 \otimes b}^{G} \circ\left(\mathrm{id} \otimes \widetilde{\mathrm{pbw}}^{\top}\right)^{-1} .
$$


Equations (76), (75), and (74) yield

$$
\llbracket \iota_{b} Q^{1,0}, \mathcal{Y}_{x} \rrbracket=\left(\mathrm{id} \otimes \widetilde{\mathrm{pbw}}^{\top}\right) \circ \llbracket \nabla_{1 \otimes b}^{G}, R_{x}^{\top} \rrbracket \circ\left(\mathrm{id} \otimes \widetilde{\mathrm{pbw}}^{\top}\right)^{-1}=0
$$

for all $b \in \Gamma(B)$. Hence, we obtain

$$
\llbracket Q^{1,0}, \mathcal{Y}_{x} \rrbracket=0
$$

It is not difficult to check that the subspace

$$
\Gamma\left(\Lambda A^{\vee} \otimes \hat{S}\left(B^{\vee}\right) \otimes S B\right) \cong \Gamma\left(p^{\top}\left(\Lambda^{\bullet} A^{\vee}\right)\right) \otimes_{R} \mathfrak{D}_{\text {ver }}(B)
$$

of $\Gamma\left(\Lambda L^{\vee}\right) \otimes_{R} \mathfrak{D}_{\text {ver }}(B)$ is contained in the kernel of $h_{\natural}$ and is stable under $\llbracket d_{A}^{\text {Bott }}+X^{0,1},-\rrbracket$. Therefore, since

$$
Q=Q^{0,1}+Q^{1,0} ; \quad Q^{0,1}=d_{A}^{\text {Bott }}+X^{0,1} ; \quad \text { and } \quad \llbracket Q^{1,0}, \mathcal{Y}_{x} \rrbracket=0,
$$

we conclude that

$$
h_{\natural}\left(\mathcal{Y}_{x}\right)=0 \quad \text { and } \quad h_{\natural}\left(\llbracket Q, \mathcal{Y}_{x} \rrbracket\right)=h_{\natural}\left(\llbracket d_{A}^{\text {Bott }}+X^{0,1}, \mathcal{Y}_{x} \rrbracket\right)=0 .
$$

Let $\left\{\chi_{i}\right\}_{i=1, \ldots, r}$ and $\left\{\partial_{j}\right\}_{j=1, \ldots, r}$ be a pair dual local frames for the vector bundles $B^{\vee}$ and $B$ respectively. Then, with the usual multi-indices notations, $\left\{\chi^{I}\right\}_{I \in \mathbb{N}^{r}}$ and $\left\{\partial^{J}\right\}_{J \in \mathbb{N}^{r}}$ are the corresponding dual local frames for $\hat{S}\left(B^{\vee}\right)$ and $S(B)$ respectively. Locally, every formal vertical differential operator $\xi \in \Gamma\left(\Lambda A^{\vee} \otimes \hat{S}\left(B^{\vee}\right) \otimes S B\right)$ on the dg vector bundle $\mathcal{B} \rightarrow A[1]$ can be written as a linear combination $\xi=\sum_{I, J \in \mathbb{N}^{r}} \xi_{I, J} \otimes \chi^{I} \otimes \partial^{J}$ with coefficients $\xi_{I, J}=\left\langle\xi\left(\chi^{J}\right) \mid \partial^{I}\right\rangle$ in $\Gamma\left(\Lambda A^{\vee}\right)$. Furthermore, we have

$$
\sigma_{\natural}(\xi)=\sum_{J \in \mathbb{N}^{r}} \xi_{0, J} \otimes \widetilde{\operatorname{pbw}}\left(\partial^{J}\right) \quad \text { with } \quad \xi_{0, J}=\left\langle\xi\left(\chi^{J}\right) \mid 1\right\rangle .
$$

In particular, since

$$
\mathcal{Y}_{\alpha \otimes u}=\left(\mathrm{id} \otimes \widetilde{\mathrm{pbw}}^{\top}\right) \circ R_{\alpha \otimes u}^{\top} \circ\left(\mathrm{id} \otimes \widetilde{\mathrm{pbw}}^{\top}\right)^{-1}=\left(\left(\mathrm{id} \otimes \widetilde{\mathrm{pbw}}^{-1}\right) \circ R_{\alpha \otimes u} \circ\left(\mathrm{id} \otimes \widetilde{\mathrm{pbw}^{\prime}}\right)^{\top},\right.
$$

it follows that

$$
\begin{aligned}
\left\langle\mathcal{Y}_{\alpha \otimes u}\left(\chi^{J}\right) \mid 1\right\rangle= & \left\langle\chi^{J} \mid\left(\mathrm{id} \otimes \widetilde{\mathrm{pbw}}^{-1}\right) \circ R_{\alpha \otimes u} \circ(\mathrm{id} \otimes \widetilde{\mathrm{pbw}})(1)\right\rangle \\
& =\left\langle\chi^{J} \mid\left(\mathrm{id} \otimes \widetilde{\mathrm{pbw}}^{-1}\right) \circ R_{\alpha \otimes u}(1)\right\rangle=\alpha \cdot\left\langle\chi^{J} \mid \widetilde{\mathrm{pbw}}^{-1}(1 \cdot u)\right\rangle=\alpha \cdot\left\langle\chi^{J} \mid \widetilde{\mathrm{pbw}}^{-1}(u)\right\rangle
\end{aligned}
$$

and, according to Equation (77),

$$
\begin{array}{r}
\sigma_{\natural}\left(\mathcal{Y}_{\alpha \otimes u}\right)=\sum_{J \in \mathbb{N}^{r}}\left\langle\mathcal{Y}_{\alpha \otimes u}\left(\chi^{J}\right) \mid 1\right\rangle \otimes \widetilde{\mathrm{pbw}}\left(\partial^{J}\right)=\sum_{J \in \mathbb{N}^{r}} \alpha \cdot\left\langle\chi^{J} \mid \widetilde{\mathrm{pbw}}^{-1}(u)\right\rangle \otimes \widetilde{\mathrm{pbw}}\left(\partial^{J}\right) \\
=\alpha \otimes \widetilde{\mathrm{pbw}}\left(\sum_{J \in \mathbb{N}^{r}}\left\langle\chi^{J} \mid \widetilde{\mathrm{pbw}}^{-1}(u)\right\rangle \cdot \partial^{J}\right)=\alpha \otimes \widetilde{\mathrm{pbw}^{\prime}}\left(\widetilde{\mathrm{pbw}}^{-1}(u)\right)=\alpha \otimes u,
\end{array}
$$

for all $\alpha \otimes u \in \Gamma\left(\Lambda A^{\vee}\right) \otimes_{R} \mathcal{U}(B)$. Hence, we have $\sigma_{\natural}\left(\mathcal{Y}_{x}\right)=x$.

It follows from Proposition 4.13 that $\mathcal{Y}_{x}=\breve{\tau}_{\natural}(x)$.

The following proposition will play a key role in the proof of Theorem 4.12.

Proposition 4.17. In the contraction (70), the cochain map

$$
\breve{\tau}_{\mathrm{q}}: \Gamma\left(\Lambda^{\bullet} A^{\vee}\right) \otimes_{R} \mathcal{U}(B) \rightarrow \Gamma\left(\Lambda^{\bullet} L^{\vee}\right) \otimes_{R} \mathfrak{D}_{\mathrm{ver}}(B)
$$

respects the algebra and the coalgebra structures as well as the counit maps. Hence it realizes a morphism of dg Hopf algebroids from $\mathcal{U}(\mathcal{B})$ to $\mathcal{U}(\mathcal{F})$. 
Proof. It follows immediately from Lemma 4.16 that $\breve{\tau}_{\natural}$ is a morphism of algebras:

$$
\begin{aligned}
\breve{\tau}_{\natural}\left(x_{1} x_{2}\right)=\left(\mathrm{id} \otimes \widetilde{\mathrm{pbw}}^{\top}\right) \circ R_{x_{1} x_{2}}^{\top} \circ\left(\mathrm{id} \otimes \widetilde{\mathrm{pbw}}^{\top}\right)^{-1} \\
\quad=\left(\mathrm{id} \otimes \widetilde{\mathrm{pbw}}^{\top}\right) \circ R_{x_{1}}^{\top} \circ R_{x_{2}}^{\top} \circ\left(\mathrm{id} \otimes \widetilde{\mathrm{pbw}}^{\top}\right)^{-1}=\breve{\tau}_{\natural}\left(x_{1}\right) \cdot \breve{\tau}_{\natural}\left(x_{2}\right) .
\end{aligned}
$$

Proposition 3.23 established that $\breve{\tau}_{\natural}$ is a morphism of coalgebras. It is also clear that $\breve{\tau}_{\natural}$ respects the counit maps.

We are now ready to prove Theorem 4.12.

Proof of Theorem 4.12. Since $L=A \bowtie B$ is a matched pair, as vector spaces, tot $\left(\Gamma\left(\Lambda^{\bullet} A^{\vee}\right) \otimes_{R} \mathcal{D}_{\text {poly }}^{\diamond}\right)$ in Proposition 3.18 are isomorphic to tot $\left(\Gamma\left(\Lambda^{\bullet} A^{\vee}\right) \otimes_{R} \mathcal{U}(B)^{\diamond+1}\right)$. According to Proposition 3.18, the cochain maps $\breve{\tau}_{\natural}$ respects the cup products. Therefore, it suffices to prove that $\breve{\tau}_{\natural}$ in (41) respects the Lie algebra structures - the Lie bracket on tot $\left(\Gamma\left(\Lambda^{\bullet} A^{\vee}\right) \otimes_{R} \mathcal{U}(B)^{\diamond+1}\right)$ is the Gerstenhaber bracket of the $\mathrm{dg}$ Lie algebroid $\mathcal{B} \rightarrow A[1]$ as in Proposition 4.11. We know from the general theory of dg Lie algebroids - see Section 2.1 - that the Gerstenhaber bracket of a dg Lie algebroid is completely determined by its multiplication and comultiplication as shown by Equations (20) and (21). The conclusion thus follows immediately from Proposition 4.17.

\section{Appendix A. Semifull algebra contractions}

Let $\left(V, d_{V}\right)$ and $\left(W, d_{W}\right)$ be complexes: recall that a contraction of $\left(V, d_{V}\right)$ onto $\left(W, d_{W}\right)$ is the data of dg morphisms $\tau: W \rightarrow V, \sigma: V \rightarrow W$ and a contracting (degree minus one) homotopy $h: V \rightarrow V$ such that

$$
\sigma \tau=\mathrm{id}_{W}, \quad \tau \sigma-\mathrm{id}_{V}=d_{V} h+h d_{V}, \quad h \tau=0, \quad \sigma h=0, \quad h^{2}=0 .
$$

In the following well known homological perturbation lemma $[20,21]$ we assume that $V$ and $W$ are equipped with complete exhaustive decreasing filtrations $F^{\bullet} V$ and $F^{\bullet} W$ (we need this hypothesis to ensure convergence of the infinite sums in the following Lemma A.1), i.e.

$$
V=F^{0} V \supset F^{1} V \supset \cdots \supset F^{p} V \supset \cdots
$$

and the natural map $V \rightarrow \varliminf_{p} V / F^{p} V$ is an isomorphism, similarly for $W$. Furthermore, we assume that the differentials $d_{V}, d_{W}$ and the contraction data $(\tau, \sigma, h)$ preserve the filtrations. Recall that a perturbation of the differential $d_{V}$ on $V$ is a degree one map $\rho_{V}: V \rightarrow V$ such that $\left(d_{V}+\rho_{V}\right)^{2}=0$.

Lemma A.1 (Homological Perturbation). Given a perturbation $\rho_{V}: V \rightarrow V$ of the differential $d_{V}$ on $V$ such that $\rho_{V}\left(F^{p} V\right) \subset F^{p+1} V$, for all $p \geqslant 0$, the endomorphism $\rho_{W}$ of $W$ defined by

$$
\rho_{W}:=\sum_{l \geqslant 0} \sigma\left(\rho_{V} h\right)^{l} \rho_{V} \tau=\sum_{l \geqslant 0} \sigma \rho_{V}\left(h \rho_{V}\right)^{l} \tau
$$

is a perturbation of the differential $d_{W}$ on $W$, and the triple of maps

$$
\breve{\tau}:=\sum_{l \geqslant 0}\left(h \rho_{V}\right)^{l} \tau, \quad \breve{\sigma}:=\sum_{l \geqslant 0} \sigma\left(\rho_{V} h\right)^{l}, \quad \breve{h}:=\sum_{l \geqslant 0} h\left(\rho_{V} h\right)^{l}=\sum_{l \geqslant 0}\left(h \rho_{V}\right)^{l} h
$$

is a contraction of $\left(V, d_{V}+\rho_{V}\right)$ onto $\left(W, d_{W}+\rho_{W}\right)$.

In the following definition, taken from [42], we shall assume given two associative (but not necessarily graded commutative) products $\mu_{V}: V^{\otimes 2} \rightarrow V$ and $\mu_{W}: W^{\otimes 2} \rightarrow W$ : we do not require a priori $d_{V}$ and $d_{W}$ to be algebra derivations. 
Definition A.2. A contraction $(\tau, \sigma, h)$ of $\left(V, d_{V}\right)$ onto $\left(W, d_{W}\right)$ is a semifull algebra contraction if the following identities are satisfied

$$
\begin{aligned}
& h \mu_{V}(h \otimes h)=0, \quad h \mu_{V}(h \otimes \tau)=0, \quad h \mu_{V}(\tau \otimes h)=0, \quad h \mu_{V}(\tau \otimes \tau)=0, \\
& \sigma \mu_{V}(h \otimes h)=0, \quad \sigma \mu_{V}(h \otimes \tau)=0, \quad \sigma \mu_{V}(\tau \otimes h)=0, \quad \sigma \mu_{V}(\tau \otimes \tau)=\mu_{W} .
\end{aligned}
$$

Lemma A.3. Given (i) a pair of cochain complexes $\left(V, d_{V}\right)$ and $\left(W, d_{W}\right)$ carrying additional graded associative algebra structures; (ii) a semifull algebra contraction $(\tau, \sigma, h)$ of $\left(V, d_{V}\right)$ onto $\left(W, d_{W}\right)$; and (iii) a perturbation $\rho_{V}: V \rightarrow V$ of the differential $d_{V}$ satisfying $\rho_{V}\left(F^{p} V\right) \subset F^{p+1} V$ for all $p \geqslant 0$, the perturbed contraction $(\breve{\tau}, \breve{\sigma}, \breve{h})$ is a semifull algebra contraction of $\left(V, d_{V}+\rho_{V}\right)$ onto $\left(W, d_{W}+\rho_{W}\right)$.

Proof. Straightforward.

Lemma A.4. Given a semifull algebra contraction $(\tau, \sigma, h)$ of $\left(V, d_{V}\right)$ onto $\left(W, d_{W}\right)$, if $d_{V}$ is an algebra derivation, then $\tau: W \rightarrow V$ is a morphism of algebras.

Proof. Since $\sigma \mu_{V}(\tau \otimes \tau)=\mu_{W}$ and $h \mu_{V}(\tau \otimes \tau)=0$, we have

$$
\begin{aligned}
\mu_{V}(\tau \otimes \tau)=\left(\tau \sigma-d_{V} h-\right. & \left.h d_{V}\right) \mu_{V}(\tau \otimes \tau) \\
= & \tau \sigma \mu_{V}(\tau \otimes \tau)-d_{V} h \mu_{V}(\tau \otimes \tau)-h d_{V} \mu_{V}(\tau \otimes \tau) \\
= & \tau \mu_{W}-h \mu_{V}\left(d_{V} \otimes \operatorname{id}_{V}+\operatorname{id}_{V} \otimes d_{V}\right)(\tau \otimes \tau) \\
& =\tau \mu_{W}-h \mu_{V}(\tau \otimes \tau)\left(d_{W} \otimes i_{W}+\operatorname{id}_{W} \otimes d_{W}\right)=\tau \mu_{W}
\end{aligned}
$$

\section{REFERENCES}

[1] Ruggero Bandiera. Descent of Deligne-Getzler $\infty$-groupoids. 2017. arXiv: 1705.02880 [math.AT] .

[2] Ruggero Bandiera et al. "Shifted derived Poisson manifolds associated with Lie pairs". In: Comm. Math. Phys. 375.3 (2020), pp. 1717-1760. DOI: 10.1007/s00220-019-03457-w.

[3] Panagiotis Batakidis and Yannick Voglaire. "Atiyah classes and dg-Lie algebroids for matched pairs". In: J. Geom. Phys. 123 (2018), pp. 156-172. DOI: 10.1016/j.geomphys.2017.08.012.

[4] Kai Behrend and Ping Xu. "Differentiable stacks and gerbes". In: J. Symplectic Geom. 9.3 (2011), pp. 285341.

[5] Alexander Berglund. "Homological perturbation theory for algebras over operads". In: Algebr. Geom. Topol. 14.5 (2014), pp. 2511-2548. DOI: 10.2140/agt.2014.14.2511.

[6] Martin Bordemann et al. L-infinity Formality check for the Hochschild Complex of Certain Universal Enveloping Algebras. 2018. arXiv: 1807.03086 [math.QA].

[7] Raoul Bott. "Lectures on characteristic classes and foliations". In: Lectures on algebraic and differential topology (Second Latin American School in Math., Mexico City, 1971). Notes by Lawrence Conlon, with two appendices by J. Stasheff. Springer, Berlin, 1972, 1-94. Lecture Notes in Math., Vol. 279.

[8] Alberto S. Cattaneo and Giovanni Felder. "Relative formality theorem and quantisation of coisotropic submanifolds". In: Adv. Math. 208.2 (2007), pp. 521-548. DOI: 10.1016/j .aim.2006.03.010.

[9] Alberto S. Cattaneo, Giovanni Felder, and Lorenzo Tomassini. "Fedosov connections on jet bundles and deformation quantization". In: Deformation quantization (Strasbourg, 2001). Vol. 1. IRMA Lect. Math. Theor. Phys. de Gruyter, Berlin, 2002, pp. 191-202.

[10] Alberto S. Cattaneo, Giovanni Felder, and Lorenzo Tomassini. "From local to global deformation quantization of Poisson manifolds". In: Duke Math. J. 115.2 (2002), pp. 329-352. DOI: 10.1215/S0012-709402-11524-5.

[11] Zhuo Chen, Mathieu Stiénon, and Ping Xu. "From Atiyah classes to homotopy Leibniz algebras". In: Comm. Math. Phys. 341.1 (2016), pp. 309-349. DOI: 10.1007/s00220-015-2494-6.

[12] Zhuo Chen, Maosong Xiang, and Ping Xu. Hochschild cohomology of dg manifolds associated to integrable distributions. 2021. arXiv: 2103.08096 [math.DG].

[13] Vasiliy A. Dolgushev. "Covariant and equivariant formality theorems". In: Adv. Math. 191.1 (2005), pp. 147-177. DOI: $10.1016 / \mathrm{j}$.aim. 2004.02.001. 
[14] Vasily A. Dolgushev, Alexander E. Hoffnung, and Christopher L. Rogers. "What do homotopy algebras form?" In: Adv. Math. 274 (2015), pp. 562-605. DOI: 10.1016/j.aim.2015.01.014.

[15] Vasily A. Dolgushev and Christopher L. Rogers. "A version of the Goldman-Millson theorem for filtered $L_{\infty}$-algebras". In: J. Algebra 430 (2015), pp. 260-302. DOI: 10.1016/j .jalgebra.2015.01.032.

[16] Domenico Fiorenza and Marco Manetti. " $L_{\infty}$ structures on mapping cones". In: Algebra Number Theory 1.3 (2007), pp. 301-330.

[17] Kenji Fukaya. "Deformation theory, homological algebra and mirror symmetry". In: Geometry and physics of branes (Como, 2001). Ser. High Energy Phys. Cosmol. Gravit. IOP, Bristol, 2003, pp. 121-209.

[18] Murray Gerstenhaber. "On the deformation of rings and algebras". In: Ann. of Math. (2) 79 (1964), pp. 59-103.

[19] Murray Gerstenhaber. "The cohomology structure of an associative ring". In: Ann. of Math. (2) 78 (1963), pp. 267-288.

[20] V. K. A. M. Gugenheim, L. A. Lambe, and J. D. Stasheff. "Perturbation theory in differential homological algebra. II". In: Illinois J. Math. 35.3 (1991), pp. 357-373.

[21] Johannes Huebschmann and Tornike Kadeishvili. "Small models for chain algebras". In: Math. Z. 207.2 (1991), pp. 245-280.

[22] Johannes Huebschmann and James D. Stasheff. "Formal solution of the master equation via HPT and deformation theory". In: Forum Math. 14.6 (2002), pp. 847-868.

[23] Mikhail Kapranov. "Rozansky-Witten invariants via Atiyah classes". In: Compositio Math. 115.1 (1999), pp. 71-113. DOI: $10.1023 / \mathrm{A}: 1000664527238$.

[24] Hovhannes Khudaverdian and Theodore T. Voronov. "Higher Poisson brackets and differential forms". In: Geometric methods in physics. Vol. 1079. AIP Conf. Proc. Amer. Inst. Phys., Melville, NY, 2008, pp. 203-215.

[25] Maxim Kontsevich. "Deformation quantization of Poisson manifolds". In: Lett. Math. Phys. 66.3 (2003), pp. 157-216. DOI: 10.1023/B:MATH.0000027508.00421.bf.

[26] Y. Kosmann-Schwarzbach. "Graded Poisson brackets and field theory". In: Modern group theoretical methods in physics (Paris, 1995). Vol. 18. Math. Phys. Stud. Kluwer Acad. Publ., Dordrecht, 1995, pp. 189196.

[27] Alexei Kotov and Thomas Strobl. "Characteristic classes associated to Q-bundles". In: Int. J. Geom. Methods Mod. Phys. 12.1 (2015), pp. 1550006, 26. DOI: 10.1142/S0219887815500061.

[28] Camille Laurent-Gengoux, Mathieu Stiénon, and Ping Xu. "Exponential map and $L_{\infty}$ algebra associated to a Lie pair". In: C. R. Math. Acad. Sci. Paris 350.17-18 (2012), pp. 817-821. DOI: 10.1016/j .crma. 2012.08 .009$.

[29] Camille Laurent-Gengoux, Mathieu Stiénon, and Ping Xu. Poincaré-Birkhoff-Witt isomorphisms and Kapranov dg-manifolds. to appear in Adv. Math. 2014. arXiv: 1408.2903 [math.DG].

[30] Hsuan-Yi Liao, Mathieu Stiénon, and Ping Xu. "Formality and Kontsevich-Duflo type theorems for Lie pairs". In: Adv. Math. 352 (2019), pp. 406-482. DOI: 10.1016/j.aim.2019.04.047.

[31] Hsuan-Yi Liao, Mathieu Stiénon, and Ping Xu. "Formality theorem for g-manifolds". In: C. R. Math. Acad. Sci. Paris 355.5 (2017), pp. 582-589. DOI: 10.1016/j.crma.2017.03.008.

[32] Kirill C. H. Mackenzie. Double Lie algebroids and the double of a Lie bialgebroid. 1998. arXiv: math/ 9808081 [math.DG].

[33] Kirill C. H. Mackenzie. "Drinfel'd doubles and Ehresmann doubles for Lie algebroids and Lie bialgebroids". In: Electron. Res. Announc. Amer. Math. Soc. 4 (1998), pp. 74-87. DOI: 10.1090/S1079-6762-98-00050$\mathrm{X}$.

[34] Kirill C. H. Mackenzie. "Ehresmann doubles and Drinfel'd doubles for Lie algebroids and Lie bialgebroids". In: J. Reine Angew. Math. 658 (2011), pp. 193-245. DOI: 10.1515/CRELLE. 2011.092.

[35] Kirill C. H. Mackenzie and Tahar Mokri. "Locally vacant double Lie groupoids and the integration of matched pairs of Lie algebroids". In: Geom. Dedicata 77.3 (1999), pp. 317-330. DoI: 10.1023/A : 1005180403695.

[36] Rajan A. Mehta. "Q-algebroids and their cohomology". In: J. Symplectic Geom. 7.3 (2009), pp. 263-293.

[37] Rajan A. Mehta. Supergroupoids, double structures, and equivariant cohomology. Thesis (Ph.D.)University of California, Berkeley. ProQuest LLC, Ann Arbor, MI, 2006, p. 133. arXiv: math/0605356 [math.DG].

[38] Rajan A. Mehta, Mathieu Stiénon, and Ping Xu. "The Atiyah class of a dg-vector bundle". In: $C$. $R$. Math. Acad. Sci. Paris 353.4 (2015), pp. 357-362. DOI: 10.1016/j.crma.2015.01.019. 
[39] Tahar Mokri. "Matched pairs of Lie algebroids". In: Glasgow Math. J. 39.2 (1997), pp. 167-181. DOI: 10.1017/S0017089500032055.

[40] Ryszard Nest and Boris Tsygan. "Deformations of symplectic Lie algebroids, deformations of holomorphic symplectic structures, and index theorems". In: Asian J. Math. 5.4 (2001), pp. 599-635. DoI: 10.4310/ AJM. 2001.v5.n4.a2.

[41] Yong-Geun Oh and Jae-Suk Park. "Deformations of coisotropic submanifolds and strong homotopy Lie algebroids". In: Invent. Math. 161.2 (2005), pp. 287-360. DOI: 10.1007/s00222-004-0426-8.

[42] Pedro Real. "Homological perturbation theory and associativity". In: Homology Homotopy Appl. 2 (2000), pp. 51-88.

[43] Mathieu Stiénon, Luca Vitagliano, and Ping Xu. $A_{\infty}$ algebras from Lie pairs. Work in progress. 2021.

[44] Mathieu Stiénon and Ping Xu. "Atiyah classes and Kontsevich-Duflo type theorem for dg manifolds". In: Banach Center Publications 123 (2021), pp. 63-110. DOI: 10.4064/bc123-3.

[45] Mathieu Stiénon and Ping Xu. "Fedosov dg manifolds associated with Lie pairs". In: Math. Ann. 378.1-2 (2020), pp. 729-762. DOI: 10.1007/s00208-020-02012-6.

[46] Dmitry E. Tamarkin. Operadic proof of M. Kontsevich's formality theorem. Thesis (Ph.D.)-The Pennsylvania State University. ProQuest LLC, Ann Arbor, MI, 1999, p. 51.

[47] Arkady Yu. Vauntrob. "Lie algebroids and homological vector fields". In: Uspekhi Mat. Nauk 52.2(314) (1997), pp. 161-162. DOI: 10.1070/RM1997v052n02ABEH001802.

[48] Luca Vitagliano. "On the strong homotopy associative algebra of a foliation". In: Commun. Contemp. Math. 17.2 (2015), pp. 1450026, 34. DOI: 10.1142/S0219199714500266.

[49] Luca Vitagliano. "On the strong homotopy Lie-Rinehart algebra of a foliation". In: Commun. Contemp. Math. 16.6 (2014), pp. 1450007, 49. DOI: 10.1142/S0219199714500072.

[50] Luca Vitagliano. "Representations of homotopy Lie-Rinehart algebras". In: Math. Proc. Cambridge Philos. Soc. 158.1 (2015), pp. 155-191. DOI: 10.1017/S0305004114000541.

[51] Yannick Voglaire and Ping Xu. "Rozansky-Witten-type invariants from symplectic Lie pairs". In: Comm. Math. Phys. 336.1 (2015), pp. 217-241. DOI: 10.1007/s00220-014-2221-8.

[52] Theodore Th. Voronov. "Q-manifolds and Mackenzie theory". In: Comm. Math. Phys. 315.2 (2012), pp. 279-310. DOI: $10.1007 / \mathrm{s} 00220-012-1568-\mathrm{y}$.

[53] Thomas Willwacher. "The homotopy braces formality morphism". In: Duke Math. J. 165.10 (2016), pp. 1815-1964. DOI: 10.1215/00127094-3450644.

[54] Ping Xu. "Gerstenhaber algebras and BV-algebras in Poisson geometry". In: Comm. Math. Phys. 200.3 (1999), pp. 545-560. DOI: $10.1007 / \mathrm{s} 002200050540$.

[55] Ping Xu. "Quantum groupoids". In: Comm. Math. Phys. 216.3 (2001), pp. 539-581. DoI: 10 . 1007 / s002200000334.

Dipartimento di Matematica, Sapienza Università di Roma

Email address: bandiera@mat.uniroma1.it

Department of Mathematics, Pennsylvania State University

Email address: stienon@psu.edu

Department of Mathematics, Pennsylvania State University

Email address: ping@math.psu.edu 